\title{
Application of Artificial Intelligence in Gas Turbine Control and Modelling
}

by

\author{
Roni Daher \\ A thesis submitted to \\ the Faculty of Graduate Studies and Research \\ in partial fulfillment of \\ the requirements for the degree of \\ Master of Applied Science \\ Aerospace \\ Ottawa-Carleton Institute for \\ Mechanical and Aerospace Engineering \\ Department of \\ Mechanical and Aerospace Engineering \\ Carleton University \\ Ottawa, Ontario \\ Canada \\ August $30^{\text {th }}, 2005$ \\ (C) Copyright \\ 2005 - Roni Daher
}




$\begin{array}{ll}\begin{array}{l}\text { Library and } \\ \text { Archives Canada }\end{array} & \begin{array}{l}\text { Bibliothèque et } \\ \text { Archives Canada }\end{array} \\ \begin{array}{l}\text { Published Heritage } \\ \text { Branch }\end{array} & \begin{array}{l}\text { Direction du } \\ \text { Patrimoine de l'édition }\end{array} \\ \begin{array}{l}\text { 395 Wellington Street } \\ \text { Ottawa ON K1A ON4 }\end{array} & \begin{array}{l}\text { 395, rue Wellington } \\ \text { Ottawa ON K1A ON4 } \\ \text { Canada }\end{array}\end{array}$

Your file Votre référence

ISBN: 0-494-10085-0

Ourfile Notre référence

ISBN: 0-494-10085-0

NOTICE:

The author has granted a nonexclusive license allowing Library and Archives Canada to reproduce, publish, archive, preserve, conserve, communicate to the public by telecommunication or on the Internet, loan, distribute and sell theses worldwide, for commercial or noncommercial purposes, in microform, paper, electronic and/or any other formats.

The author retains copyright ownership and moral rights in this thesis. Neither the thesis nor substantial extracts from it may be printed or otherwise reproduced without the author's permission.
AVIS:

L'auteur a accordé une licence non exclusive permettant à la Bibliothèque et Archives Canada de reproduire, publier, archiver, sauvegarder, conserver, transmettre au public par télécommunication ou par l'Internet, prêter, distribuer et vendre des thèses partout dans le monde, à des fins commerciales ou autres, sur support microforme, papier, électronique et/ou autres formats.

L'auteur conserve la propriété du droit d'auteur et des droits moraux qui protège cette thèse. $\mathrm{Ni}$ la thèse ni des extraits substantiels de celle-ci ne doivent être imprimés ou autrement reproduits sans son autorisation.
In compliance with the Canadian

Privacy Act some supporting forms may have been removed from this thesis.

While these forms may be included in the document page count, their removal does not represent any loss of content from the thesis.
Conformément à la loi canadienne sur la protection de la vie privée, quelques formulaires secondaires ont été enlevés de cette thèse.

Bien que ces formulaires aient inclus dans la pagination, il n'y aura aucun contenu manquant.

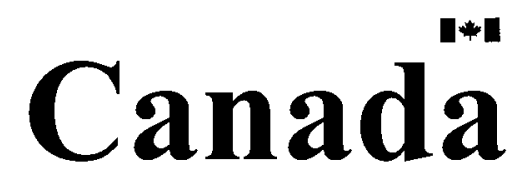


The undersigned recommend to

the Faculty of Graduate Studies and Research

acceptance of the thesis

\section{Application of Artificial Intelligence in Gas Turbine Control and Modelling}

submitted by

Roni Daher

in partial fulfillment of the requirements for the degree of Master of Applied Science

Chairman, Dr. J.C. Beddoes

Department of Mechanical and Aerospace Engineering

Thesis Co-Supervisor, Dr. J.E.D. Gauthier

Thesis Co-Supervisor, Dr. A. Jnifene

Carleton University

August 2005 


\section{Abstract}

The subject of gas turbine modelling and control using expert knowledge systems is discussed in this thesis. Modelling of nonlinear dynamic system using feedforward artificial neural networks using data generated by a simulated gas turbine program is introduced. Two artificial neural networks are used, depicting the nonlinear relationship between gas generator speed and fuel flow, and turbine inlet temperature and fuel flow, respectively. Training, validation, and test data are used to evaluate the generalization ability of these artificial neural networks.

A fuzzy logic control approach is applied to gas turbine fuel schedules and a controller for a gas turbine model is subsequently designed. The linguistic fuzzy rules and membership functions are presented, and the response of the gas turbine to the fuzzy logic controller and a PID controller action is evaluated. Overall, the fuzzy logic controller showed comparable results with the PID controller. 


\section{Table of Contents}

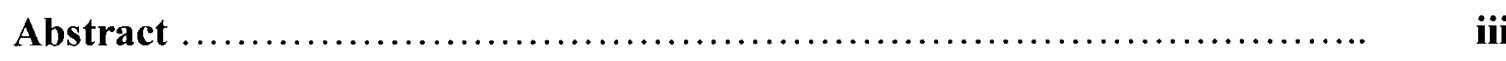

Table of Contents .......................................................... iv

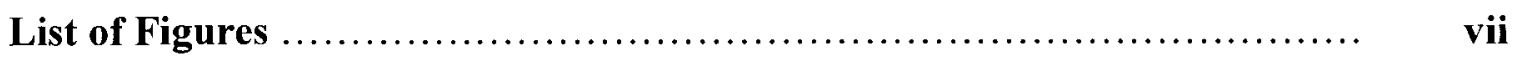

List of Tables ............................................................

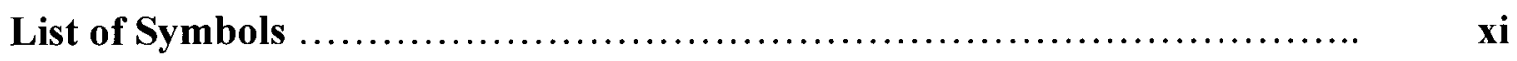

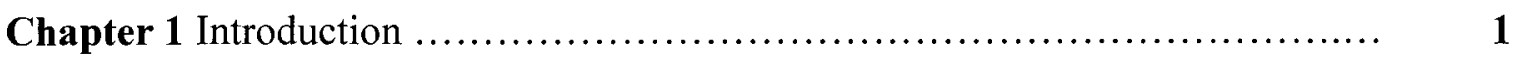

1.1 History of gas turbine engines ........................................ 3

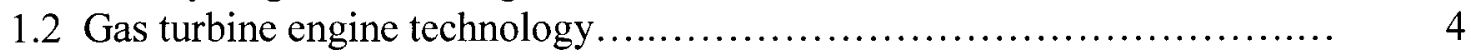

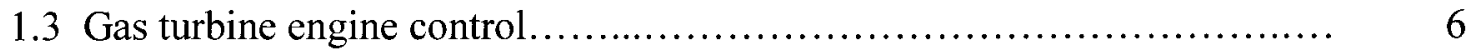

1.3.1 Propulsion control background................................ 7

1.3.2 History of GTE control technologies.............................. 10

1.3.3 Control methodology ......................................... 11

1.3.3.1 Time-domain based methodology ............................ 11

1.3.3.2 Expert knowledge system methodology ..................... 14

1.4 Thesis presentation.................................................... 16

Chapter 2 Literature review .............................................. 18

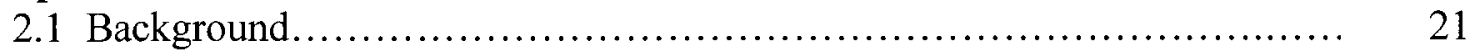

2.1.1 Gas turbine engine control function.............................. 22

2.1.2 Transient performance...................................... 23

2.1.3 Acceleration and deceleration schedules.......................... 24

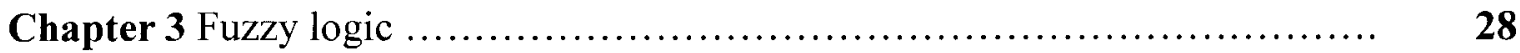

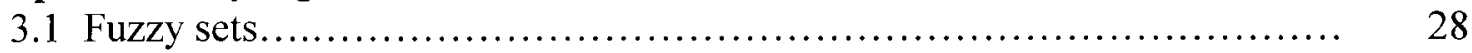

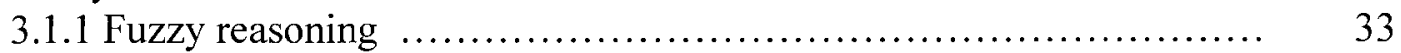

3.1.2 Sugeno fuzzy model.......................................... 39

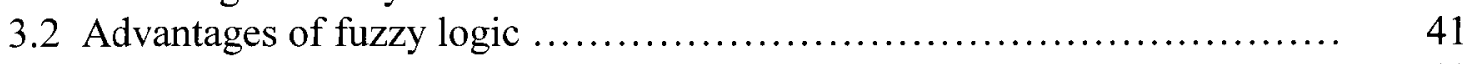

3.3 Disadvantages of fuzzy logic ....................................... 41

Chapter 4 Artificial neural networks ..................................... 43

4.1 Introduction to artificial neural networks .............................. 43

4.1.1 Evolution of artificial neural networks............................. 44

4.2 Brief description of neurons......................................... 46

4.3 Artificial neural networks......................................... 49

4.3.1 Architecture of ANNs......................................... 51 
4.3.2 Training of ANNs ............................................. 52

4.3.3 ANNs learning algorithm ..................................... 53

4.3.3.1 Backpropagation method ............................... 54

4.3.3.2 Levenberg - Marquardt training method ................... 56

4.4 Types of artificial neural networks .................................... 57

4.4.1 Multilayer perceptron ........................................ 57

4.4.2 Feedforward artificial neural networks........................... 59

4.5 Advantages of artificial neural networks .............................. 61

4.6 Disadvantages of artificial neural networks .............................. 62

4.7 Modelling with artificial neural networks ............................... 62

Chapter 5 Simulation analysis ........................................... 64

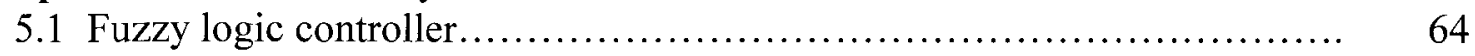

5.1.1 Performance characteristics of GTE ........................... 65

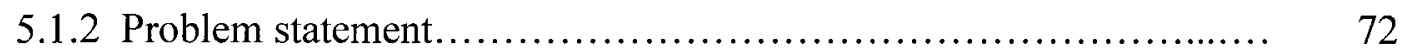

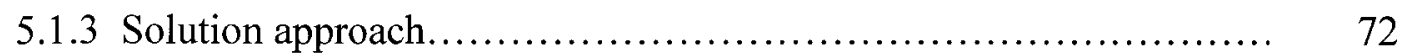

5.2 Fuzzy logic control design ........................................ 77

5.2.1 Define inputs and outputs for the control scheme .................... 77

5.2.2 Fuzzy system process ......................................... 78

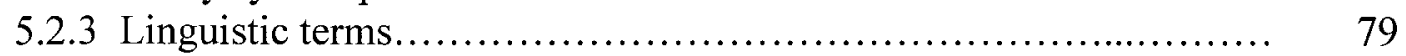

5.2 .4 Antecedent-consequent propositions ............................. 80

5.2 .5 Fuzzy membership functions .................................. 81

5.2.6 Mamdani system application to FLC acceleration schedule ........... $\quad 82$

5.2.7 Sugeno fuzzy inference applied to the deceleration schedule ......... 84

5.3 Application of fuzzy control to the thermodynamic model ................. 87

5.3.1 Fuzzy control strategy .............................................. 88

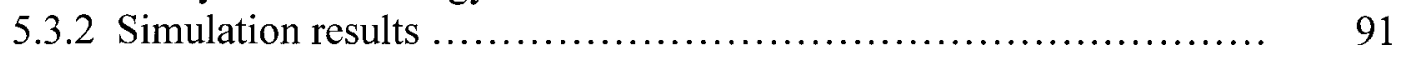

5.4 Dynamic system modelling using ANNs............................... 94

5.4 .1 Design procedure............................................ 95

5.4.2 Data collection.................................................. 97

5.4 .3 Neural process modelling.................................. 97

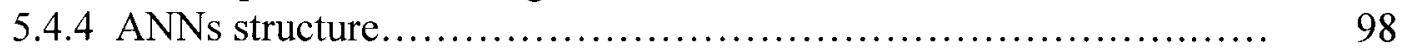

5.5 Results............................................................. 100

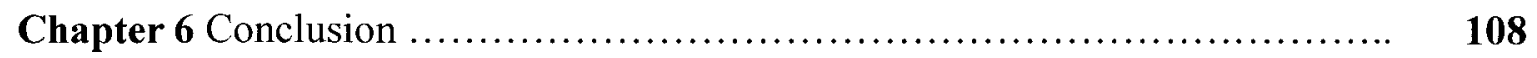

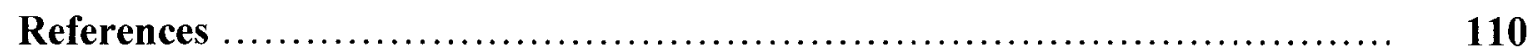

Appendix A Fuzzy Logic Example (Mamdani -type inference engine)............ A-1

Fuzzy logic example with two inputs - one output variables................. A-2

System design ......................................................... A-

Appendix B GasTurb Data for Fuel Schedule............................... B-1

GasTurb data for acceleration and deceleration schedules of a 1 MW GTE. B-2 
GasTurb data for fuzzy logic controller (fuel schedules worksheet).

Thermodynamic design variables.

Surge fuel flow ratio.

Fuel schedules (acceleration \& deceleration schedules)

Appendix C Simulink and Matlab Codes for 1 MW GTE Fuel Schedules

Acceleration deceleration Simulink model.

Sugeno deceleration fuel schedule.

Mamdani acceleration fuel schedule.

Appendix D Zero-Emission GT Model

Description of the Zero-Emission GT model......................... D-2

Sugeno FLC for $60 \mathrm{~kW}$ gas turbine Matlab codes....................... D-5

Modified membership functions FLC for $60 \mathrm{~kW}$ GTE ................... D-6

Appendix E Artificial Neural Networks Codes............................. E-1

ANN for the TIT versus fuel flow and $\mathrm{N}_{\mathrm{GG}}$ versus fuel flow of a $1 \mathrm{MW}$

GTE (at transient performance).

E-2

Artificial neural networks Matlab code

E-4 


\section{List of Figures}

Figure 1-1: The M88-2 gas turbine engine ............................ 5

Figure 1-2: Propulsion efficiency characteristics of aero-engines .............. 5

Figure 1-3: Diagram of simple engine control system...................... 8

Figure 1-4: Gas turbine fuel control system operating boundaries............. 9

Figure 2-1: Typical compressor map.................................. 26

Figure 3-1: Membership function for crisp and fuzzy subsets ................ 29

Figure 3-2: Typical membership functions used in fuzzy logic control ......... 31

Figure 3-3: Flowchart of fuzzy logic controller........................ 33

Figure 3-4: Fuzzy input variable................................... 34

Figure 3-5: Rule calculation for a two-input single-output Mamdani FIS.

NL (negative large) and NM (negative medium) are fuzzy linguist terms..................................................... 38

Figure 3-6: Linear approximation using Sugeno fuzzy model................ 40

Figure 4-1: Biological neuron ......................................... 45

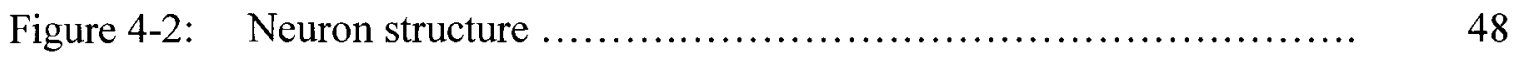

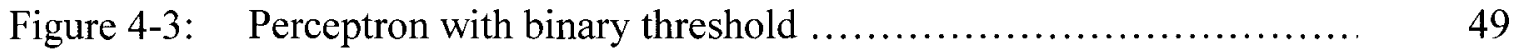

Figure 4-4: Feedforward non-recurrent ANN ........................... 50

Figure 4-5: Recurrent artificial neural network topology $\quad$................... 51

Figure 4-6: Two-layer ANN with sigmoid and linear activation functions...... 52

Figure 4-7: Backpropagation learning algorithm......................... 54

Figure 4-8: $\quad$ Sigmoid activation functions............................... 59

Figure 4-9: Tanh activation function .................................. 59

Figure 4-10: Two-layer feedforward artificial neural network................. 61

Figure 5-1: Schematic of a generic compressor map with DP shown........... 66

Figure 5-2: Transient acceleration schedule on a compressor map............. 70

Figure 5-3: Schematic of compressor map with AS and DS shown............ 71 
Figure 5-4: Schematic of acceleration and deceleration schedules

Figure 5-5: Compressor map with surge, operating, and deceleration schedule lines shown.

Figure 5-6: Fuel schedule on a fuel flow ratio versus gas generator speed $\left(\mathrm{N}_{\mathrm{GG}}\right)$ graph........................................... 76

Figure 5-7: Linguistic variable $\mathrm{N}_{\mathrm{GG}}$ with five linguistic terms.............. 80

Figure 5-8: Membership functions for $\mathrm{N}_{\mathrm{GG}}$ input vector................... 82

Figure 5-9: Membership functions for fuel ratio output vector.............. 82

Figure 5-10: Fuzzy acceleration response versus GasTurb response............. 84

Figure 5-11: Input vector $N_{G G}$ membership functions; deceleration............ 86

Figure 5-12: Output membership functions (fuel ratio unit); deceleration........ 86

Figure 5-13: Fuzzy deceleration response versus GasTurb response ............ 87

Figure 5-14: Errorspeed input membership function........................ 88

Figure 5-15: Errordot input membership function........................ 89

Figure 5-16: Fuel output membership functions (Zero-Emission GT)........... 89

Figure 5-17: PID controller response ................................ 92

Figure 5-18: Response of fuzzy logic controller ......................... 93

Figure 5-19: Feedforward ANN with one hidden layer and 9 hidden neurons; the input is $\mathrm{N}_{\mathrm{GG}}$ or TIT and the output is fuel flow............... 99

Figure 5-20: NN compared to GasTurb simulation model training data $\left(\mathrm{N}_{\mathrm{GG}}\right) \ldots \quad 100$

Figure 5-21: $\mathrm{N}_{\mathrm{GG}}$ validation data distribution........................... 101

Figure 5-22: $\mathrm{N}_{\mathrm{GG}}$ validation error................................. 102

Figure 5-23: $\mathrm{N}_{\mathrm{GG}}$ test error...................................... 102

Figure 5-24: NN compared to GasTurb TIT training data variance............. 103

Figure 5-25: TIT validation data....................................... 104

Figure 5-26: TIT validation data error.................................... 105

Figure 5-27: TIT test data variance................................... 105

Figure 5-28: TIT test data error....................................... 106 
Figure A-1: The degree of membership functions for the two inputs: TIT and

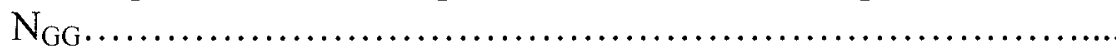

Figure A-2: The membership functions of output variable: Wf .............. A-3

Figure A-3: The rule-base represented in a matrix form.................... A-4

Figure A-4: Inputs representation................................... A-5

Figure A-5: Rule firing strength; (a) for Rule 2 it is 0.6 and (b) for Rulel it is 0.3

Figure A-6: Fuzzy inference system with centroid of area method; (a) Rule 2 crisp output calculation and (b) Rule 1 crisp output, both outputs are 3 .

Figure C-1: Fuzzy fuel schedules for a 1 MW GTE: (a) Mamdani acceleration schedule, (b) Sugeno deceleration schedule......................

Figure D-1: Zero-Emission GT Simulink model.............................

Figure D-2: Fuzzy control configuration of Zero-Emission GT............. D-4

Figure D-3: FLC Errorspeed input modified membership functions........... D-7

Figure D-4: FLC Errordot input modified membership functions............. D-7

Figure E-1: ANN Simulink model for a $1 \mathrm{MW}$ GTE; (a) $\mathrm{N}_{\mathrm{GG}}$ ANN, (b) TIT ANN.... 


\section{List of Tables}

Table 5-1: GasTurb parameters for $1 \mathrm{MW}$ twin-shaft turboshaft engine........... 73

Table 5-2: Rule-base for fuel flow membership functions; acceleration schedule (Mamdani fuzzy inference system).................... $\quad 80$

Table 5-3: Fuzzy output control value (Acceleration schedule)................ 81

Table 5-4: Rule-base for deceleration fuel schedule......................... 84

Table 5-5: Fuzzy output control value (Deceleration schedule)................ 85

Table 5-6: Fuel flow output control values (Zero-Emission GT) ................. 90

Table 5-7: Rule-base for fuel change (sugeno)............................ 90

Table 5-8: Training and test error..................................... 106 


\section{List of Symbols}

\begin{tabular}{|c|c|}
\hline$a$ & Actual output vector after iteration in ANN \\
\hline A $_{\text {. }}$ & Crisp set \\
\hline$\tilde{\mathrm{A}}, \check{Z}$ & Fuzzy sets \\
\hline $\mathrm{ANN}$ & Artificial neural network (also called NN) \\
\hline$b$ & Bias of neural network \\
\hline$C$ & Centroid (Mamdani inference system) \\
\hline e & Error in training of ANN \\
\hline e & Error in Levenberg-Marquardt training method (ANN) \\
\hline EPR & Engine pressure ratio \\
\hline$f()$ & Activation function in ANN \\
\hline FADEC & Full-authority digital engine controller \\
\hline FIS & Fuzzy inference system \\
\hline FLC & Fuzzy logic controller \\
\hline $\mathrm{g}$ & Syntactic rule \\
\hline GA & Genetic algorithm \\
\hline GTE & Gas turbine engine \\
\hline HP & High pressure \\
\hline $\mathrm{I} / \mathrm{O}$ & input - output \\
\hline INA & Inverse Nyquist Array \\
\hline $\mathbf{J}$ & Jacobian matrix \\
\hline$K$ & Number of rule for Sugeno inference system \\
\hline $\mathrm{L}$ & Linguistic term \\
\hline LQR & Linear Quadratic Regulator \\
\hline$m$ & Number of propositions \\
\hline$M$ & Rule-base size \\
\hline$M_{o}$ & Mach number \\
\hline $\mathrm{N}$ & Engine speed or Negative in fuzzy logic \\
\hline $\mathrm{N}_{\mathrm{GG}}$ & Gas generator speed or gas generator spool speed \\
\hline$n$ & Number of sample data \\
\hline NASA & National Aeronautics and Space Administration \\
\hline NB & Negative big in FLC \\
\hline NM & Negative medium in FLC \\
\hline $\mathrm{NN}$ & Neural network in FLC \\
\hline $\mathrm{NO}_{x}$ & Nitric oxide \\
\hline NS & Negative small in FLC \\
\hline$p$ & Input vector in $\mathrm{ANN}$ \\
\hline $\mathrm{P}$ & Positive in FLC \\
\hline$P_{1}$ & Ambiant pressure \\
\hline $\mathrm{P}_{3}$ & Compressor exit static pressure \\
\hline
\end{tabular}




$\begin{array}{ll}\text { PB } & \text { Positive big in FLC } \\ \text { PID } & \text { Proportional integral derivative } \\ \text { PM } & \text { Positive medium in FLC } \\ \text { PS } & \text { Positive small in FLC } \\ \text { PW } & \text { Pratt and Whitney } \\ Q & \text { Number of hidden neurons } \\ t & \text { Target vector in ANN } \\ \mathrm{T}_{1} & \text { Ambiant temperature } \\ \text { TIT } & \text { Turbine inlet temperature } \\ \mathrm{u} & \text { Variable } \\ \mathrm{v} & \text { Variable } \\ w & \text { Weight of neural network neurons } \\ W & \text { Sum of network weights } \\ \mathrm{Wf} & \text { Fuel flow } \\ \mathrm{Wf} / \mathrm{P} 3 & \text { Fuel ratio } \\ \mathrm{X} & \text { Universe of discourse } \\ \mathrm{z} & \text { Fuzzy implication function } \\ \text { Zero } & \text { Zero in FLC } \\ \Gamma(\text { ) } & \text { Vector-value function in ANN } \\ \zeta(x, y) & \text { Consequent function (Sugeno) } \\ \mu() & \text { Membership function (Fuzzy logic) }\end{array}$

\section{Greek Symbols}

$\begin{array}{ll}\Delta & \text { Difference sign } \\ \sum & \text { Summation sign } \\ \gamma & \text { Learning rate (backpropagation method in ANN) } \\ \delta_{I} & \text { Pressure correction factor } \\ \theta & \text { Scalar in ANN } \\ \theta_{1} & \text { Temperature correction factor } \\ \lambda & \text { Rule firing strength in FLC } \\ \chi & \text { Variable, real number (fuzzy logic) } \\ \psi & \text { Number of random sets }\end{array}$

\section{Subscripts}

$\begin{array}{ll}\text { GG } & \text { Gas generator } \\ \text { ref } & \text { Reference } \\ \text { S-shaped } & \text { Activation function } \\ \text { tanh } & \text { Tanh function (activation function) } \\ \check{Z} & \text { Single output fuzzy set }\end{array}$




\section{Chapter 1}

\section{Introduction}

In engineering, artificial intelligence includes fuzzy logic and artificial neural network techniques. These expert knowledge systems are applied in control, modelling, and feature recognition problems.

This thesis represents the results of a fuzzy logic controller design for a gas turbine engine (GTE) under development. It represents an approach for modelling the transient performance of a GTE using an artificial neural network. The work is motivated by the complexity in modelling and controlling gas turbine engines due to the nonlinear nature of their behaviour. The problem of nonlinearity can be regarded as a root cause for control instability, the loss of smooth transition between operating points and reduced efficiency in the performance of the GTE at off-design points. In the absence of a precise mathematical model, in GTEs under development, too many critical parameters like the gas generator (GG) spool speed, turbine inlet temperature, pressure ratio, rotor inertia, etc., are under-defined. Such anomalies are expected to be overcome by the introduction of control technologies based on expert knowledge systems, mainly the fuzzy logic control (FLC). These rule-base systems can provide basic advantages for GTE control. The potential advantages of FLC are the ease of implementation, an increase in the smooth transition between operating points and a reduction in the peak operation temperatures. Ensuring smooth transition between operating points can be done in real-time over the entire envelope of the GTE operation, while staying within the safe 
operation limits represented by the minimum and maximum fuel flow to avoid combustor flameouts, compressor surge or stall and excessive turbine temperature or speed. Furthermore, the reduction of peak temperature can decrease the thermal stresses resulting from overshoots during the startup or during swift acceleration while ensuring the stability of the machinery in question.

FLC applications can be widely employed in such fields where an accurate mathematical model of any process subject to control cannot be developed. In such situations, the control system based on expert knowledge is configured and expressed in intuitive terms. The rule-base control laws can be developed and modified allowing quantification of the controller inputs. The control parameters are then established in a quantified way that allows the developer to overcome the imprecision in the process modelling. The inherent nonlinearity in the gas turbine engine discussed earlier is an example of such a condition. The FLC permits the designer to meet the desired design conditions despite vague assumptions.

The proposed controller in this thesis is developed to meet the design requirements of a $1 \mathrm{MW}$ twin shaft GTE for marine applications. The performance of the proposed controller is assessed by a simulation created using MatLab/Simulink software. Furthermore, the simulated GTE transient performance is modelled using feedforward artificial neural networks. The relationships between the gas generator spool speed and the fuel flow, along with those between the turbine inlet temperature (TIT) and the fuel flow, are regenerated using two-layer artificial neural networks. 


\subsection{History of gas turbine engines}

Gas turbine engine (GTE) control systems have developed for the purpose of meeting particular needs or to comply with predetermined operating conditions and strict environmental requirements; e.g., NOx emissions standards. It would be interesting to review the development of such systems from their very early simple lever and throttle positioning, to the more complex multivariable and dual-channel full-authority digital engine controller (FADEC). But before the subject of GTE control modes is tackled, a brief introduction to the gas turbine engine and its development will be made.

Gas turbine engines were the subject of investigation since ancient history. In 150 B.C.E., a Greek philosopher and mathematician, Hero, invented the Aelopile that uses the energy of the rising hot air as rotating power. Some 1650 years later, in 1500, Leonardo da Vinci portrayed a rotating boiling spit driven by hot flue gases from a chimney. But it was not until 1872 that the first authentic gas turbine engine with a multistage turbine section and a compressor was introduced by Dr. F. Stolze. This model was based on Isaac Newton's theoretical approach to the three laws of motion. Ever since, the theory and applications of gas turbines have drawn more interest, and in 1918, Dr. Stanford A. Moss developed the GE Turbo supercharger engine paving the way in 1930 for Sir Frank Whittle's design of a jet propulsion gas turbine. The engine was produced and tested in April 1937. Nine years later, the Ernest Heinkel Aircraft company developed and successfully flight-tested its first gas turbine jet, the HE178, and in 1941 Whittle designed the first turbojet airplane, the Gloster Meteor (Berton, 2004). 
A brief presentation of the technology of gas turbines will be included in the following sections.

\subsection{Gas turbine engine technology}

A typical gas turbine engine consists of a compressor, a combustion chamber and a turbine (Figure 1-1). The design of gas turbine engines emphasizes fast response times and high spool speeds. In aircraft with high-thrust engines, for example, the acceleration time from idle to maximum take-off power is achieved in less then 60 seconds (Giampaolo, 1997). The thermodynamic principles governing the theory behind gas turbine cycles, i.e., the Brayton cycle and its derivatives, are drawn from the laws of physics. In terms of energy utilization, there are still distinct types of gas turbines categorized by different design characteristics such as the number of spools (single- or twin-spool, which is the general configuration for many modern gas turbines, namely the Eurojet EJ200, the SNECMA M88, the GE F404/RM12, F110/400), bypass ratio, principle of compression, and regeneration of exhaust gas energy. In brief, four major classes of gas turbine engine are described in the literature: turbojet, turbofan, turboprop, and turboshaft (Huenecke, 1997). These engines all have the basic common arrangement of a compressor in which work is converted into kinetic energy, a combustor (combustion chamber where fuel is injected and thermal power is generated) and a turbine section (power turbine or free turbine) in which the excess power (not needed to drive the compressor) is converted to thrust and also real work in power generators. Other configurations comprise an inlet and a nozzle with an afterburner. The performance of

these engines is beyond the scope of this thesis, however, Figure 1-2 depicts the 
difference between all four types in terms of propulsion efficiency and flight Mach number, $M_{o}$. The interested reader can consult (Huenecke, 1997).

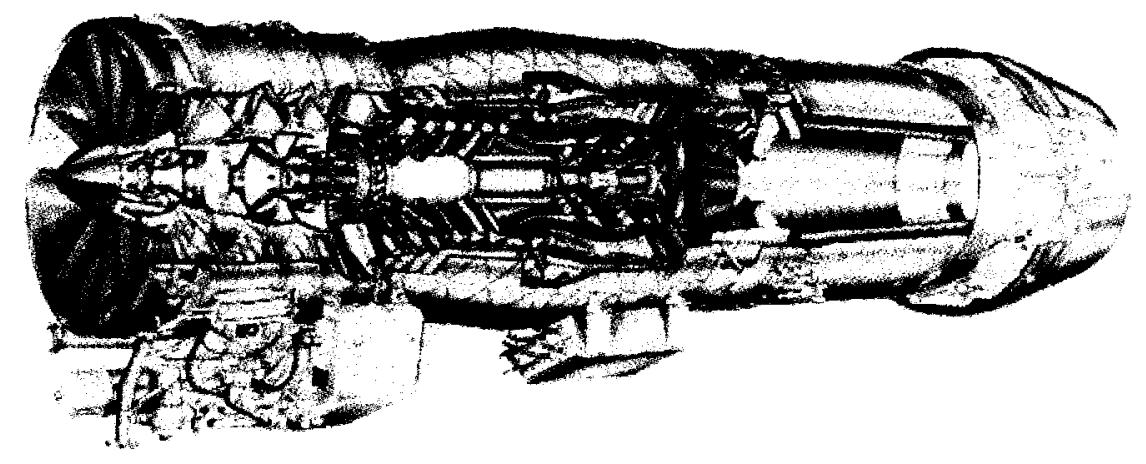

Figure 1-1: The M88-2 gas turbine engine (snecma, 2005)

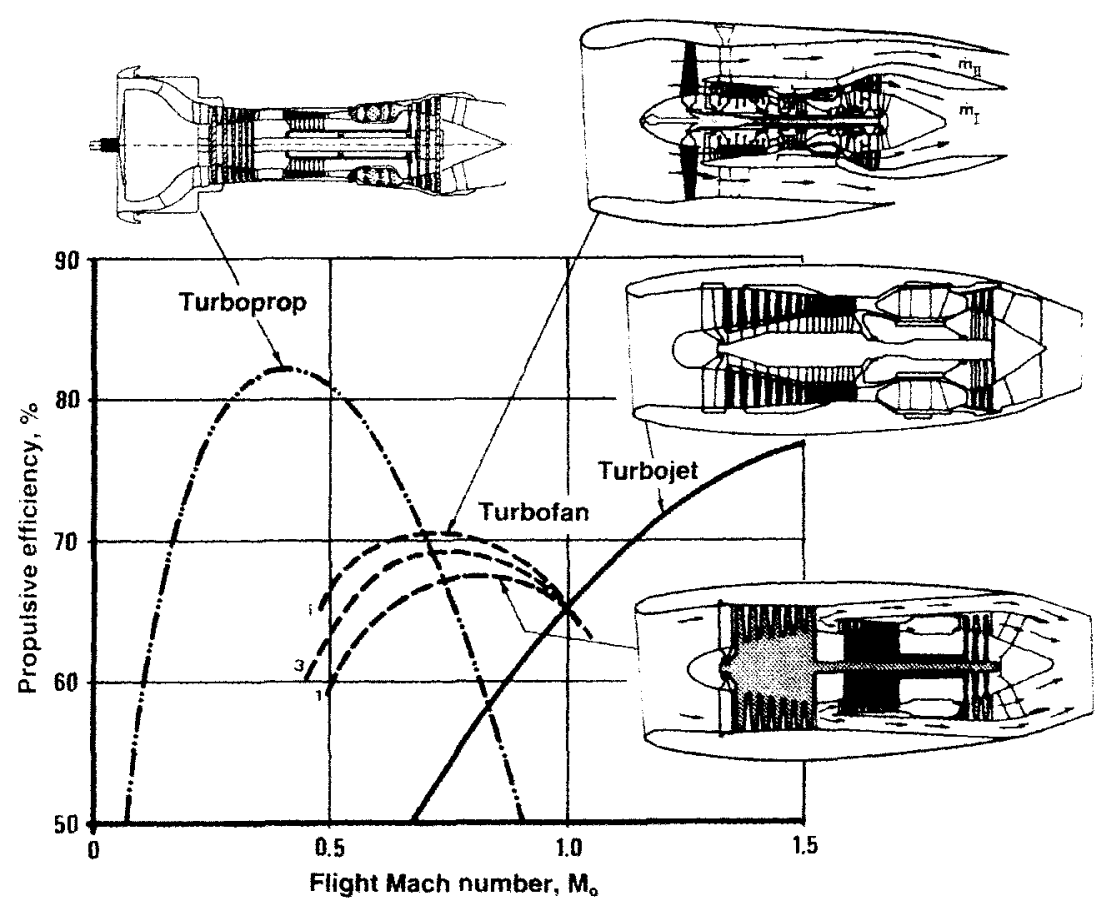

Figure 1-2: Propulsion efficiency characteristics of aero-engines (Huenecke, 1997) 
As mentioned earlier in this section, the stringent demand for response time and speed of the GTE requires a rigorous control strategy. Therefore, the subsequent sections will focus on the technologies used to control gas turbines engine.

\subsection{Gas turbine engine control}

Control of a gas turbine engine in its basic form can be represented as fuel flow metering to the combustor nozzles as a response to lever movement or power demand. This simple scheme, based on computing the exact amount of fuel needed for an engine to produce the necessary power, is rarely used without introducing limiting factors, such as limiting temperatures, maximum spool speeds, and fuel schedules. Since the first GTEs were built, control technologies have steadily improved from a simple hydromechanical fuel metering valve and a speed governor to a more robust and complex FADEC. The scope of the control system has evolved, in parallel, from a restricted propulsion (thrust) control to more diverse and automated onboard-engine diagnostic functions (or health management technologies) relying on defined mathematical models to monitor engine health and provide advanced prognostics and post-flight troubleshooting and maintenance support (Garg, 1993). Modern aircraft control philosophy is mostly directed towards more versatile controllers performing the complex task of real-time engine supervision and control coupled with engine health management software listed under "intelligent aerospace propulsion systems"(NASA terminology). By definition, the key functions of these intelligent systems are to increase the engine components' efficiencies through active control, advanced diagnostics and prognostics to enhance component life and reduce the frequency of maintenance intervention, and 
distributed control with arrays of smart sensors and actuators in an adaptive faulttolerance architecture. The advanced gas turbine designs have been developed under two conflicting constraints: the thrust-to-weight ratio criterion (necessitating high specific fuel consumption at high accelerations), and fuel economy and environmental considerations (i.e., enhanced performance and affordability, safety, and reduced environmental impact). This has resulted in the development of more stringent control laws integrating complex functions to cope with the nonlinear nature of the machinery while meeting the conflicting demands. In the absence of active and adequately responsive control algorithms, the structural integrity of the gas turbine components can be easily compromised; surge or stall for instance can occur in the compressor in less than 50 milliseconds, the overheating of the turbine can be effected in less than 0.25 seconds, while the turbine can over-speed in less than 2 seconds (Giampaolo, 1997).

Before going any further in our discussion of the control strategies or methodologies, we will introduce in the following section the propulsion control requirements for modern gas turbine engines.

\subsubsection{Propulsion control background}

A simple engine control system consists of a metering valve which regulates the fuel flow to the engine in response to shaft power or thrust demand while adapting to external disturbances (air flow perturbation, changing inlet pressure or temperature conditions). The block diagram in Figure 1-3 depicts this simple fuel control system. It is worth noting that during flight the thrust of the engine is difficult to measure. Since 
thrust can be proportioned to shaft speed and engine pressure ratio, a commonly accepted control variable is shaft speed $(\mathrm{N})$ or engine pressure ratio (EPR). Gas turbine performance in terms of thermal efficiency is subject to the varying operating conditions of inlet temperature and pressures (ambient conditions, altitude and speed variations), these changing conditions pose challenges from a control perspective. To illustrate these challenges, the fuel flow (Wf) and fuel ratio unit $\left(\mathrm{Wf} / \mathrm{P}_{3}\right)\left(\mathrm{P}_{3}\right.$ is the compressor exit static pressure) over the engine shaft speed $(\mathrm{N})$ is introduced in Figure 1-4.

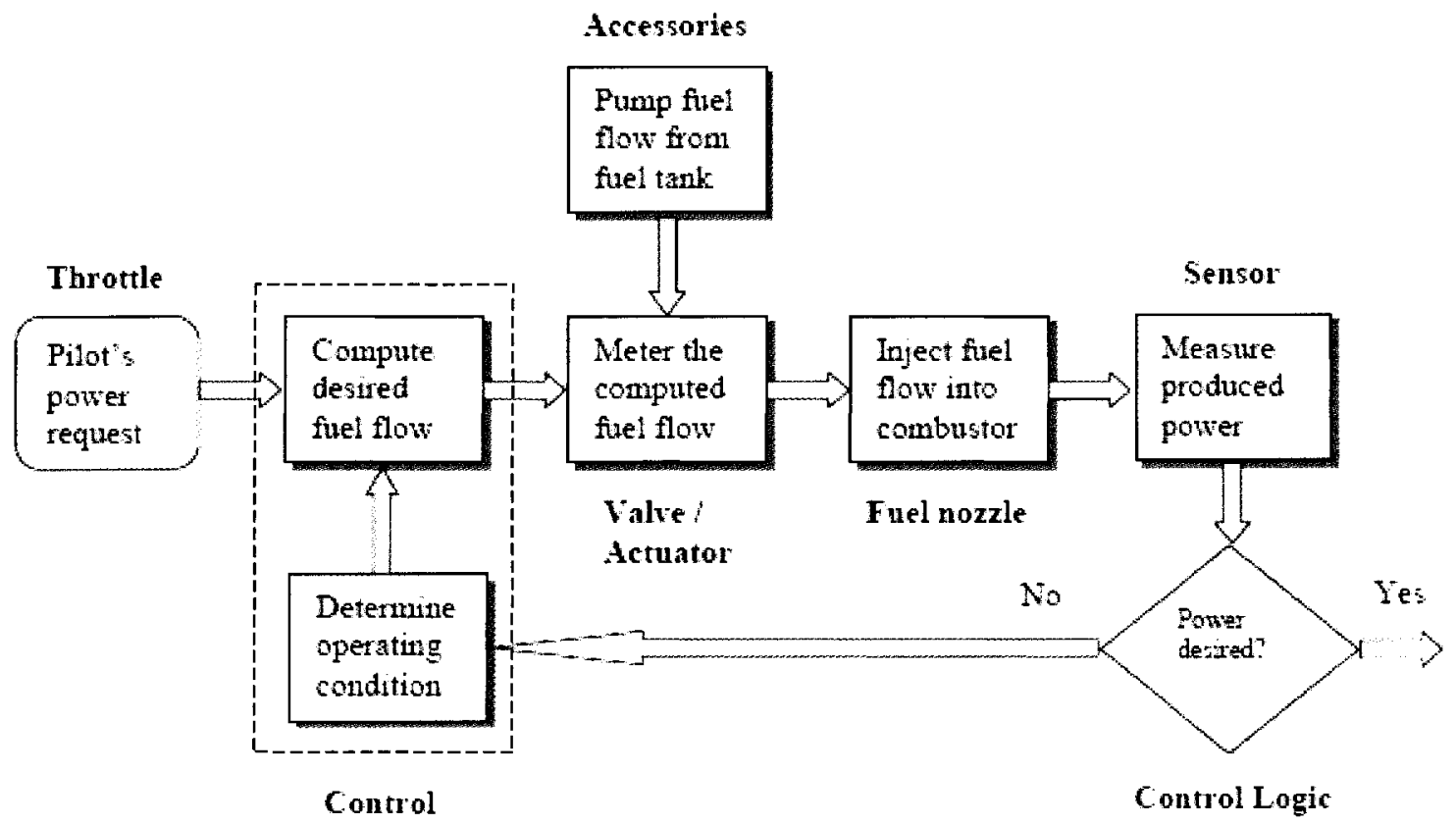

Figure 1-3: Diagram of simple engine control system (adapted from Sanjay, 2003) 


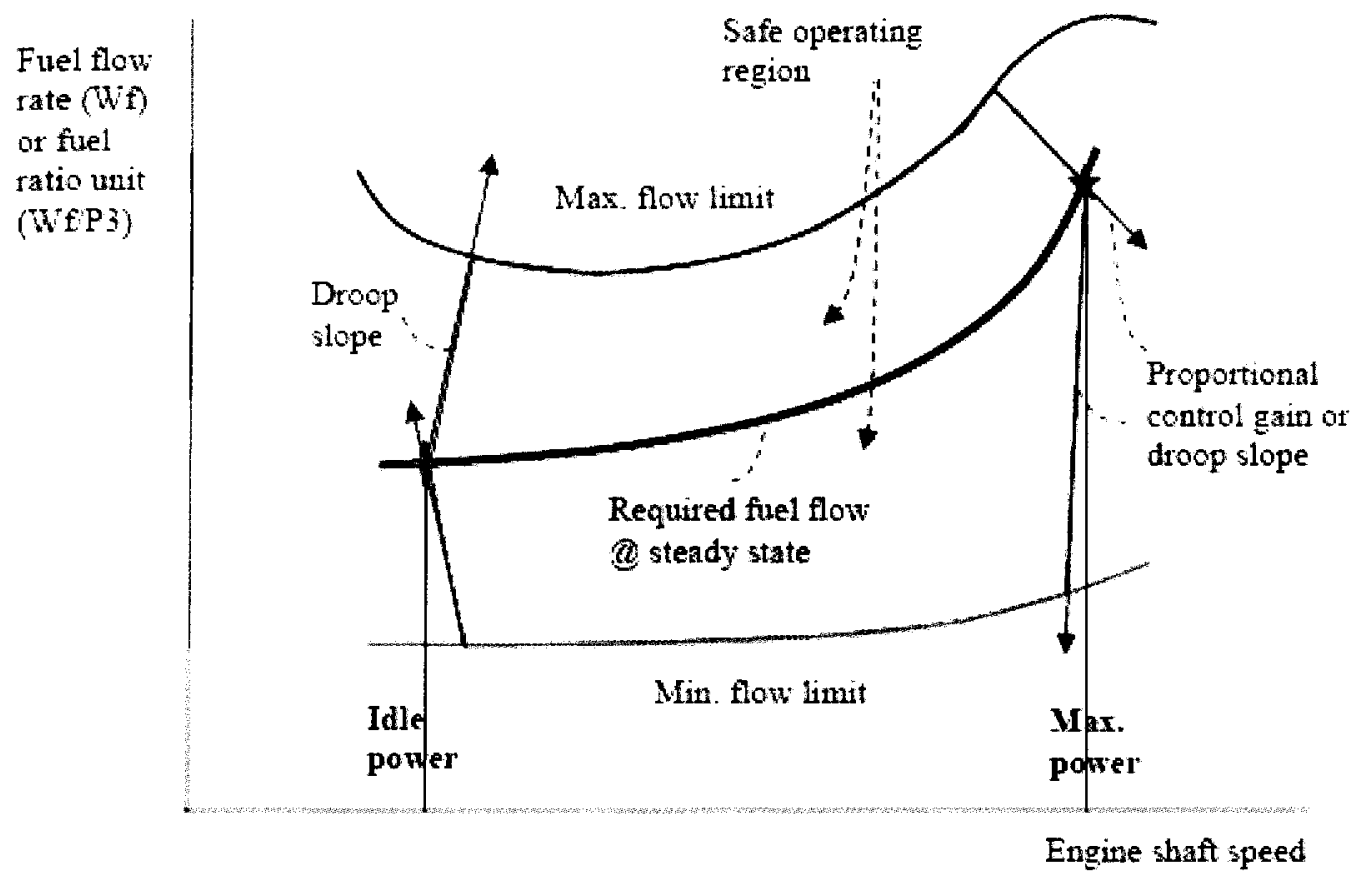

Figure 1-4: Gas turbine fuel control system operating boundaries (adapted from Sanjay, 2003)

As can be seen from Figure 1-4, a safe operating region for the gas turbine is highlighted in the shaded area of the graph. After engine startup, the transition from one operating point to the other is accomplished safely within this region bounded by the maximum-minimum fuel flow limits or fuel flow acceleration schedule (a path limited by compressor surge, over-temperature and over-speed of turbine) or deceleration schedule (fuel flow limited by flameout). The reason for adopting an acceleration schedule is to achieve the loading of the turbine from idle (no load) to maximum (full load) within a predefined time frame (Giampaolo, 1997). The four straight lines shown in Figure 1-4 intersecting the idle and maximum power points are the droop lines identifying the proportional control gains $(\Delta \mathrm{Wf} / \Delta \mathrm{N})$ at these power points. In actual controllers, the 
integral control component is used to enhance system robustness and the derivative component is applied to improve the transient performance. Both control laws induce non-rectilinear trajectories between a power setpoint and a fuel control limit (maximum limit or minimum limit).

\subsubsection{History of GTE control technologies}

Early GTEs relied on a simple hydro-mechanical control mechanism consisting of lever, cam, servos, and speed governor. As more complexity was introduced with highperformance engines and high speed setpoint computers were made available, electronic and digital controllers were developed to gradually substitute the early hydro-mechanical controllers. The first electrical control device was successfully implemented on the Theseus engine in 1946, and consisted of a throttle positioner that replaced the conventional mechanically linked throttles (Sanjay, 2003). The main reason for this change was the lack of precision of the mechanical linkages due to bending. The early gas turbines with open-loop throttles lacked the over-temperature protection option which subjected the engine to heat damage and to eventual fires. As a result, thermocouples were fitted in the jet pipe to monitor the exhaust gas temperature. Other limitations, such as maximum spool speeds and stresses affecting the structural integrity of the blades and shafts were monitored under the over-speed control algorithms. In 1951 General Electric (GE) proposed its early "SPEEDTRONIC," which was coupled to the LM2500 Marine gas turbine engine. The adjustable power-turbine nozzles of the engine provided an integration of nozzle angle with fuel setting to achieve optimal speed and temperature 
operating conditions (Woodward, 1975). However, it was not until 1956 that the first full-authority analog engine controller was developed to fit the Pretous engine in the Britannia aircraft. Another system, a comparable controller, was adopted by the Gnome helicopter engine shortly after (Andrew et al., 1999).

The methods of development adopted by these technologies are discussed in the next sections.

\subsubsection{Control methodology}

\subsubsection{Time-domain based methodology}

The GEI-A was the first US engine built in 1942 to use a hydro-mechanical governor metering the fuel flow to the combustor chamber proportional to the variation of the turbine setpoint speed and actual speed. The control law originally introduced was based on the frequency response technique followed by the time-domain step response analysis method. The steady-state performance was obtained from the cycle calculation using component performance maps derived from test results, constant specific heat gas properties, and an iterative process for balancing internal engine flow and heat transfers. The dynamic behaviour of single-shaft engines at NACA Lewis Laboratory in 1948 revealed that the transfer function of the fuel to the engine speed can be represented by a first-order lag linear system with time constant (Otto and Taylor, 1950). In 1951 Pratt \& Whitney (PW) introduced the first two-spool afterburning turbojet engine, the J-57, and achieved supersonic speed with a YF-100. The control system design was pivoting around the frequency response method with gain and phase margin analyzed as the 
leading design parameters for closed-loop system stability. The step response method was employed to ensure stability and performance in the entire operating envelope. Successive loop closure was the technique used invariably to decouple the effects among multiple control variables.

A leap towards the linear state-space methods in control marked the early $1960 \mathrm{~s}$. Relying on the Newton-Raphson method developed by the Air Force Aero Propulsion laboratory to reduce the time needed to compute a design point using digital or analog computing techniques. The analog and digital electronic control units (ECU) were introduced in the early 1970 s to provide high-level supervisory or trim functions. These units calculated speed or temperature setpoints through the engine operating range. Early ECU incorporated 1) the digital Electronic Engine Control (EEC) for the PW F100, and 2) the analog Augmenter Fan Temperature (AFT) for the GE F101 (Sanjay, 2003).

A full-authority ECU is designed to control the entire operation of the engine from startup to shutdown according to input throttle command. The TFE 731 engine ECU and the F100 engine DEEC were the first analog and digital electronic controls simultaneously produced in the US. The TFE 731 ECU was a single-channel analog control with fixed fuel ratio maximum and minimum schedules and a hydro-dynamic control (HMC) as a back-up. The switching from analog to digital control was motivated by the need to improve on the mean time between failure for a given mean time between unscheduled removal and field maintenance. Subsequently, the flexibility of the digital software in complex computation and its capability in fault isolation through self-testing, coupled with a better ability for accurate control tuning did set the trend for the digital 
electronic controls. The major advantages of these electronic control units are summarized in point form as follows:

○ "Significant size and weight reduction for the same control functions

- Fast software update (laptop)

o Provide an unrestricted thrust lever movement

- Eliminate engine trim after any control component change

- Compatible with onboard monitoring controls

- Engine data recording

- Enhanced maintainability

- Diagnostic ability (fault isolation and flags)" (Sanjay, 2003).

The frequency domain methods of classical control theory based on the Inverse Nyquist Array (INA) method (McMorran, 1970) and the time-domain methods led by the Linear Quadratic Regulator (LQR) method were extensively being applied in the control of the GTE. Though, in recent years, time-domain methods based on the variations in linear optimal control methodologies - namely, Linear Quadratic Gaussian (LQG) (Athans et al., 1986) and H-infinity (Garg, 1993, Frederick et al., 2000) - were applied for controlling the aero-engine (Harefores, 1997). A breakthrough in aero-engine control technology was sought with the introduction of the multivariable control synthesis (MVCS) program (DeHoff et al., 1977) with assumed full-state feedback and perfect control performance. The Multivariable system relied on the LQR method and suffered from the limitations in solving complex nonlinear systems problems with linear optimal control methods. 
An obvious limitation of linear optimal control, mostly investigated under steadystate conditions, is that these systems fail to satisfy the design requirements when the state variables are at their actual limits. This downside can be overcome with nonlinear control laws allowing the output parameters to follow any course over the entire operational envelope and hence improving the GTE control efficiency. A method is described in (Lyantsev et al., 2004) which introduces multivariable local-optimal control systems using numerical algorithm with real-time optimization of control laws, and accounts for different characteristics of the GTE. In this method, the full derivative of the Lyapunov function is used as a local optimality criterion to ensure the asymptotic stability in the control system. The results showed that optimal multivariable control can be achieved with a high degree of decoupling between control loops while considering constraints such as actuator movement and speed limits.

\subsubsection{Expert knowledge system methodology}

In another method, based on Zadeh (1965), fuzzy logic was introduced by Mamdani (1975) who demonstrated the control action of a steam boiler plant using a linguistic fuzzy approach. Procyk and Mamdani (1979) developed a heuristic controller based on adaptive fuzzy sets to respond to varying process environments with the aim of optimizing the performance of the controlled process. Chi-Hua et.al. (1989) detailed a fuzzy logic control for a turbojet fuel control system application. Their work involved the implementation of a fuzzy controller on a microcomputer to simulate the fuel control system. They showed that the demand for fuel (simulated by a step signal), in response to engine speed, can be achieved by the designed fuzzy controller within one second. 
Balakrishnan et al., (1994) proposed a fuzzy logic controller based on a turbine entry temperature (TET) datummed approach for a single-spool jet engine. In their study, they aimed at correcting the fuel flow to the engine by a control action based on the rate of change of the error of the measured TET as compared to a preset value. The engine was under development and the implemented fuzzy control algorithm simulation results were comparable in terms of engine response for speed and thrust with reference to experimental data from Orpheus 703 engine tests.

The combination of fuzzy logic and artificial neural networks led Matsumoto (1997) to the development of a fuzzy controller for the startup cycle of a $235 \mathrm{MW}$ combined cycle power plant. Their target was to reduce startup time, NOx emissions and fuel consumption. Their strategy consisted of using the fuzzy rules to qualitatively assess the causal relation between operational constraints and the ideal schedule modifications at different ambient and operational conditions. As a result, a net saving of US\$12M/year in fuel cost was achieved.

The advantages of using fuzzy adaptive knowledge in control applications were pronounced by Zengqi (1996). Later, Delara (2002) developed a feedforward neurofuzzy speed controller for a turbogas unit and demonstrated the ability of the fuzzy controller to exceed the PI (proportional integral) controller performance in terms of reduced thermal stresses and confirmed the universal approximation property of the fuzzy system. It is clear from the literature that the advantages of FLC in terms of reduced development time and simplicity in implementation have given researchers strong motive to develop guidelines for the development methodology of fuzzy logic systems control. 
Consequently, a leading effort was made in Europe to define an FLC standard based on ISO-9000 general systems (Schram et al., 1997).

\subsection{Thesis presentation}

The present work is divided into 6 chapters, including the introduction. The focus of this thesis is to apply the fuzzy logic to control a gas turbine engine using a simulation model of the gas turbine engine derived from GasTurb software. It also introduces the subject of gas turbine modelling using artificial neural network techniques. Chapter 2 covers a literature review on gas turbines and the developments in their control modes. It includes a description of the gas turbine engine control strategies and methods that are in use today.

Chapter 3 examines fuzzy logic systems in order to elucidate the implementation of expert knowledge systems in control problems. Two inference systems, the Mamdani and the Sugeno fuzzy inference engines, are examined and their application in the proposed fuel schedules is discussed.

Chapter 4 introduces the artificial neural network (ANN) and briefly covers its application in solving engineering problems, one of which is the modelling of gas turbine engines.

Chapter 5 is a simulation analysis. It includes the simulation models employed in this study: GasTurb and Zero-Emission GT. It discusses the design of fuzzy fuel schedules (acceleration and deceleration) for a $1 \mathrm{MW}$ gas turbine engine model simulated in GasTurb. It also represents an approach using a Sugeno fuzzy inference engine as the 
FLC to control a gas turbine engine modelled with Zero-Emission GT in a predefined range of operation. The fuzzy controller performance is then assessed under a defined operating conditions and the performance of a PID controller is shown. It tackles the dynamic system modelling techniques and the application of a feedforward artificial neural network in modelling a gas turbine engine. It shows the results of duplicating the spool speed, TIT and fuel-flow relationships of a $1 \mathrm{MW}$ gas turbine engine using data generated in GasTurb by an artificial neural network.

Chapter 6 presents the conclusions and the recommendations for future work. 


\section{Chapter 2}

\section{Literature review}

Gas turbine technology has steadily evolved over the years to a top standard in reliability, efficiency, and power-to-weight ratio. Such development promoted this power plant in many engineering fields of applications, mainly in the aircraft and power generation industries. The engines high power output or thrust-to-weight ratio and efficiency made them the number one choice for marine propulsion. The potential of GTEs to burn different grades of fuels positioned them as the preferred means for electric generation facilities relying on combined heat cycles, dry low NOx and biomass gasification technologies.

A thorough introduction to gas turbine theory is included in (Saravanamuttoo et al., 2001). Before an actual engine is built, a model of the GTE must be developed. Engine models are required both in the development and in operational stages of the gas turbine. Model complexity differs according to the application in question. One approach is modelling based on mass, momentum and energy balance. This method describes the spatial flow of gas dynamics in each section of the gas turbine by assuming the thermodynamic state at the corresponding section to be spatially constant but temporally varying (by using the fundamental conservation equations). Therefore, it allows the performance of the gas turbine to be analyzed by a set of ordinary differential equations that can be easily coded in a computer simulation program. The sections of a 
typical gas turbine could include the compressor, combustor, and turbine. Nonetheless, thermodynamic models are complex and nonlinear. It is therefore common to linearize the models around a series of operating points and then carry out a model-order reduction, in order to arrive at models which are suitable for control system design. Validation of the performance with real engine data will have to follow. However, identifying the nonlineraity in gas turbines for control purposes poses a serious problem. It necessitates detecting the response at different operating points covering the range of speeds from idle to full load, which in itself is a complex task since the sources of nonlinearities are different. For instance, there are nonlinearities associated with the variation of the engine dynamics with operating conditions (Saravanamutto, 1992) and the nonlinear dynamic changes at different engine conditions: e.g., blade tip clearances effect on the dynamics during acceleration or deceleration (Pilidis, 1986). A different concept of modelling is the quasi-static approach that relies on the collection of real-time steady-state gas turbine performance data (Hung, 1991). In this modelling technique, the transient thermodynamic and flow dynamics are assumed to progress ad infinitum along the steady-state performance curves. However, with this method the dynamics of the gas turbine over the entire operating envelope (from startup to shutdown) may not be captured.

The method just described falls under the category of system identification, which refers to an algorithm for constructing or defining a model from data and is thought to provide the needed information about engine behaviour for control purposes. In practice, identifying aircraft gas turbine dynamics is mostly done with "wobble" tests that use 
single sines at different frequencies as excitatory waves to the engine. Bode plots are then produced to account for all the gains and phase shifts at each frequency (Ljung, 1996). However, this procedure is costly and time-consuming. Evans (2001) discusses three approaches for system identification, highlighting their advantages in terms of lower costs and shorter test run times. These are "the multisine test signals and frequency-domain identification techniques" for enhanced linear modelling, "the extended least squares with optimal-smoothing" for detecting time-varying linear models, and the "multiobjective genetic programming" for identifying nonlinear model structures.

Another emerging approach is modelling according to an artificial neural network (ANN). This expert system, which has excellent function approximation capabilities (Döhler, 2000), is widely used in applications requiring the mapping of input vectors to output vectors (Rao et al., 1995). Modelling in the time-domain requires defining the relationships between past input output data and future outputs. Since GTEs are nonlinear systems, it would be feasible to identify their performance at different operating points by a set of input output engine data relations. While the inherent dynamic nonlinearity can be captured with the sigmoid or hyperbolic tangent functions embedded in the activation unit of $\mathrm{ANN}$, the structure of the ANN and the training algorithm provide accuracy over the entire operating envelope of the GTE (Chiras et al., 2002). In this thesis the transient behaviour of a GTE is modelled using an ANN.

After a satisfactory dynamic model of the engine is generated, control engineers proceed in deriving a suitable control strategy. The next section will provide insight into the control functions and methodologies available for GTE. 


\subsection{Background}

Controlling the gas turbine requires prior knowledge of its configuration and hence of the constraints imposed by the design and envelope of operation. For industrial or power generation applications, for example, the response time depends on the inertia of the driven equipment. In aircraft engines, the change in ambient conditions (temperature and pressure) can adversely affect the operating performance of the gas turbine. Under any conditions, the control must be able to limit the speed of acceleration during rapid power changes to avoid excessive heat generation in the combustor (which can cause the turbine blade to overheat) or a surge in the compressor (which can severely damage compressor blades and stator vanes). In addition, it must eliminate the flameout phenomenon caused by sudden deceleration, which can be detrimental to the turbine (if repeated frequently it can cause thermal stress concentration in the turbine blades). In modern gas turbines, such as variable geometry engines, more controllable elements with advanced nonlinear control techniques are sought to guarantee the full potential benefits of the engines. Nonlinear systems control, of gas turbines in particular, necessitates the definition of gain schedules covering their operating range. Accordingly, a set of performance criteria is defined at each design point and the controller has to ensure a smooth transition between these points. These criteria include transient and steady state accuracy, disturbance rejection, stability, stall margin, and structural integrity. While these demands can be met from an operational point of view, the task of compromising between optimizing on the power or thrust and specific fuel consumption, speed of 
response and limiting the rate of thermal stresses are constraints imposed on the overall control strategy.

The subsequent section summarizes the control functions for a typical GTE.

\subsubsection{Gas turbine engine control function}

The gas turbine control system performs control functions, protection functions, alarms, and communication functions. In general terms, it must be able to execute the following primary tasks.

- Ensure proper startup and acceleration to idle speed;

- Ensure safe transient performance and engine acceleration according to power demand;

- Govern the engine at any given steady-state operating speed;

- Manage the fuel demand to the engine with fuel acceleration and deceleration schedules to limit compressor surge and turbine overtemperature or combustor flameout while minimizing fuel consumption or peak thermal stresses;

- Limit engine speeds and pressures to permissible values; and,

- The shutdown of the engine.

Secondary functions of the controller include engine bleed, power extraction fan, compressor tip-clearance control, and compressor stator blade settings for variablegeometry designs. For a typical GTE control mode, the acceleration mode is the most critical function of the control system. Because of stringent flight safety requirements, aircraft gas turbines must be able to accelerate from 15 to 95 percent thrust in only 5 seconds (MacIsaac, 1986). At these acceleration rates the safe limits of operating 
temperature and compressor surge can be easily exceeded.

An overview of the most critical controlled stage of the GTE will be provided in the following subsection.

\subsubsection{Transient performance}

As mentioned earlier, compressor surge or stall and turbine overheating must be avoided at all costs. A surge is a condition of repeated flow interruption at the compressor end causing a reverse upstream propagation of the air flow for short time intervals. A reduction in the mass flow rate or too much rotation of the compressor blades, or both, contributes to the occurrence of compressor surge. The aerodynamic phenomenon caused by the aerodynamic stall of one or more blade, or aerodynamic instability, can induce high compressor vibration and combustor flameout; both conditions are detrimental to the gas turbine especially for aero-engine applications.

Material characteristics and blade tip cooling design sets the acceptable limits for the turbine blade operating temperatures at about $1350^{\circ} \mathrm{C}$ in modern GTEs (Boyce, 2002). The temperature in the combustion process is regulated by the injection of fuel per unit time; high rates of fuel will cause a rapid increase in the temperature at the outlet of the combustor and consequently at the inlet turbine blades. Such a phenomenon may lead to an overheating of the blades causing advanced blade deterioration and possible failure.

The ultimate control law for fuel flow relies on a closed-loop control for compressor anti-surge (minimum acceptable flow) and turbine inlet temperature. Although, extensive research is being conducted to detect and control surge (Blotenberg, 
1995) the start of a surge often cannot be detected quickly enough, nor can it be compensated accurately. It is worth noting that anti-surge controllers depend on electronic hardware with transmitters and instruments that incorporate noisy error drifts, and must compensate for the changes in the surge line due to compressor fouling. This by itself is a very complex and costly task. For instance, a closed-loop control to avoid surge requires a way for sensing the onset of flow separation. This can be achieved by either estimating the separation by inserting static pressure taps along the suction surface of the vanes (extremely costly) or by introducing a pressure transducer in the casing at 85 percent cord next to the vane suction surface (Sanjay, 2004). As for the turbine temperature, a common practice is to measure the gas stagnation temperatures at the outlet of the turbine via a set of thermocouples. The turbine inlet temperature (TIT) is then calculated using temperature thermodynamic relationships. Current research is moving towards incorporating pyrometric readings at the TIT side for the most advanced turbo-machineries.

In practice, fuel schedules are introduced as limiting factors in the control strategy to hedge against compressor surges and turbine overheating. These schedules comprise acceleration and deceleration limits, as will be discussed in the coming section.

\subsubsection{Acceleration and deceleration schedules}

An open-loop control of gas turbine acceleration is commonly employed to overcome the compressor surge problem. The acceleration schedule can be defined as the maximum permissible transient response of the gas turbine within an interval of 
operation bordered by the compressor surge line (the limit of compressor flow stability). The deceleration schedule, or the guaranteed minimum fuel limit, normally prevents the combustor from flameout during deceleration.

A typical compressor performance map is shown in Figure 2-1. This map shows the surge line, surge margin, and the steady-state operating line with constant aerodynamic speed lines plotted on a mass flowrate and pressure ratio diagram. The stall line or surge line can either be determined theoretically from the thermodynamic model of the gas turbine or by real surge measurements taken at different operating speeds or guide vane positions for a commissioned engine. Once the surge margin (distance between the operating line and the surge line) is set, the acceptable acceleration rates can be established and the acceleration schedule formulated. 


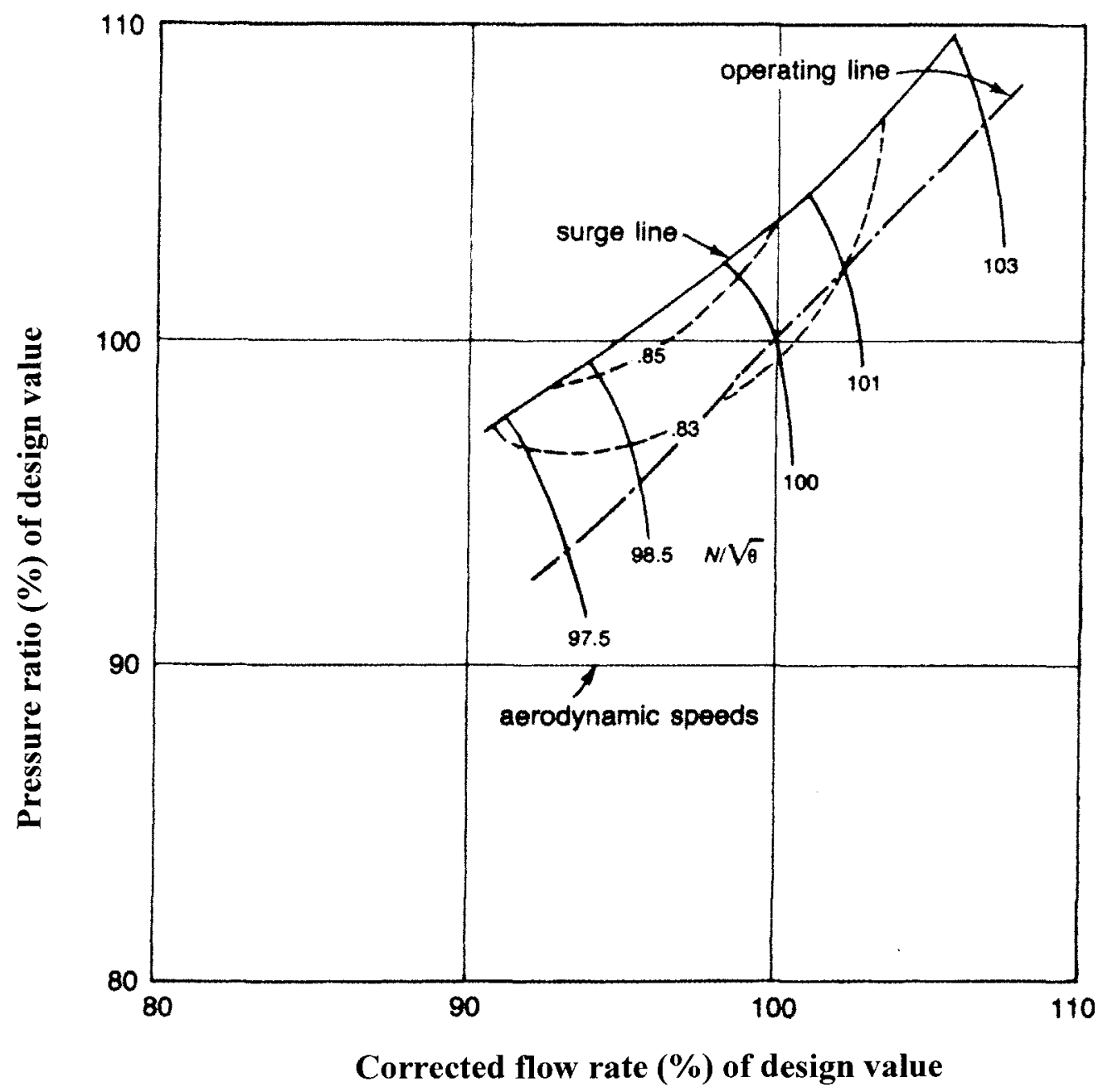

Figure 2-1: Typical compressor map (adapted from Boyce, 2002)

The acceleration schedule is mapped as a curve on a fuel flow (Wf) or fuel flow divided by discharge compressor static pressure $\mathrm{Wf} / \mathrm{P}_{3}$ (called the fuel ratio unit), versus compressor rotating speed graph (shown in Figure 1-4). In practice, and to avoid ambient leverage on gas turbine performance, the mathematical representation of acceleration schedules is realized in non-dimensional equations. A general non-dimensional form for GTEs is: 


$$
\frac{W_{f}}{\delta_{1} \sqrt{\theta_{1}}}=f\left(\frac{N}{\sqrt{\theta_{1}}}\right)
$$

where

and

$$
\theta_{1}=\frac{T_{1}}{T_{\text {ref }}}
$$

$$
\delta_{1}=\frac{P_{1}}{P_{\text {ref }}}
$$

These non-dimensional equations provide a link between the actual operating conditions and the reference conditions of GTEs. As such, the map shown in Figure 2-1 can be used to depict the performance of the GTEs at different operating points.

The concluding step in the acceleration/deceleration schedule is to test and validate the analytical model on a test rig engine. Correction and fine-tuning is effected to ensure that the performance requirements, in terms of avoiding surge and overheating while maintaining the stringent demand for thrust or acceleration, are met. These schedules will be the focus of the control strategy adopted in this thesis.

Now that the gas turbine control methods have been introduced, the next two chapters will detail the expert knowledge methodology, namely the fuzzy logic method and artificial neural network and their application in the control and modelling of gas turbine engines. 


\section{Chapter 3}

\section{Fuzzy logic}

A fuzzy logic system can be defined as a clear set of rules mapping human logic to a numerically structured process defined by sets and membership functions (Sun et al., 1997). This expert knowledge system is constructed on the basis of linguistic facts and activated by proposals generating conclusions. It is primarily used for tackling problems of uncertainty and nonlinearity in the areas of modelling, robotics, image processing and machine vision (Hall et al., 1992), pattern recognition (Carpenter et al., 1991), medical diagnosis, constraint propagation and optimization, finance, fuzzy neurocomputing and process control. For the control part, Zadeh (1988) states: "But what is striking is that its (fuzzy logic) most important and visible application today is in the realm not anticipated when fuzzy logic was conceived, namely, the realm of fuzzy-logic-based process control."

\subsection{Fuzzy sets}

Fuzzy sets are a generalization of the fuzzy conventional set theory first proposed and outlined by Zadeh in 1965 . These fuzzy sets are mere deterministic sets with imprecise boundaries. By that, it is meant that fuzzy logic is a system that formalizes approximate reasoning by formulating mathematical relations coupled with linguistic facts to represent a real model (Jamshidi, 1997). 
To elaborate, fuzzy logic is constructed on the basis of linguistic facts and activated by mathematical proposals generating logical conclusions. Fuzzy sets introduce new concepts to represent the degree of membership of a given variable in a defined domain. In mathematical terms, fuzzy sets and crisp sets are distinct. On one hand, the crisp set (i.e. containing objects that satisfy precise properties required for membership) allows a membership $\mu_{A}(\chi)$ of a variable $\chi$ for a crisp set $A$, as subset of the universe $X$, to be defined by:

$$
\mu_{A}(\chi)=\left\{\begin{array}{l}
1 ; \text { if } \chi \in A \\
0 ; \text { otherwise }
\end{array}\right.
$$

where an element $\chi$ is either a member of the crisp set $A$ or is not.

On the other hand, fuzzy sets are characterized by a membership function which associates to each point in the domain of definition a real number between 0 and 1 . In other words, fuzzy sets allow more tolerance for imprecision than crisp sets do, in terms of the representation in a given set. Figure 3-1 depicts the crisp and fuzzy membership functions of a subset $A$.

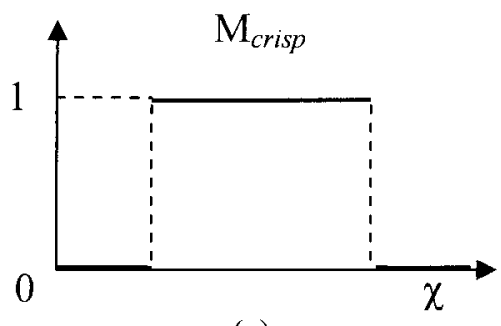

(a)

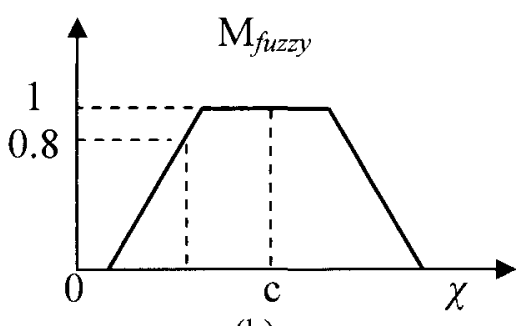

(b)

Figure 3-1: Membership function for crisp and fuzzy subsets of $A$, in (a) crisp set and (b) fuzzy set 
Figure 3-1(a) shows that every real number $\chi$ is either in $A$ or is not since the membership function $\mathrm{M}_{\text {crisp }}$ maps all numbers $\chi \in A$ onto the two points $(0,1)$. By contrast, part (b) of the figure shows that numbers closer to $\mathrm{c}$, the logical truth, can be represented by a fuzzy membership function $\mathrm{M}_{f u z z y}$ and this is more representative of real problems, where uncertain information or vague knowledge and imprecise data are present.

In real life applications, the "truth" may not be a crisp or exact value for the membership of a variable $\chi$. For example, this is the case in the temperature regulation of a given process where "colder or hotter than" a given preset temperature notion can describe the variation of a controlled variable. In this regard, a fuzzy set allows more flexibility in categorization than the crisp set.

In a formal way, a fuzzy set designates a degree membership between 0 and 1 to a crisp value $\chi$ using a membership function $\mu_{A}(\chi)$, defined on a fuzzy set $\tilde{A}$ by

$$
\mu_{\tilde{A}}(\chi): X \rightarrow[0,1]
$$

The fuzzy set $\tilde{A}$ is a crisp set of ordered pairs $\chi \in X$ and $\mu_{\tilde{A}}(\chi)$ :

$$
\tilde{A}=\left\{\left(\chi, \mu_{\tilde{A}}(\chi)\right) \mid \chi \in X\right\}
$$

Or as first introduced by Zadeh (1965):

$$
\tilde{A}=\sum \mu_{\tilde{A}}\left(\chi_{i}\right) / \chi_{i}
$$

For example, for a linguistic variable such as price, the fuzzy sets could be selected as expensive, moderate, and cheap (linguistic term), and each linguistic term has its membership function (trapezoidal, bellshape, triangular, etc.). In a numeric form, a crisp 
value of $\$ 100$ can result from a combination of three membership functions; $75 \%$ expensive, $25 \%$ moderate, and $0 \%$ cheap.
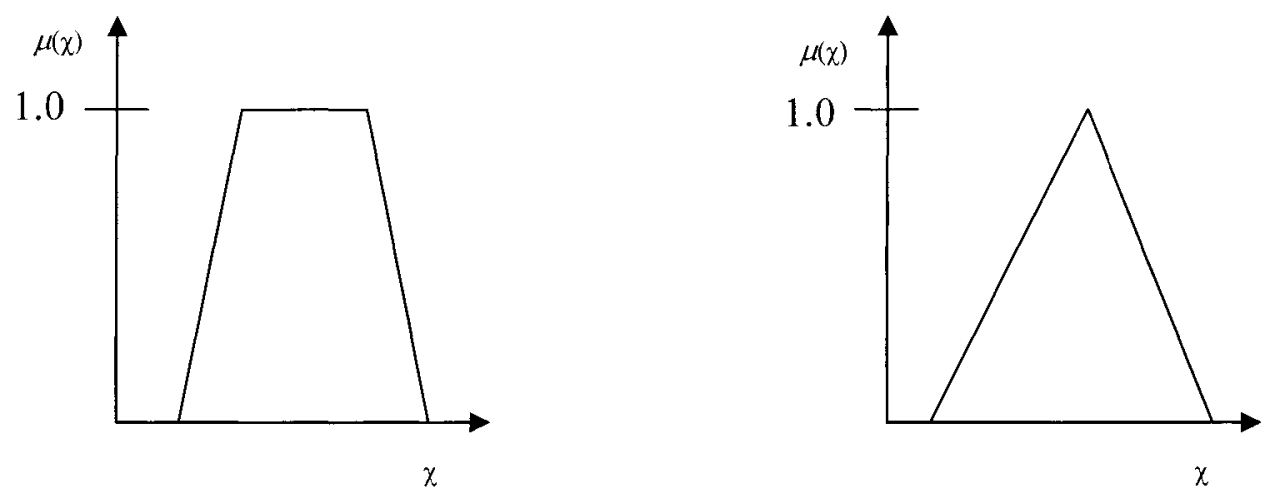

Figure 3-2: Typical membership functions used in fuzzy logic control

Membership functions used in fuzzy logic can assume many shapes. However, typical shapes used in control applications are the triangular and the trapezoidal shapes (Figure 3-2). Other membership functions could be of the form of the Gaussian distribution function, the generalized bell, pi-shaped, Z-shaped or sigmoid curve. The selection of the membership functions depends on the application and ease of computation. For real-time applications, the triangular or trapezoidal functions are the preferred choice because their evaluation time is shorter than the more mathematically elaborated functions.

Mathematical operators such as union, intersection and complement can be applied to fuzzy sets, as inference rules, but under different definitions than the classical mathematical sets. Typically, T-norm operators are employed for intersections, and T- 
conorm (S-norm) operators are used for unions. A T-norm operator is a two-consign function satisfying boundary, commutativity, monotonicity (the closer the value of $\chi$ to $c$, the closer $\mathrm{M}_{f u z z y}(\chi)$ to 1 , as in Figure. 3-1) and associativity conditions. This includes the following products: minimum, drastic, algebraic and bounded. A T-conorm (S-norm) operator is, on the other hand, maximum, algebraic-sum, bounded-sum, and drastic-sum. Both "norms" possesses monotonicity, commutativity and associativity properties:

$$
\begin{aligned}
& T(a, b)=T(\mathrm{~b}, a) ; \quad S(a, \mathrm{~b})=S(\mathrm{~b}, a) \\
& T(a, T(b, c))=T(T(a, b), c)
\end{aligned}
$$

Satisfying the given boundary conditions:

$$
\begin{aligned}
& T(a, 1)=T(1, a)=a \\
& S(a, 0)=S(0, a)=a
\end{aligned}
$$

The implications of these operators in fuzzy reasoning occur at the inference rules level. These rules include the categorical rules and can be of the form (from Zadeh, 1973 and 1988):

Intersection operation:

$$
\mu_{A \cap B}(\chi)=\min \left(\mu_{A}(\chi), \mu_{B}(\chi)\right)
$$

Union operation:

$$
\mu_{A \cup B}(\chi)=\max \left(\mu_{A}(\chi), \mu_{B}(\chi)\right)
$$

Complement $\bar{A}$ of the fuzzy set $A$ :

$$
\mu_{\bar{A}}(\chi)=1-\mu_{A}(\chi)
$$

These rules for inference in the fuzzy logic are used in this thesis.

Now that the fuzzy set has been described, the next section will deal with how to 
build a model using fuzzy logic reasoning for control purposes.

\subsubsection{Fuzzy reasoning}

A fuzzy logic process includes three stages; fuzzification, fuzzy inference engine, and defuzzification. Figure 3-3 depicts the flow of information processing by the FLC.

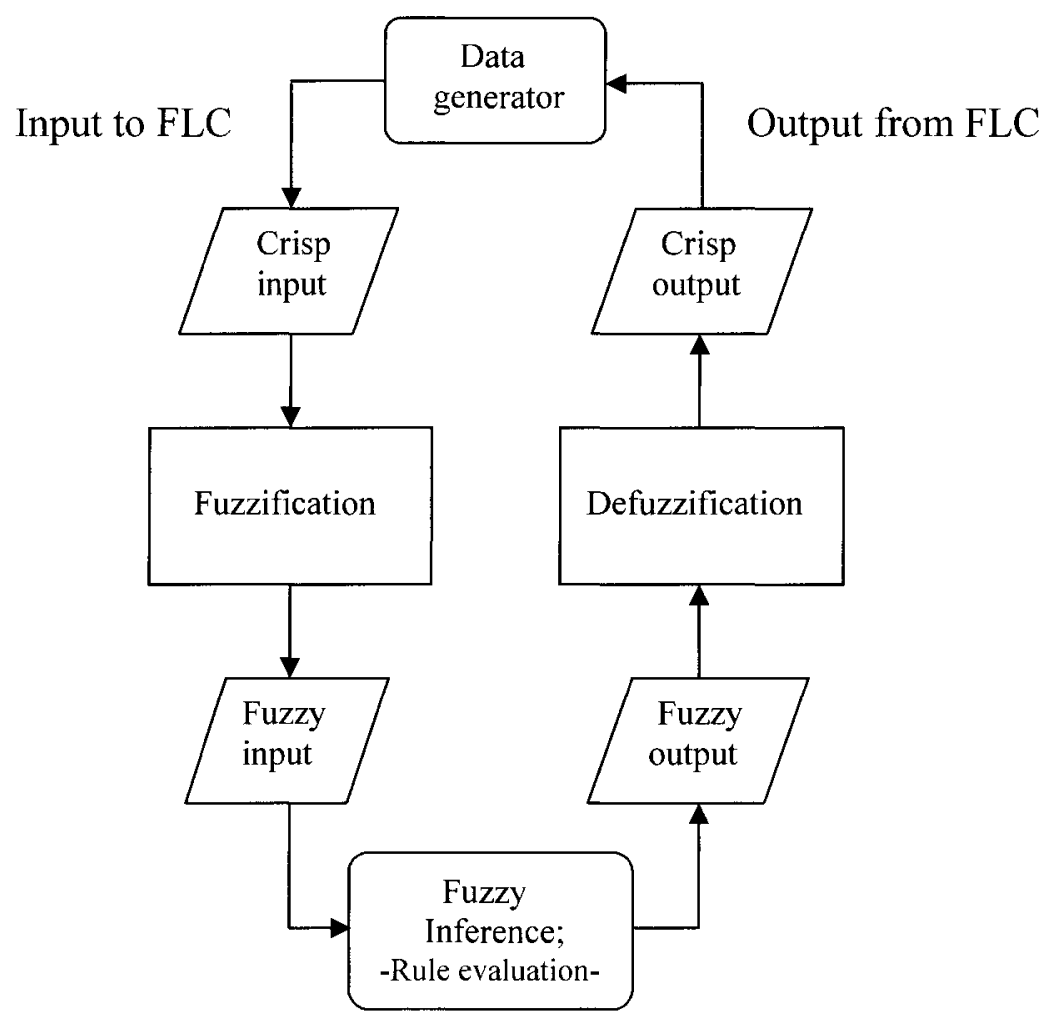

Figure 3-3: Flowchart of fuzzy logic controller

The fuzzification stage transforms the input vector of a given crisp value into a fuzzy input variable by evaluating the membership functions of each linguistic input variable; as shown in Figure 3-4. 


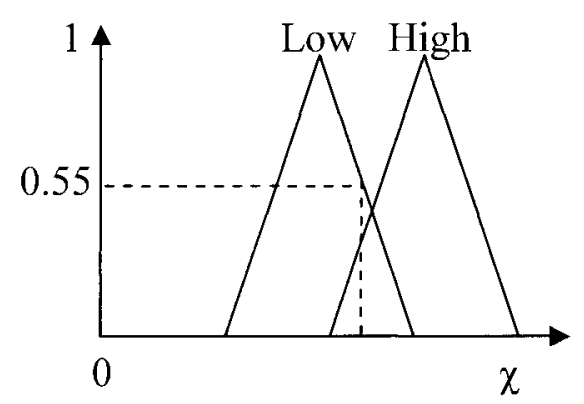

Figure 3-4: Fuzzy input variable

For example, for an input $\chi$, the membership function corresponding to the linguistic input variable Low is 0.55 .

This step is followed by the fuzzy inference engine or the Fuzzy Inference System (FIS) which decodes a set of if-then rules with linguistic variables as premises and conclusions or as antecedents and consequents:

$$
\text { If } \chi \text { is } A_{i} \text { then } z \text { is } B_{i}
$$

where $\chi$ and $z$ are the linguistic input and output variables in a rule-base of size $M$, and $A_{i}$ and $B_{i}(i=1,2, \ldots M)$ are fuzzy sets representing the linguistic terms of $\chi$ and $z$. The premise or antecedent is $\left\langle\right.$ If $\chi$ is $A_{i}>$ and the conclusion or consequent is $\left\langle\right.$ then $z$ is $B_{i}>$. For example, if price is moderate then buy more.

The following step in the fuzzy process would be the formation of a fuzzy inference engine (or process) implying a rule firing strength evaluation, an implication function calculation and an aggregation process. The degree to which each of these constituents affects the fuzzy result will be scrutinized in the subsequent section. 
To exemplify the fuzzy process, we can look at any given rule with its antecedent composed of $m$ propositions connected with fuzzy operators (union and intersection). We can in this case define the rule firing strength for the fuzzy union operator by:

$$
\lambda_{i}=\min \mu_{\tilde{A} i j}\left(x_{j}\right) \quad j=1,2, \ldots m
$$

and alternatively, we can consider the fuzzy intersection operator and calculate the rule firing strength using:

$$
\lambda_{i}=\max \mu_{\tilde{A} i j}\left(x_{j}\right) \quad j=1,2, \ldots m
$$

These equations describe a single rule with single antecedent fuzzy system. In most practical cases, fuzzy systems are formed by a single rule with multiple antecedents or multiple rules with multiple antecedents.

Accordingly, the control algorithm is defined by the set of all fuzzy rules delineating the given problem. Each of these rules is denoted by an implication function (or implication operator represented usually as T-norm) that takes the input as the rule firing strength (as calculated by the above equations) and the resulting output as the fuzzy set for the command $z$.

The most commonly used implication function in fuzzy logic control is the Mamdani-type implication function $\min$ (Mamdani, 1975), which is given by: 


$$
\check{\mathrm{Z}}_{i}(z)=\min \left(\lambda_{i}, B_{i}\right)
$$

The case of a single proposition in the consequent was considered because rules with multiple propositions in the consequent are assumed to be separable into distinct rules.

The aggregation (employing usually an aggregate operator with T-conorm) is the process of aggregating qualified consequent membership functions or fuzzy sets from different rules to generate a single output fuzzy set, $\check{Z} \in Z$. Fuzzy operators like max can be used for this purpose:

$$
\check{Z}=\max \left(\check{z}_{i}(z)\right)
$$

To be more specific in identifying the fuzzy logic represented above, we refer to the parameterized inference method employing Equations (3-1-12) and (3-1-13) for aggregation and implication, respectively, as the max-min composition method. Though it may not be as "mathematically tractable" as the "max-product" composition, the Mamdani inference method is appropriate for inference systems used in fuzzy control and is widely used due to its minimal computational requirements. As has been noted earlier, different inference methods have been developed, such as the sum-prod method and the max-product composition. In general, the first nomination refers to the aggregation operator used, and the second notation is the implication operator. The above discussion summarizes the Mamdani-type fuzzy inference system. 
The final stage in fuzzy reasoning is the defuzzification process, or the defuzzification operation. By this, we mean the transformation of the "fuzzy" output of a fuzzy controller into a crisp single value output. This mathematical process can be computationally tedious. For the sake of computation efficiency it is generally performed using an averaging technique. The best-known methods assigned to this operator are: the centre of sums, bisector of area, centroid-of-area or centre-of-gravity, mean-of-maxima, smallest of maximum, largest of maximum (Jager, 1995). In this thesis the centroid-ofarea method is used, as described by the following relation.

For the output fuzzy set $\check{Z}$, with a discrete universe of possible crisp outputs $Z=$ $\left[z_{1}, z_{2}, \ldots, z_{M}\right]$, the result of the defuzzification process is computed as:

$$
C=\frac{\sum_{k=1}^{M} \mu_{\bar{Z}}\left(z_{k}\right) \cdot z_{k}}{\sum_{K=1}^{M} \mu_{\check{Z}}\left(z_{k}\right)}
$$

where $\mu \check{Z}\left(z_{\mathrm{K}}\right)$ is derived from:

$$
\mu_{\grave{z}}\left(z_{k}\right)=\max \mu_{z i}\left(z_{k}\right) \quad i=1,2, \ldots M
$$

A detailed example describing the fuzzy logic steps introduced earlier is included in Appendix A.

For Mamdani-type inference systems, defuzzification based on the centroid-ofarea method requires integration across the two-dimensional function. Integration operation is computationally demanding and can cause significant real-time delays, so the Matlab software used in this thesis implements a weighted average method for the 
centroid-of-area calculation to reduce the computational time. Figure 3-5 illustrates how the Mamdani-type FIS calculates the output variable from two input linguistic variables. The degree of overlapping of membership functions allows a high resolution $\mathrm{I} / \mathrm{O}$ function between crisp input and output variables translated in a smooth transition between operating points in control application. From Figure 3-5, the minimum of the two consecutive input values $(0.7$ and $0.5,0.4$ and 0.5$)$ is taken as the firing strength of the rule. In the example shown, the values 0.4 and 0.5 are the input membership values, $\mu$, aggregated at the output to give the calculated crisp value or centroid $C$.

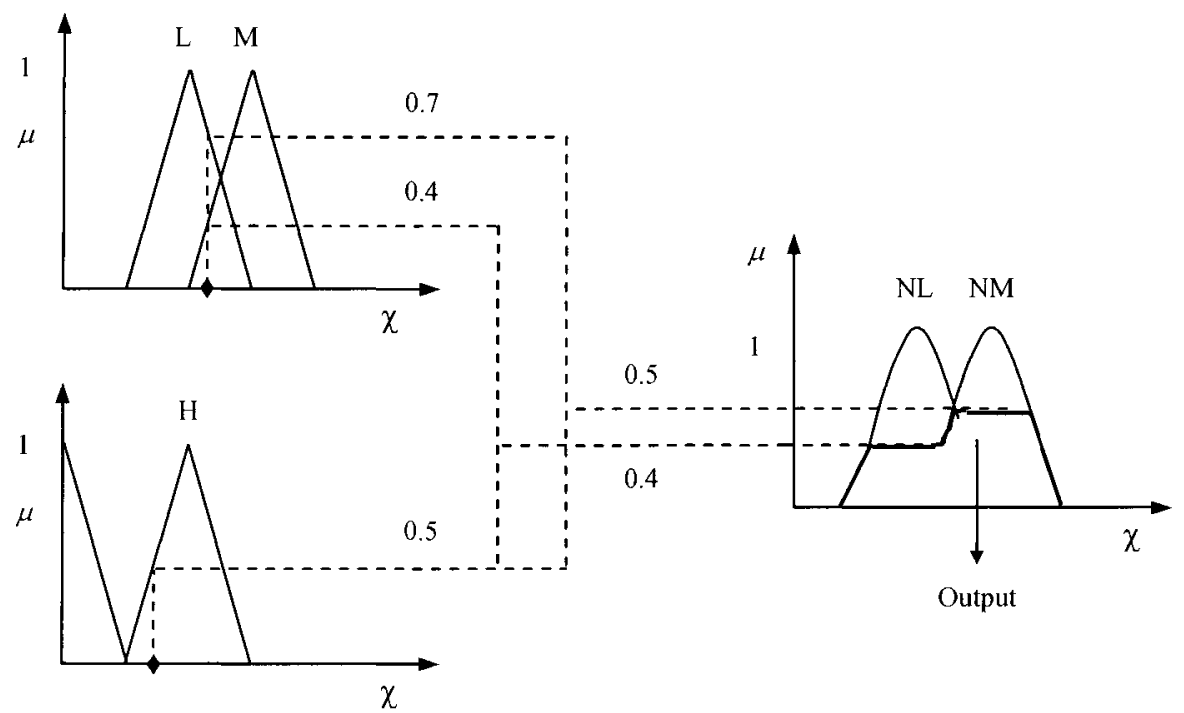

Figure 3-5: Rule calculation for a two-input single-output Mamdani FIS NL (negative large) and NM (negative medium) are fuzzy linguistic terms

Other types of inference systems are the Sugeno-type system and the Tsukemato fuzzy model. The Sugeno fuzzy model is discussed in the next section. 


\subsubsection{Sugeno fuzzy model}

A Sugeno fuzzy system with $M$ number of rules in the rule-base and two inputs and one output, is defined as follows:

$$
\text { If } x \text { is } \tilde{A}_{l, i} \text { and } \mathrm{y} \text { is } \tilde{A}_{2, i} \text { then } z_{i} \text { is } \zeta_{i}(x, y) \quad i=1,2, \ldots, M
$$

where $\tilde{A}_{l, i}$ and $\tilde{A}_{2, i}$ are fuzzy sets of the $i^{t h}$ in the antecedent with membership functions $\mu_{\tilde{A} 1, i}$ and $\mu_{\tilde{A} 2, i}$ respectively, and $\zeta_{i}(x y)$ is a crisp function of the model inputs in the consequent. For a zero-order Sugeno fuzzy system the consequent is defined as a constant:

$$
\zeta_{i}(x, y)=\text { const }
$$

This case is similar to the Mamdani-type inference system, having the consequent membership functions as singletons and an aggregation function including all the singletons. A particularity of the zero-order Sugeno inference model is its ability to produce a smooth function for the inputs given appropriate overlapping of the antecedent membership functions (Petriu, 2004).

A first-order Sugeno fuzzy model produces consequents as a polynomial of the form:

$$
\zeta_{i}(x y)=p_{i} \cdot(x)+q_{i} \cdot(y)+r_{i}
$$

with each rule infering a crisp output, the weighted average as calculated below is used in the inference engine for defuzzification (Takagi and Sugeno, 1985): 


$$
\zeta=\frac{\sum_{k=1}^{M} \mu_{z}\left(z_{k}\right) \cdot \zeta_{i}}{\sum_{k=1}^{M} \mu_{\bar{z}}\left(z_{k}\right)}
$$

Where $\mu_{\bar{z}}\left(z_{k}\right)$ is found in Equation 3-1-15, and $\zeta_{i}$ is the consequent parametrized as a linear or constant function of the inputs. Figure 3-6 shows a smooth linear approximation of a function using a Sugeno fuzzy system with three rules.
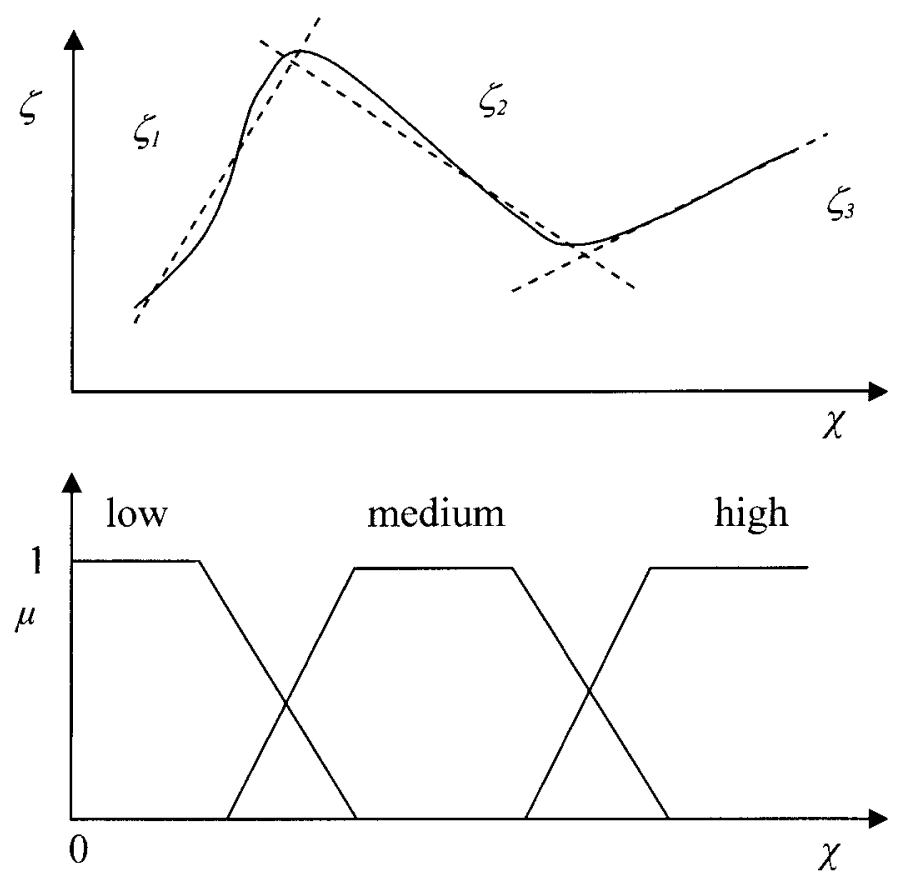

Figure 3-6: Linear approximation using Sugeno fuzzy model (adapted from Babuska, 1998)

This type of fuzzy rule-base model is very suitable for nonlinear function approximation and its application in function mapping will be discussed in Chapter 5 . 


\subsection{Advantages of fuzzy logic}

Fuzzy logic is a useful tool because the set of fuzzy rules are described using common language, and knowledge is drawn from human experience and knowledge of the dynamic behaviour of the system in question (through approximate reasoning using an intuitive approach). This approach allows the developer to deal with the uncertainty and nonlinear nature of dynamic systems without developing overly complex mathematical models. In addition, fuzzy systems are easy to implement and maintain using software on existing microprocessors or hardware (Khan 1999).

\subsection{Disadvantages of fuzzy logic}

As the set of rules increases, it becomes more difficult for the user to follow them, and to tune membership functions to obtain good results. In fact, the number of rules required to form a complete rule-base increases exponentially with the number of linguistic terms. In practice, 15 rules for every application is deemed easy to follow, and rule-base with higher numbers of rules are harder to track by the developer (Chen, 1999). In this case, adaptable fuzzy approaches, neurofuzzy and genetic algorithms (GA) can all be useful techniques in defining and fine tuning the rule-base.

Another disadvantage is that fuzzy logic uses fixed geometric-shaped membership functions requiring more system memory and processing time. It also relies on heuristic algorithms for defuzzification, rule evaluation, and antecedent processing. Heuristics do not guarantee satisfactory solutions under all possible conditions nor allow adequate generalization capabilities to deal with unforeseen situations. 
Another downside is the inability of fuzzy systems to learn (i.e., to learn from experience) since the rules are predetermined (except in adaptive fuzzy systems, which allow limited flexibility). Accuracy is improved only through trial and error.

The next chapter will introduce the artificial neural network, another artificial intelligence technique. 


\section{Chapter 4}

\section{Artificial neural networks}

\subsection{Introduction to artificial neural networks}

A general definition of artificial neural networks (ANNs) is found in Simpson (Hecht-Nielson, 1990):

A neural network is a parallel, distributed information processing structure consisting of processing elements (which can possess a local memory and carry out localized information processing operations) interconnected together with unidirectional signal channels called connections. Each processing element has a single output connection which branches (fans out) into as many collateral connections as desired (each carrying the same signal-the processing element output signal). The processing element output signal can be any mathematical type desired. All of the processing that goes on within each processing element must be completely local; i.e., it must depend upon the current values of the input signal arriving at the processing element via impinging connections and upon values stored in the processing element's local memory.

Or in a more simplified form, "an ANN is a nonlinear directed graph with edges that is able to store patterns by changing the edge weights and is able to recall patterns from incomplete and unknown inputs" (Simpson, 1990).

Artificial neural networks, a synonym of connectionist learning and parallel distributed processing, are being expounded as a practical tool in major scientific areas: 
engineering, natural and cognitive sciences, finance, arts, music sound and pattern recognition, etc. In engineering applications, ANNs were investigated widely in modelling or system identification from data and control of nonlinear dynamic systems (Chiras et al., 2002) and (Fortuna et al., 1995).

\subsubsection{Evolution of artificial neural networks}

Dating back as early as 1940, ANNs were thought to provide computational solutions to complex problems through a simple process (independent from mathematical modelling). The structure of the artificial neural network was conceived as a basic technological imitation of the human brain, in particular that of the neurons (brain cells) and their connections (Figure 4-1). Although the computing ability of the ANN architecture depends on the computing tools (i.e. the computer), this parallel computing technique evolved progressively but within a limited horizon throughout the following two decades.

The 1950s and 1960s saw the introduction of two important paradigms: the perceptron and the ADALINE (ADAptive Linear Neuron) / Madaline (two or three layers intended for adaptive control, noise cancellation, and pattern analysis). As the artificial neural network and its applications became larger and more complex, different paradigms emerged. Starting in the 1980s, modern approaches such as backpropagation, competitive learning, and Hopfield networks in particular have revived interest amongst researchers in neural networks. Though these later networks still encompass some of the basic structural aspects of earlier ANNs, including the weighted sum, weight adjustment, 
thresholding, and supervised learning for pattern classification, Hopfield's 1982 breakthrough paper (Hopfield, 1982) demonstrated clearly the computational capabilities of a network with associative memory in solving optimization problems (Dayhoff, 1990).

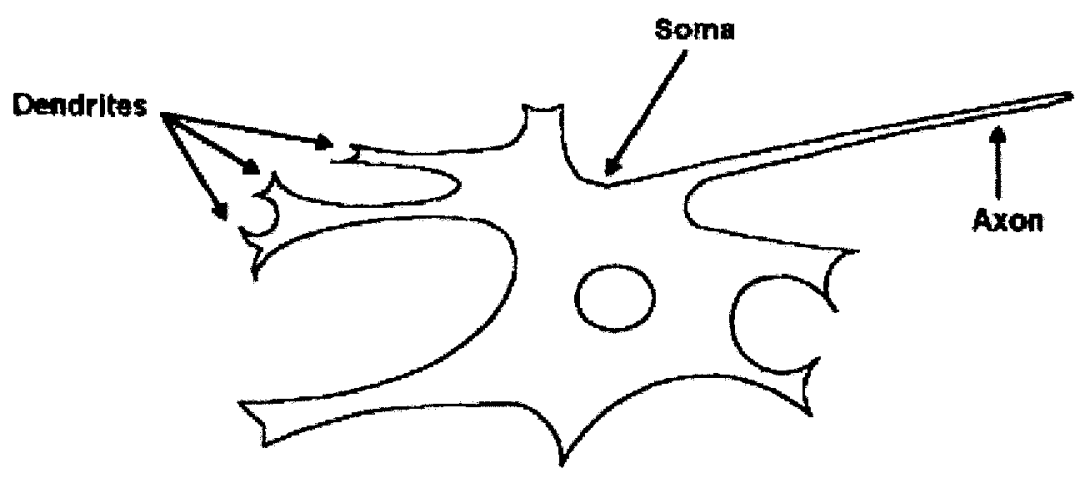

(a)

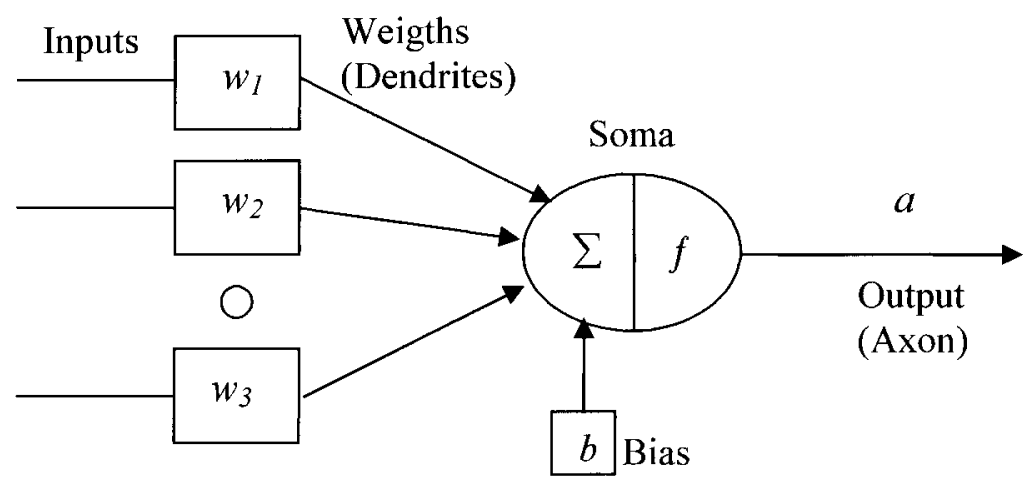

(b)

Figure 4-1: (a) Biological neuron, (b) Artificial neuron model (adapted from Balakrishnan, 1996) 


\subsection{Brief description of neurons}

As discussed earlier, there are two networks mentioned in this thesis; the biological network and the artificial network. The abbreviation ANNs refers to the artificial neural networks. The neural network is a combination of interconnected neurons. These neurons can be of a biological nature or can have a mathematical representation as will be explained later.

\section{Biological neurons}

Neurons are nerve cells that transmit nerve signals to and from the brain at a speed of up to $200 \mathrm{mph}$. A synthesis, according to functionality, of a biological neuron shows four major constituents: dendrites, which carry the electro-chemical signals into the neuron body from neighbouring connected neurons; the neuron body (soma) that carries the integrated incoming signals and contains the axon hillock; the axon, which transmits the neuron firing signal, if the aggregate inputs from all dendrites is larger then the axon hillock threshold; and the presynaptic terminal boutons, which carry signals to neighbouring neurons (West, 1985).

There are 100 billion neurons in the brain, varying in diameter from 4 to 100 microns. A typical neuron has 1,000 to 10,000 synapses allowing communication with 1,000 to 10,000 other neurons, glands, muscular cells, etc. (West, 1985).

\section{Artificial neurons}

The concept of the artificial neuron is derived from the notion of the biological neuron. Dating back as early as 1943, theories on neural computing were introduced 
(McCulloch, 1943). Figure 4-2 depicts the artificial neuron foreseen by McCulloch and Pitts (hereafter referred to as McC-P). Since this early emulation of the biological neural network, computing abilities have evolved from single perceptron model (Rosenbalt, 1958), which is composed of a neuron with a hard limit transfer function and weight adjustment mechanism, to the more complex and applicable neural networks (Kohen, 1972), which have renewed interest in the field of ANNs and recurrent networks (Hopfield, 1982) (based on Hebb’s rule).

The basic neural computations are the perceptron (suited for pattern recognition) and the ADALINE, which have a linear activation function with weights and biases adjusted according to the error magnitude using the Least Mean-Square (LMS) method (Widrow-Hoff learning rule). Both differ in their learning method but suffer from the same limitation: they can only solve linearly separable problems.

Figure 4-1(a) details artificial neuron synthesis. With analogy to the biological neuron, the lines shown in Figure 4-1(a) are the "axons", the "synapses" are the weights and the "soma" is the neuron body comprised of two segments: the first segment sums up the weighted outputs $(w p+b)$ of connected neurons. The second segment embeds an activation function (hard limit, linear, etc.). The activation function, $f()$, determines the activation level of the given neuron based on the weighted outputs $[f(w p+b)]$. 


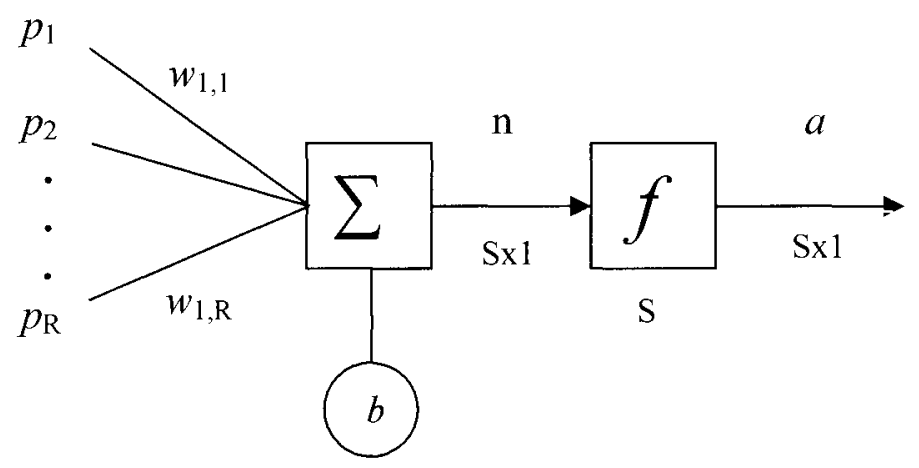

Figure 4-2: Neuron structure: $a=f(w p+b)$ (Matlab, 2000)

In the mathematical representation of the neuron, $a=f(w p+b), p$ denotes the input vector with magnitude $\mathrm{Rx} 1, b$ the bias, and $a$ is the $\mathrm{Sx} 1$ number of neurons in layer 1 , and net input $\mathrm{n}$ to the activation function is given by $\mathrm{n}=\sum w p+b$ or $w_{1,1} p_{1}+\ldots w_{1, \mathrm{R}} p_{\mathrm{R}}+b$.

This single neuron with a linear threshold function was originally perceived by Rosenblatt as the basis for the single layer neural network in an attempt to copy the endogenous discriminative ability of human thinking in the field of pattern recognition. However, these early networks were bounded by their inabilities to separate nonlinear problems. Figure 4-3 shows an McC-P neuron with binary threshold or a perceptron. 


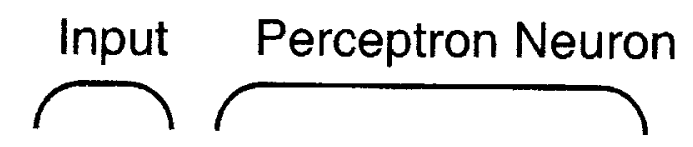

\section{Binary Threshold}
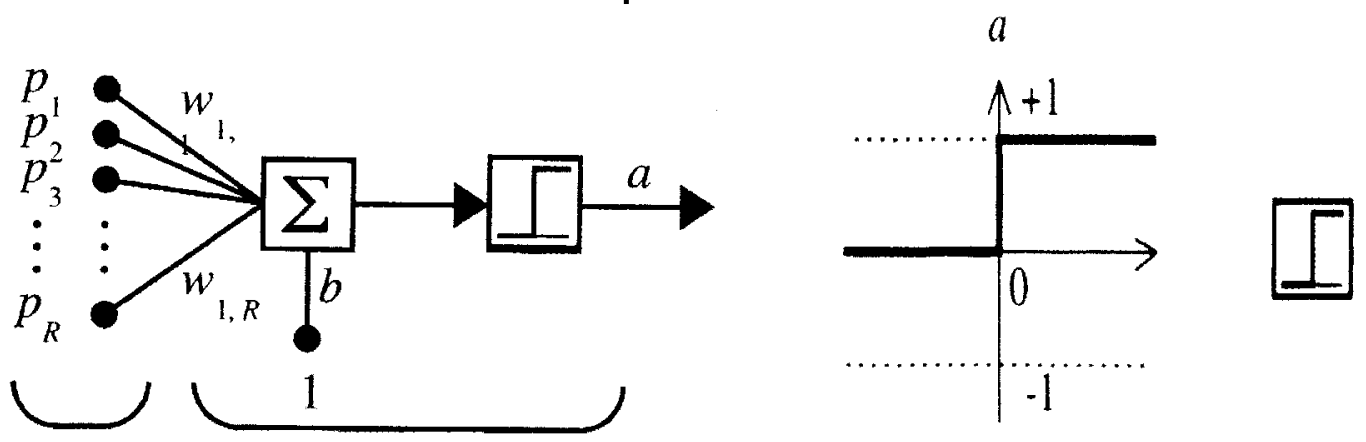

Figure 4-3: Perceptron with binary threshold (Matlab, 2000)

\subsection{Artificial neural networks}

An artificial neural network can be viewed a cluster of neurons, arranged in layers, connected by "weights" with firing strength to activate a function. The weights are usually in scalar form and are adjusted during the training or learning phase. The basic application of ANN is to "map" an input vector to a corresponding output vector. The topology (also known as configuration or anatomy) of the network varies with the mapping environment for which it is used. Authors have proposed different network topologies for various applications, some of which are: Feedforward, LevenbergMarquardt, Radial-Basis-Function (RBF), Backpropagation network (BP), Dynamic Neural Unit (Rao, and Gupta, 1992), Cerebellar model articulation controller (CMACAlbus, 1975), Autoassociative, Wavelet, and the Stacked Network (Wolpert, 1992). All these networks fall under two generic categories: recurrent and non-recurrent. The distinction between these two groupings is made at the level of neural connection and 
weight selection. Recurrent networks (Hopfield, 1982) have internal feedback paths (cycles) making them dynamic networks. In contrast, the non-recurrent topology exhibits no internal cycle connections and can be seen as a transformation of n-dimensional Euclidean space to m-dimensional Euclidean space (Hornik, 1989).

Figure 4-4 illustrates the network topology of two widely known ANNs, the feedforward non-recurrent ANN (Figure 4-4(a)) and the fully recurrent ANN (Figure 44(b)).

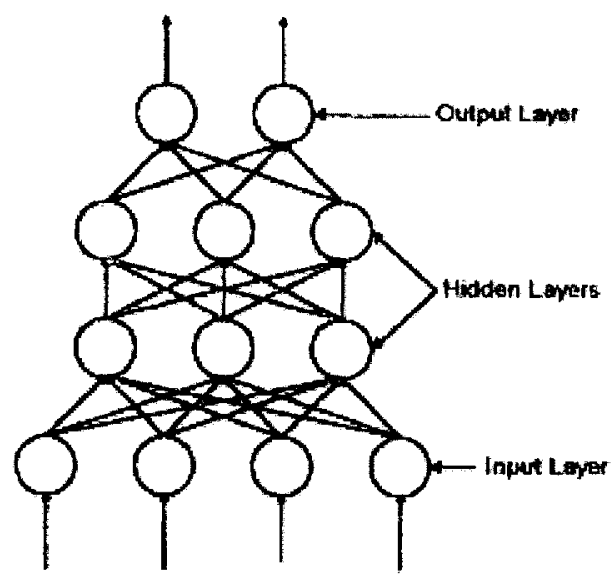

(a)

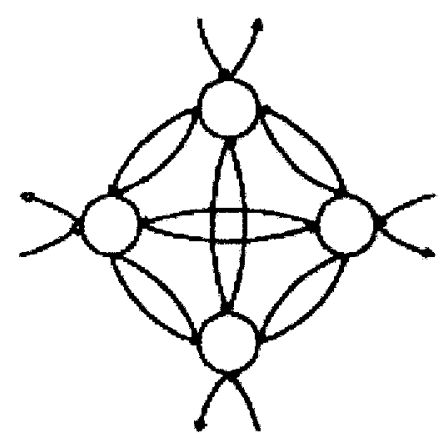

(b)

Figure 4-4: (a) Feedforward non-recurrent ANN; (b) Fully recurrent ANN

The general representation of the recurrent neural network shown in Figure 4-4(b) can be extended to the particular and more detailed neural network depiction in Figure 45. 


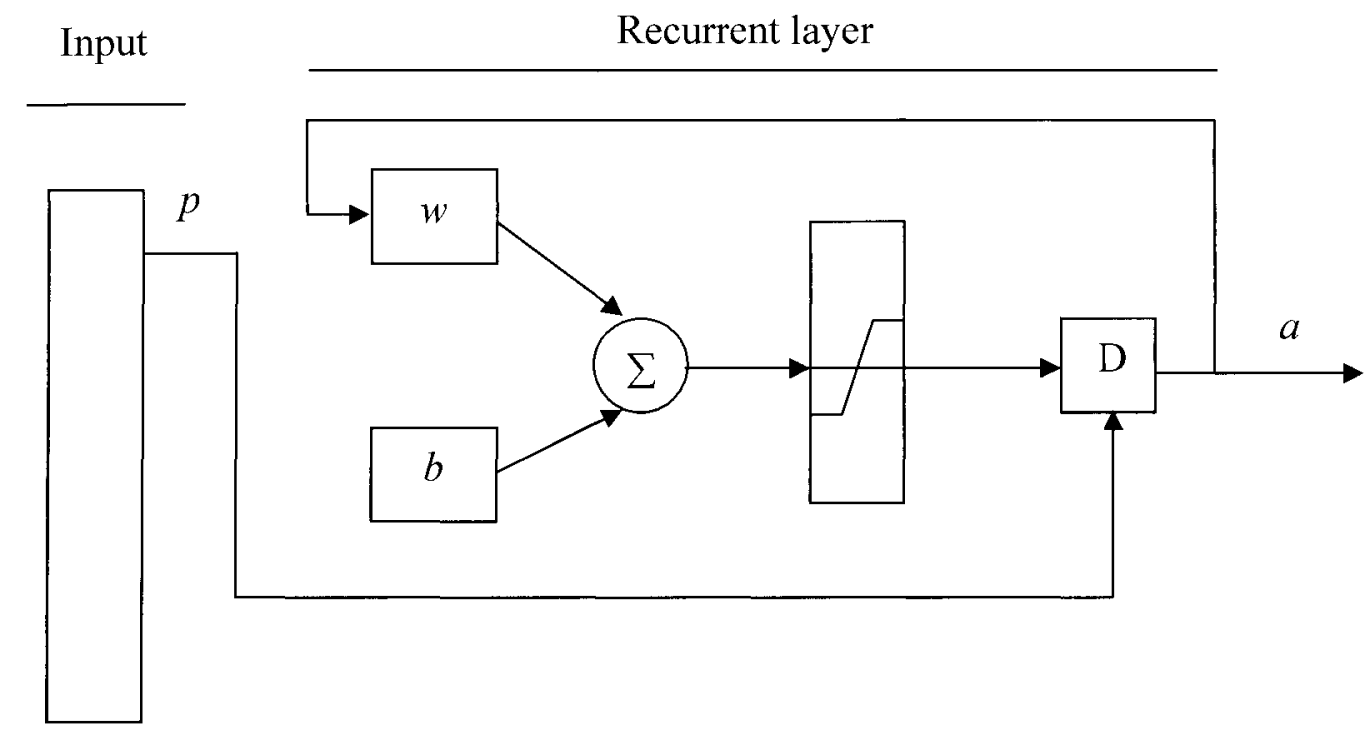

Figure 4-5: Recurrent artificial neural network topology where D denotes the time delay in the Matlab representation(adapted from Matlab, 2000)

\subsubsection{Architecture of ANNs}

Artificial neural networks are formed with multiple interconnected processing units (neurons), structured in several layers primed with the ability to learn system behaviour and generalize from training data (Kaur and Lin, 1998). It is exemplified by the number of input and output nodes, the number of layers (including hidden layers), the number of neurons in each layer, the interconnection scheme of layers, and the transfer/activation function selected for each layer. Figure 4-6 schematizes a two-layer feedforward neural network with multiple neurons in the first layer (sigmoid activation function), multiple neurons in the second layer (linear activation function), and one input vector of $Q$ size. The number of hidden layers depends on the complexity of the problem represented by the ANNs. 


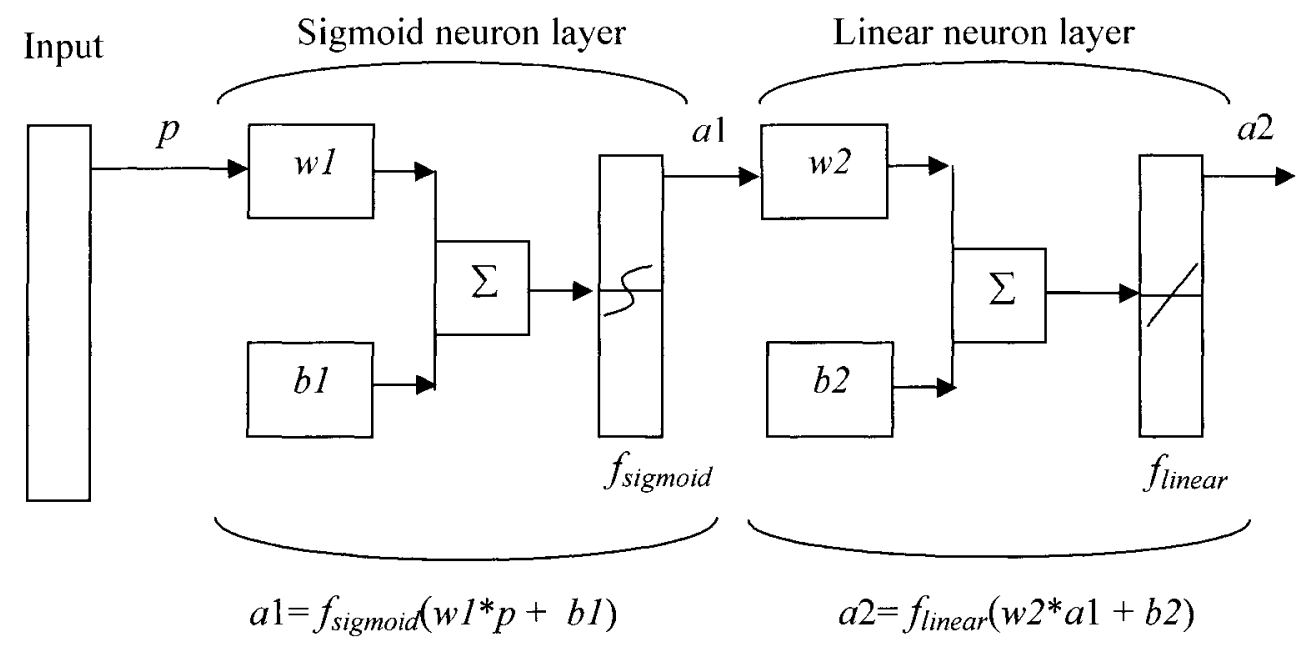

Figure 4-6: Two-layer ANN with sigmoid and linear activation functions (adapted from Matlab, 2000)

\subsubsection{Training of ANNs}

Training methods of ANNs can be divided into two main categories: supervised learning and unsupervised learning. Both methods differ in the way the data is provided to the ANN. In the supervised method, the network is exposed to input vector data and its corresponding output vector data, referred to as a training set. The error between the desired and the actual output is to be minimized. The ANN weights are initialized randomly and then are adjusted according to a learning algorithm to achieve, after several iterative steps, the desired match between the input and output set of data. Once the goal is met, the weights are fixed and the network is ready to generalize (i.e. predict an output from a given input vector that differs from the training input vector). Supervised learning 
can be executed off-line and may be time-consuming depending on the size and type of the training data. The second training technique is the unsupervised learning or selfsupervised learning (Nelson et al., 1991). As the name suggests, unsupervised learning needs no external performance monitoring, but rather its operation is evaluated by an internal index. Under this assumption, the learning algorithm highlights persistent patterns in the input vector and makes adaptations to deduce the output according to the application for which the network is structured. The unsupervised paradigms suggested in the literature comprise the Kohen self-organizing features map and the competitive learning. Once the training mode is established, the training algorithm must be selected.

\subsubsection{ANNs learning algorithm}

After the ANN is defined and structured, a training algorithm is selected to allow the network, by updating its weights and biases, to match a set of input data to their corresponding output. During training the weights and biases are adjusted to minimize a predefined network performance function. This performance function could be the average error between the network outputs and the target outputs known as the mean square error (Matlab, 2000). In the case of feedforward networks, different training algorithms that use the gradient of the performance function to adjust the weights and biases were developed. Two of these methods are discussed in this section: the backpropagation and the Levenberg-Marquardt training algorithms. 


\subsubsection{Backpropagation method}

Presented as the general delta rule, the backpropagation method (Figure 4-7) is probably the most common learning algorithm employed in the training of multiplayer ANNs. It is formulated as an iterative, 'steepest descent' algorithm with the mean square error between a desired and an actual output response as the performance index (Rumelhart, 1986).

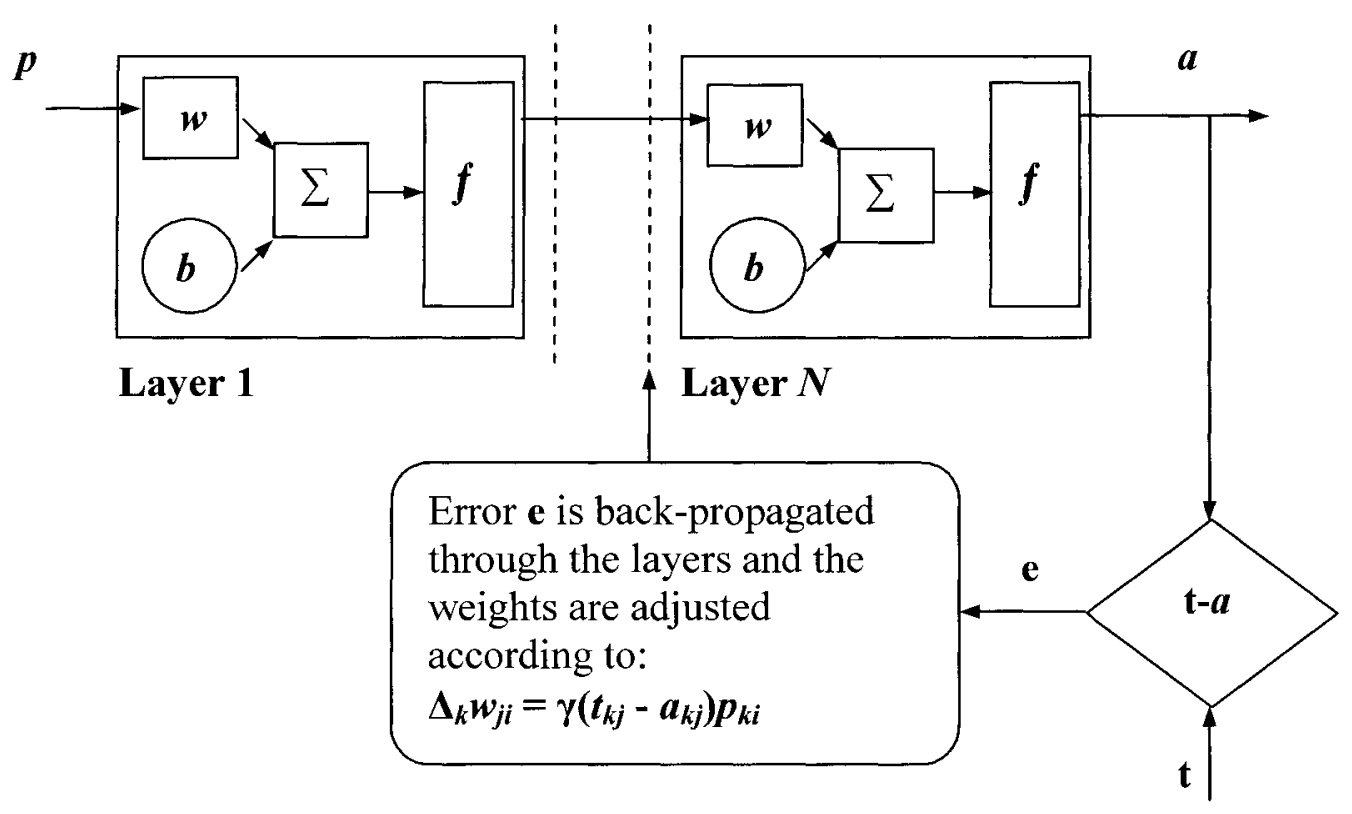

Figure 4-7: Backpropagation learning algorithm

With this technique, a set of paired input vectors and corresponding output vectors, known as target vectors, are introduced to the ANN. The network then produces its own output vector or pattern from the input vector and compares the result with the target output, and an error is generated. For each iteration $i$, the weights, originally initialized randomly (or in some cases preset), are adjusted to minimize the induced error (target - actual output) according to the following rule: 


$$
\Delta_{k} w_{j i}=\gamma\left(t_{k j}-a_{k j}\right) p_{k i}
$$

Where $t_{k j}$ represents the target output for the $j^{\text {th }}$ element of the output vector $k, a_{k j}$ is the $j^{\text {th }}$ element of the actual output vector corresponding to the input training vector $k, p_{k i}$ epitomizes the $i^{\text {th }}$ value of the input vector, $\gamma$ is the learning rate, and $\Delta_{k} w_{j i}$ is the calculated weight or connection strength adjustment for the $i^{\text {th }}$ to $j^{\text {th }}$ neurons. Though its concept looks straightforward, backpropagation suffers from significant disadvantages making some of its applications limited. First, it requires an extensive number of iterations for matching/learning the pattern. Second, it is computationally demanding since the whole training set/vectors must be propagated through the network to calculate the gradient of the error (i.e. the computation time per iteration varies in proportion with the number of layers and units in each layer), making it impractical for large networks with on-line training. For example, for feature recognition exercises, it takes 4,123 epochs and 2591 seconds to train a network with backpropagation method as compared to only 5 epochs and 1.9 seconds to train an RBF network (Petriu, 2004). Third, the results of the training and the upgrading of the weights of the network after each iteration depend heavily on the selection of the parameter or "learning rate" $(\gamma)$. Finally, with this method, a pattern is considered correctly classified if the discrepancy between the output vector and the target vector is smaller than any other output vector. Furthermore, this approach does not allow the network to avoid the local minima to which the gradientbased method is susceptible. 


\subsubsection{Levenberg-Marquardt training method}

This training method is based on standard numerical optimization techniques to calculate the second-order gradient of the steepest descent method without computing the Hessian matrix; it uses just an approximation of that matrix (Equation 4-3-2). The advantage of such methodology is a gain in training speed. The Lavenberg-Marquardt algorithm can be represented as follows:

$$
w_{k+l}=w_{k}-\left[\boldsymbol{J}^{T} \boldsymbol{J}+\theta \boldsymbol{I}\right]^{-1} \boldsymbol{J}^{T} e
$$

where

$I$ is the identity matrix,

$\boldsymbol{J}$ is the Jacobian matrix of the form:

$$
\left[\frac{\delta e^{2}(k)}{\delta w_{1}(k)} \quad \frac{\delta e^{2}(k)}{\delta w_{2}(k)} \cdots \frac{\delta e^{2}(k)}{\delta w_{N}(k)}, \frac{\delta e^{2}(k)}{\delta b(k)}\right]
$$

where $e$ is the vector of network error, and $\theta$ is a scalar which is reduced successively with the performance function after each iterative step. This algorithm is the fastest training method for moderate-sized neural feedforward networks (of approximately several hundred weights). It can be efficiently implemented in Matlab, "since the solution matrix equation is a built-in function" (Matlab, 2000). 


\subsection{Types of artificial neural networks}

ANNs can be classified by their structures (number of layers, number of neurons/layer), topology (neuron connectivity, recurrent, non-recurrent), training methods (supervised, unsupervised) and learning algorithms. In this thesis, two networks will be presented; the multilayer perceptron and the feedforward neural network.

\subsubsection{Multilayer perceptron}

The multilayer perceptron (MLP) can be viewed as an array of neurons arranged in layers with each input to a neuron, in any layer, making a linear weighted combination of the outputs of all the neurons in the precedent layer. The output signal from the neuron results from an activation function according Figure 4-6:

$$
p_{i}=M_{i}\left(p_{i-1}\right)=\Gamma\left(w_{i} p_{i-1}+b_{i}\right)
$$

where

$p_{i} \quad$ is the vector of the outputs of the neurons in the $i$ th layer;

$w_{i} \quad$ is the $(i-1)$ th to $i$ th layer connections weights matrix;

$b_{i} \quad$ characterizes the bias vector of the neurons in the $i$ th layer; and,

$\Gamma()$ is a vector-value function defined as

$$
\Gamma\left(\begin{array}{l}
\chi_{1} \\
\chi_{2} \\
\chi_{n}
\end{array}\right)=\left(\begin{array}{c}
f\left(\chi_{1}\right) \\
f\left(\chi_{2}\right) \\
f\left(\chi_{n}\right)
\end{array}\right)
$$


The term $f()$ is a linear or nonlinear activation function. Examples of activation functions are the sigmoidal activation function and the S-shaped function known as the squashing function:

$$
\begin{aligned}
& f_{\text {sigmoid }}(\chi)=\frac{1}{1+\mathrm{e}^{-\chi}} \\
& f_{\text {s-shaped }}(\chi)=\frac{\mathrm{e}^{\chi}-1}{\mathrm{e}^{\chi}+1}
\end{aligned}
$$

It is worth mentioning that the most common activation functions for system identification are the sigmoid function (Equation 4-4-3(a)) and the hyperbolic tangent function (tanh) shown in Figures 4-8 and 4-9. The hyperbolic tangent function is given by

$$
f_{\text {tanh }}(\chi)=2 f_{\text {sigmoid }}(\chi)-1
$$

As can be noticed, the sigmoid and tanh functions are both nonlinear and are closely related so that it does not make great difference as to which one should be chosen to represent a nonlinear process. However, the nature of the ANN is defined by the choice of the form of the activation function (Cybenco, 1989). 


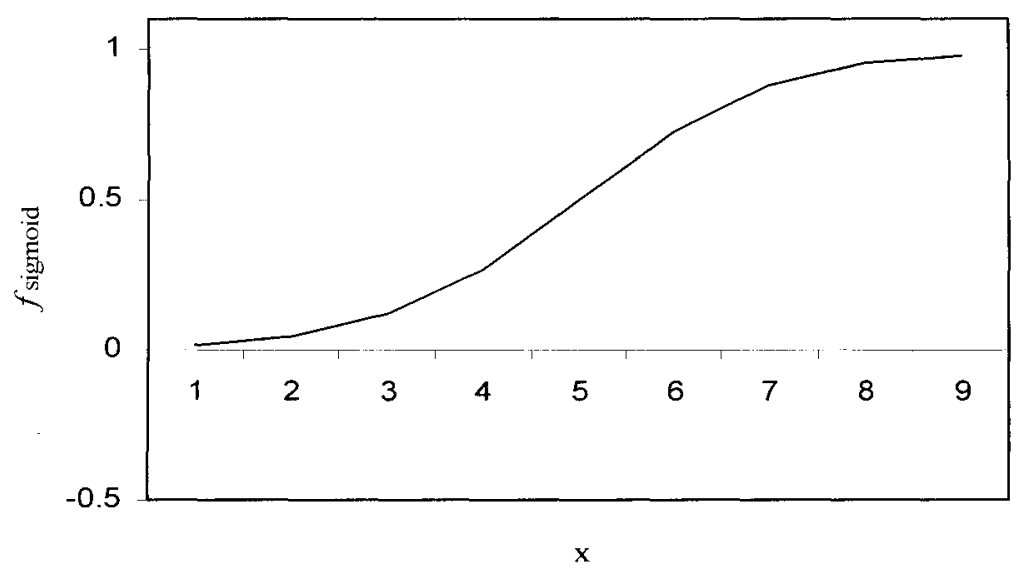

Figure 4-8: Sigmoid activation function

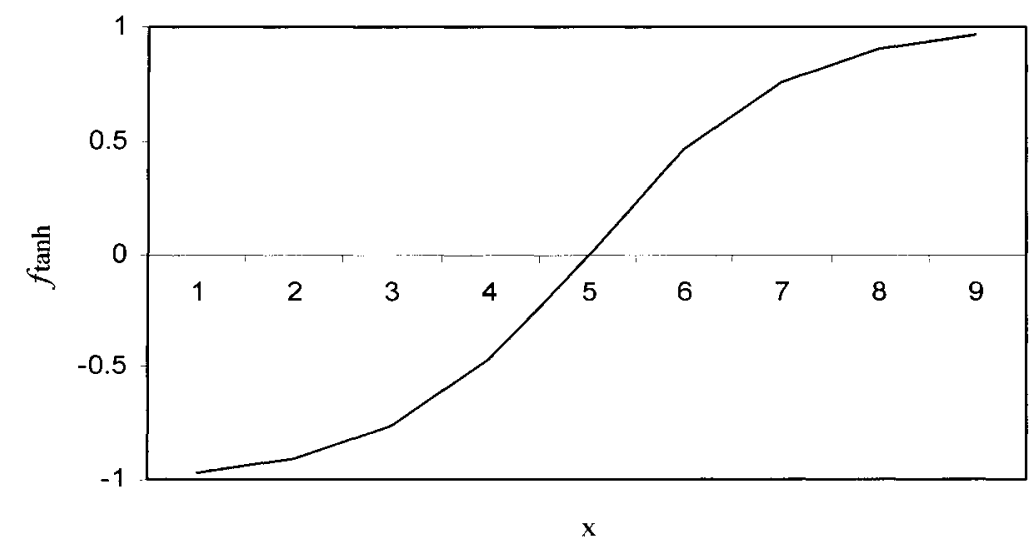

Figure 4-9: Tanh activation function

\subsubsection{Feedforward artificial neural networks}

This network consists of one or more hidden layers of sigmoid neurons and an output layer of linear neurons. The neurons with nonlinear activation functions allow the basic feedforward network to perform a nonlinear transformation of input data in order to approximate the output data (Matlab, 2000). Figure 4-10 shows a two-layer feedforward 
network. The number of input and output nodes is determined by the nature of the modelling problem being tackled, the input data representation and the form of the network output required. The number of hidden layer nodes is related to the complexity of the system being modelled. The interconnections within the network are such that every neuron in each layer is connected to every neuron in the adjacent layers. Each interconnection has associated with it a scalar weight that is adjusted during the training phase. The hidden layer nodes typically have sigmoidal transfer functions. The activation functions and the learning rules define the structure of the ANNs, and both depend on the real nature of the application for which the network is designed. The capabilities of feedforward networks to approximate any continuous function were emphasized by Cybenco (1989). 
Input

Layer 1

Layer 2

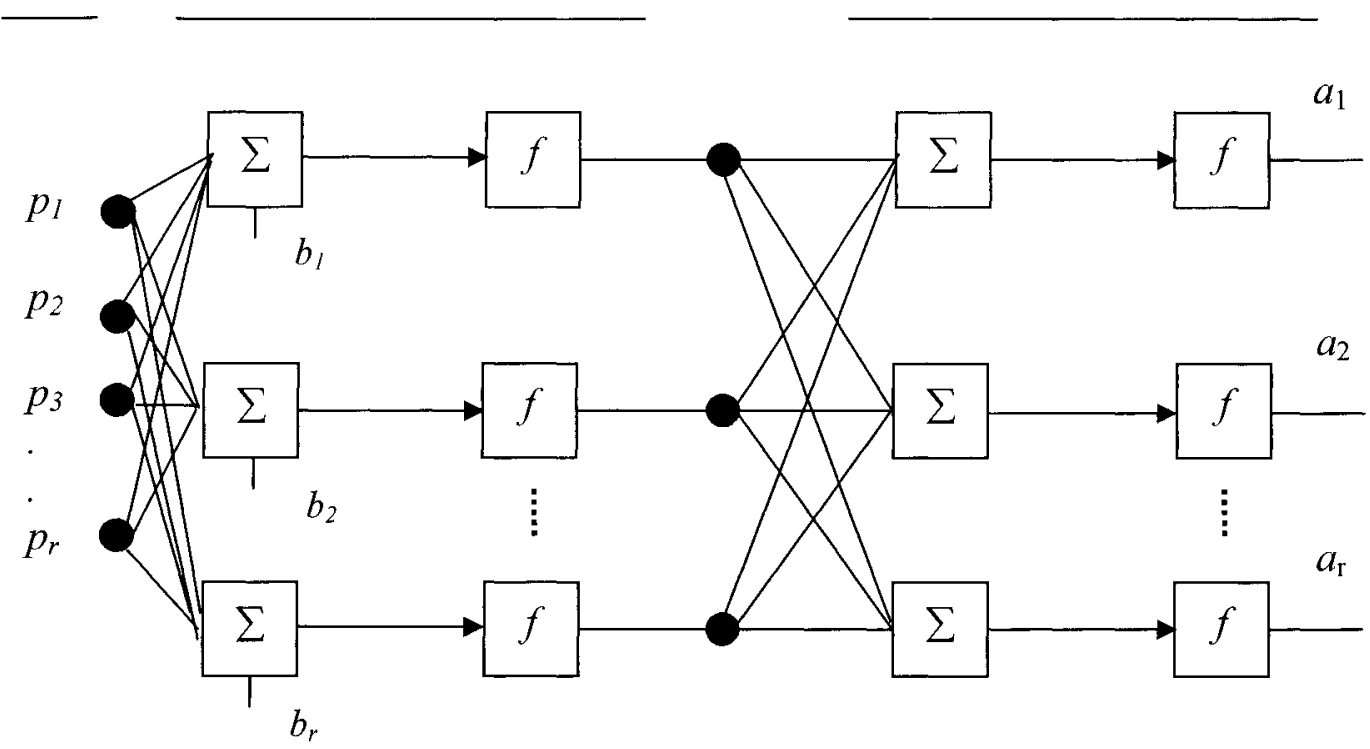

Figure 4-10: Two-layer feedforward artificial neural network: $p$ 's are the input vectors, $b$ 's are the biases, and $a$ 's are the output vector (adapted form Matlab, 2000)

\subsection{Advantages of artificial neural networks}

ANNs do not need prior knowledge of a mathematical model, but are characterized by learning algorithms that adjust consecutively by trial and error during the learning phase the weights and biases of the neurons. These learning and generalizing capabilities permit the network to tackle more effectively nonlinear time variant problems, and to provide solutions to either unsolved or poorly addressed problems by other available techniques, including fuzzy logic. An added advantage of ANNs is their capacity to develop solutions to meet a pre-specified accuracy (Khan, 1999). 


\subsection{Disadvantages of artificial neural networks}

It is unclear how ANNs learn the input output relationships and map them to their weights. It is also difficult to determine the proper size and structure (i.e. how many neurons and how many layers) of the network that will effectively address the given problem.

Since ANNs do not scale well, manipulating learning parameters (weights, bias, activation level unit) for supervised learning and convergence is tediously complex. Another drawback is the increased cost of implementation (due to the large memory and computing time required) of software and hardware based on an ANN (especially for control applications) as compared to other available technologies such as fuzzy logic systems, which are less complex and require shorter processing time.

\subsection{Modelling with artificial neural networks}

Modern gas turbines epitomize a significant nonlinear control application area because the performance and efficiency of such engines can be improved greatly with a well-formulated fuel flow schedule. The resulting enhanced performance, in terms of compressor stall margins and turbine temperature limits, was sought during recent years using the Linear Quadratic Gaussian/Loop Transfer Recovery (LQG/LTR). Such a method is used in multivariable controller designs. On the other hand, the Linear Quadratic Gaussian/Linear Quadratic Regulator (LQG/LQR) technique provides stability robustness, with noise rejection for minimum phase systems, but it does not have the properties to cope with nonlinearities caused by changes in the engine operating 
conditions. These variations include changes in the ambient conditions, altitude speed and modelling errors. Song (1994) introduced an adaptive controller that tolerates structured uncertainty for gas turbine engines. Nonetheless, it is limited by the linear parameter estimation method that has partial performance improvement. To overcome this limitation, new methods based on artificial neural networks (Song, 1994) and fuzzy logic (Fortuna et al., 1995) were considered.

Improved learning algorithms, in conjunction with innovations in microcomputing and electronics, have enthused researchers' interest in ANNs for modelling and control of nonlinear dynamic systems. In each of these two fields of interest, ANNs have showed some attractive properties. In the control field for instance, ANNs offered the ability to nonlinear plant modelling and multi-sensory information, the execution of collective processing and learning (both on the supervised and nonsupervised training level), and the potential for parallel computing (Wu et al., 1992).

The application of ANNs in the modelling of gas turbine engines will be covered in detail in the following chapter. 


\section{Chapter 5}

\section{Simulation analysis}

This chapter will summarize the transient operation phase of a gas turbine engine, since this is the most critical phase, as mentioned in Chapter 2. A fuzzy logic controller will be proposed to provide an open-loop control for the gas turbine engine during acceleration and deceleration. An artificial neural network will be built to model the performance of the mentioned engine during transient operations. The controller will relate the gas generator speed $\left(\mathrm{N}_{\mathrm{GG}}\right)$ to the fuel ratio and the ANN will generate the relationships between the $\mathrm{N}_{\mathrm{GG}}$ and the fuel flow, and between the TIT and the fuel flow. These relations are important factors in the control of the gas turbine engine.

This chapter is divided into two parts. Part one (sections 5.1-5.3) presents the fuzzy logic controller design. Part two (sections 5.4 and 5.5) discusses the artificial neural network modelling approach.

\subsection{Fuzzy logic controller}

Fuzzy logic controller is a nonlinear methodology that converts linguistic control strategies, based on expert knowledge, into a qualitative and accurate control scheme. The structure of the controller is well defined by the if-then relation within the rule-base. The rule-base allows the mapping of input vectors to a corresponding output action vector. With proper membership functions overlapping, the adequate selection of 
fuzzification processes and the firing strength of each rule, FLC can precisely match any control strategy. The controller input could be the error and rate of change of the error to account for the nonlinearity in the systems dynamics. The following two sections will demonstrate the application of the FLC to the control of GTE. Before detailing the control strategy, an introduction to the software used in this thesis and some definitions related to the performance of the GTE will be made.

\subsubsection{Performance characteristics of GTE}

The GTE is defined by the thermodynamic cycle relations between different variables (pressure, speed, power, efficiency, etc.). Various cycles are tested depending on the application of the GTE before a final design or geometry is selected. This final design (set at a desired operating condition of speed and power) or the design point (DP) defines the overall configuration of the GTE (i.e., the aerodynamic and thermodynamic aspects). These include the total mass flow rates, pressures, temperatures at inlet and exit conditions, and efficiencies. The performance of the GTE is then evaluated at different operating conditions (or at off-design points (OD)) and the individual components (compressor, combustor, turbine, etc.) are selected and designed. These points can be seen in the characteristic maps of every component. These maps include lines such as the surge line (SL), choke line (CL), and operating line (OL) (a series of off-design points). Figure 5-1 illustrates the DP on a mass flow versus pressure ratio graph. 


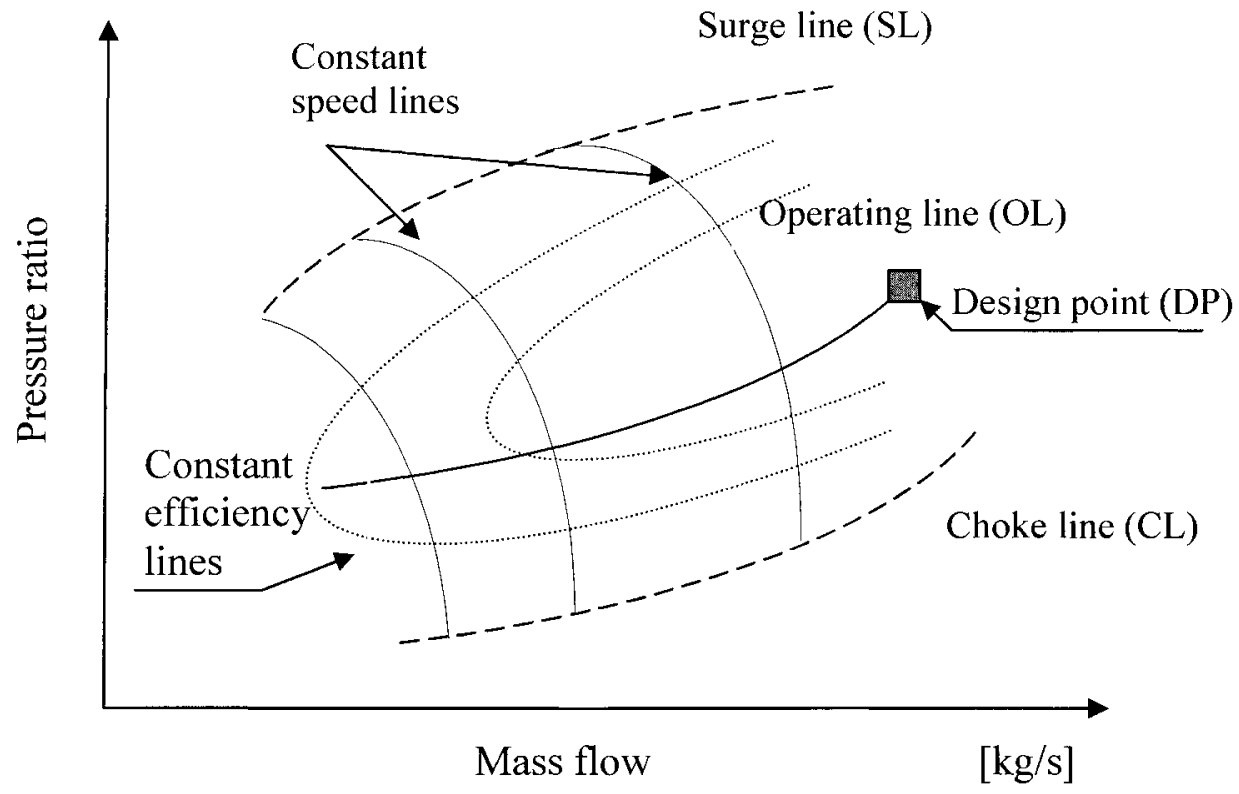

Figure 5-1: Schematic of a generic compressor map with DP shown

The final step is to assess the transient behaviour of the GTE by adding the polar moment of inertia and action of the controller. The design steps mentioned above will be described as follows:

Design point

When a GTE is designed, different thermodynamic cycles are assessed for the particular intended application of the engine. Specifically, the thermodynamic relations between different components are evaluated and determined. These include the pressure ratio, mass flow rate, total temperature, pressure losses and overall efficiency for a given operating speed. The most feasible design data which offer the highest overall efficiency 
for the desired GTE power are selected and termed the design point (Cohen et al., 1987). The individual components are then designed to meet the overall efficiency criterion and their performance characteristics are determined. This is referred to in the literature as the off-design point determination.

\section{Off-design points}

When the different GTE components are linked together to form the engine their operating range is reduced Cohen et al., 1987). However, the performance of the GTE must be determined at different operating conditions (i.e., the conditions that differ from that of the design point), and this can be done by applying the compatibility of flow, speed, and work between different GTE components. By doing that, the off-design point selected on the compressor map and its corresponding off-design point shown on the turbine map will be obtained. In GasTurb software, the determination of each off-design point requires iterations where different thermodynamic input variables are estimated. For this purpose the Newton-Raphson iteration technique is used. This error-minimizing technique can be described as follows:

Consider two variables, $V$ and $U$, and two errors, $E v$ and $E u$. When $V$ is changed by a small amount $\delta \mathrm{V}$, both errors, $E v$ and $E u$, will consequently change. The error coefficients $(\delta E v / \delta V)$ and $(\delta E u / \delta V)$ are then calculated. $V$ is reset to its initial value and the variable $U$ is changed by $\delta U$. The new error coefficients $(\delta E v / \delta U)$ and $(\delta E u / \delta U)$ are then calculated. Next, the variables $U$ and $V$ are calculated to reduce the errors $E v$ and $E u$ to zero, according to the following equations (GasTurb, 2004): 


$$
\begin{aligned}
& \frac{\delta E u}{\delta U} \Delta U+\frac{\delta E u}{\delta V} \Delta V=-E u \\
& \frac{\delta E v}{\delta U} \Delta U+\frac{\delta E v}{\delta V} \Delta V=-E v
\end{aligned}
$$

\section{Operating line}

This denotes the variation of the performance of the GTE over the entire envelope of operation. This is done by determining the equilibrium running points or operating point at a steady state (steady speed) on the characteristic map of each component. These points are represented for different operating speeds on the compressor and turbine maps forming the operating line (OL). In GasTurb, this line is composed of a series of equidistant points depicted on the component maps. They begin with the calculated single off-design point and spread with equal distances of the high pressure spool speed. The program offers the option of selecting the desired number of points suitable for each application. The operating line is then drawn, connecting these equidistant points. It is worth mentioning that several operating lines can be drawn on the same map depending on the given operating conditions. For example, in a case where the compressor bleed option is incorporated, and all other conditions are invariable, the program will show a new operating line drawn below the normal operating line (the line shown without compressor bleed). These lines indicate how close the GTE running conditions are to the compressor surge line at any given operating speed. Figure 5-1 is a schematic of a 
generic compressor map on which the DP, OD, and OL, amongst other relevant lines, are shown.

\section{Transient performance}

The transient performance or behaviour of the GTE indicates the response of the engine to a change in the output (thrust or power demand) (Cohen et al., 1987). The prediction of this performance is important for control purposes: "A detailed knowledge of the dynamic response at the design stage is becoming increasingly important for the design and development of control systems for advanced gas turbines" (Cohen et al., 1987). The transient behaviour calculations are determined from the off-design performance. These usually include the thermodynamic relations of the steady state GTE model, the polar moment of inertia and the control system. The polar moment of inertia has a direct effect on the work balance between the compressor and the turbine. Because of this, more power is needed from the turbine during transient acceleration than in steady state operation (GasTurb, 2004). The demand for power results in higher fuel flow for a given speed, a shift in the operating line towards the surge line, and in higher operating temperatures (the TIT temperature increases before the compressor reaches its

speed to supply adequate mass flow to the combustor (Cohen et al., 1987). Hence, the control system must be able to keep the fuel flow during transient operation between two limiting schedules: the acceleration schedule (AS) and the deceleration schedule (DS). Figure 5-2 shows a typical transient acceleration schedule on a generic compressor map, and Figure 5-3 is a schematic of a typical compressor map showing all relevant lines and schedules (i.e., SL, OL, AS, and DS). As can be seen in Figure 5-2, when the fuel flow 
increases, the new operating line moves the acceleration schedule closer to the surge line. For control purposes these schedules must be mapped into a gas generator speed $\left(\mathrm{N}_{\mathrm{GG}}\right)$ versus fuel flow ratio graph as shown in Figure 5-4. In the following sections, the control approach using fuzzy logic systems will be covered in detail, including the scheduling of acceleration and deceleration (for transient performance evaluation purposes).

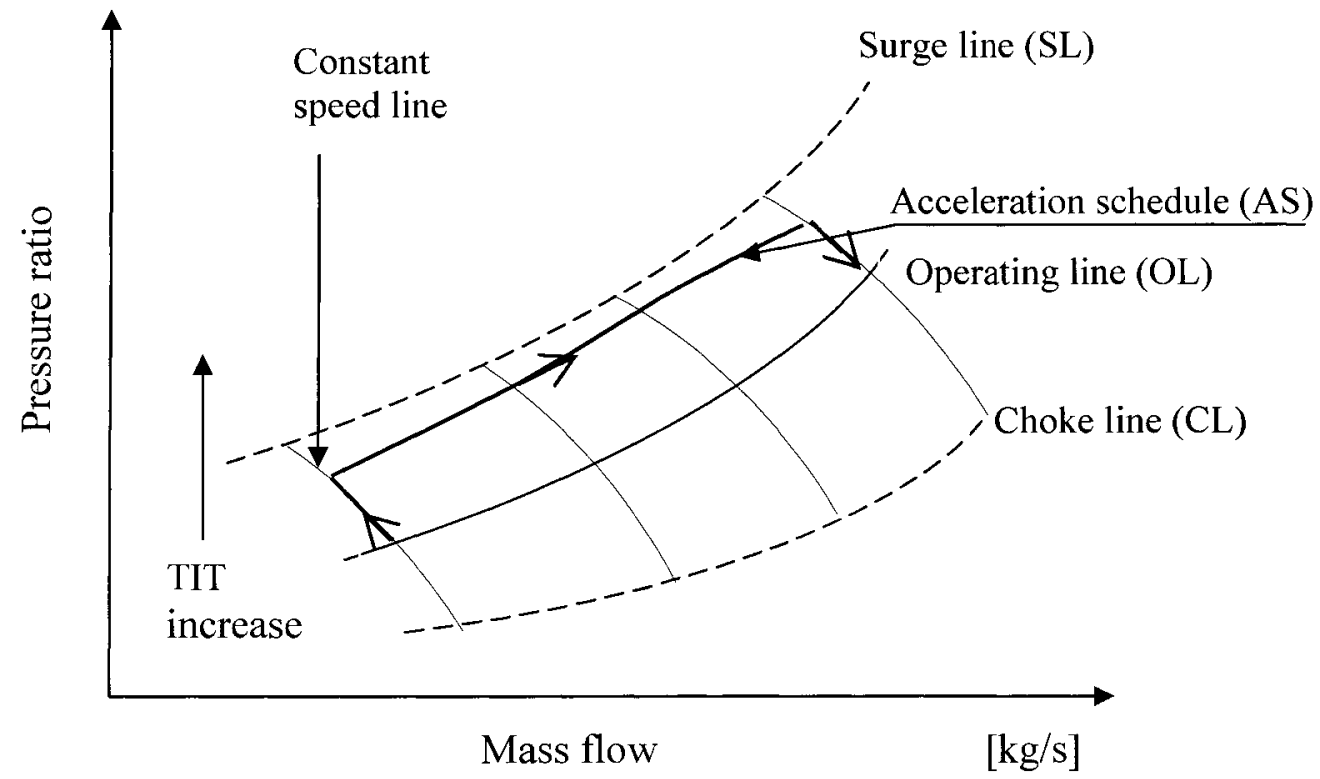

Figure 5-2: Transient acceleration schedule on a compressor map 


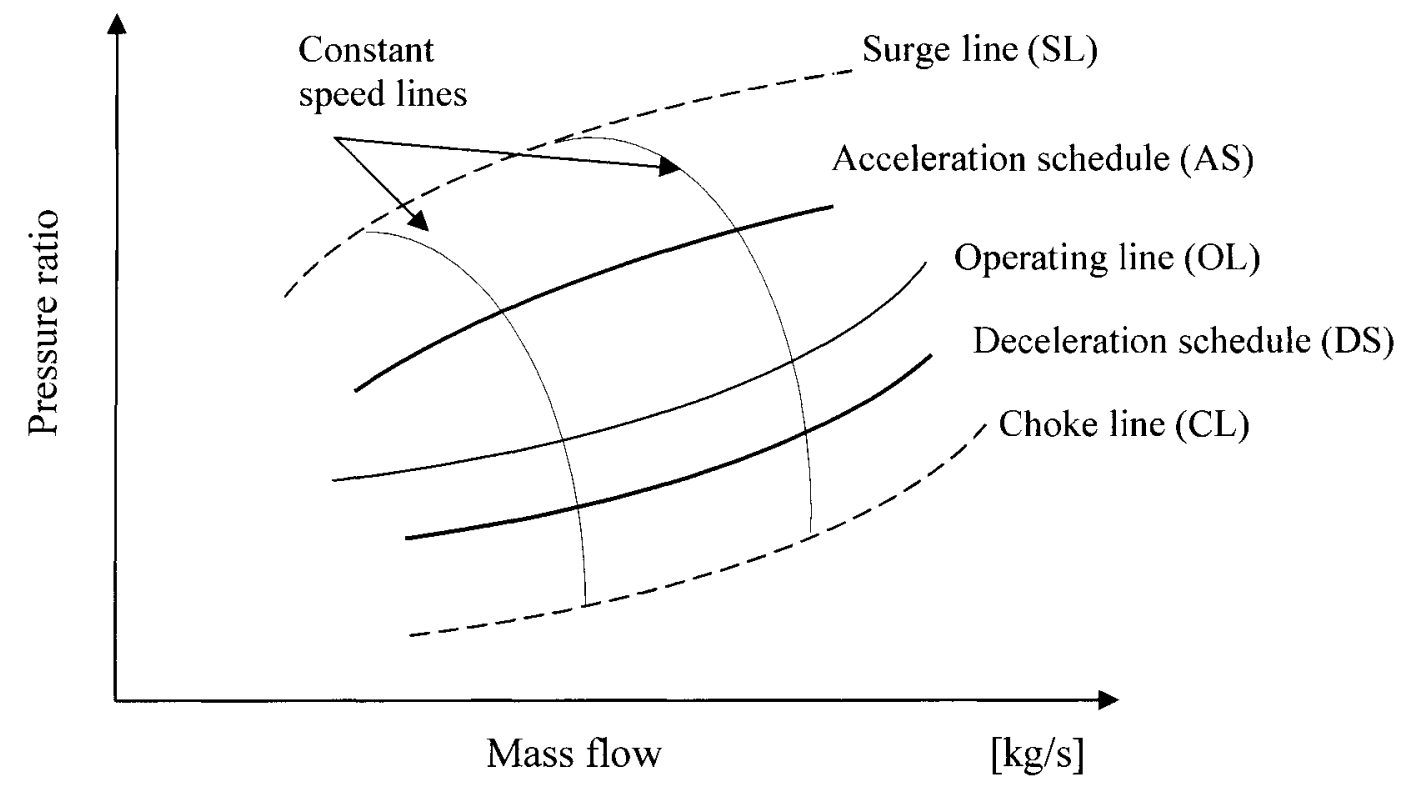

Figure 5-3: Schematic of compressor map with AS and DS shown

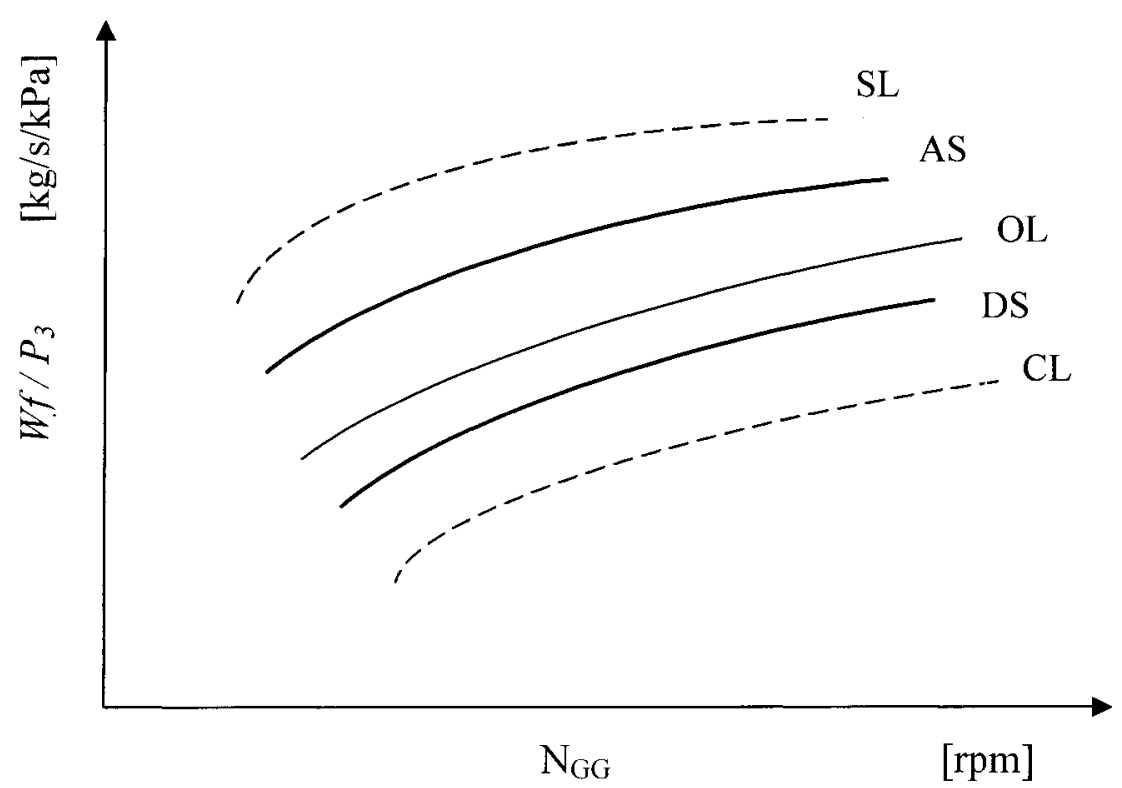

Figure 5-4: Schematic of acceleration and deceleration schedules 


\subsubsection{Problem statement}

As discussed in Chapter 2, the fuel schedules are chosen as limits used in openloop control strategies to avoid surge and the flameout of the gas turbine during transient operation. These schedules are depicted as lines running between the steady-state operating fuel requirements and the surge fuel margin. As can be seen in Figures 5-5 and 5-6, the shapes of these different lines are irregular and therefore can pose computationally challenging problems when implemented the control hardware; this adds further cost and complexity to the controller (GasTOPS, 1990).

In the following sections the selection of a suitable fuel schedule using fuzzy logic will be presented.

\subsubsection{Solution approach}

The gas turbine model and fuel schedules were developed using GasTurb software (GasTurb, 2004), which encompasses a whole series of gas turbine types (turbojet, single and twin spool, turboshaft-turboprop, turbofan, etc.). For each type, a set of design inputs is entailed; the user must select the turbine type and must enter the design parameters as per a predetermined order established by the developer. The parameters in this thesis were sourced and implemented for a twin-shaft turboshaft engine (Table 5-1). These parameters include: the engine mass flow rates, burner pressure ratio, compressor and turbines pressure ratios, inlet temperatures, burner exhaust temperature, compressor and turbines isentropic and polytropic efficiencies, bleed rate, and spool speed. Once all the entries are confirmed, the program generates a design point and an 
off design operating line, which is then illustrated on the turbine and compressor maps generated by the program. Such representations are useful in depicting the relationship between the engine mass flow rate, pressure ratios (in the case of compressor maps), and compressor isentropic efficiency for various operating points at a given operating speed. The values of the formerly listed parameters are central in determining two significant variables used in the controller design, namely, the burner fuel flow $(W f)$ and the compressor discharge pressure $\left(P_{3}\right)$.

Table 5-1: GasTurb parameters for $1 \mathrm{MW}$ twin-shaft turboshaft engine

\begin{tabular}{|l|l|l|l|}
\hline Symbol & Variables Description & Unit & Entry \\
\hline Alt & Altitude & {$[\mathrm{m}]$} & $0.0 \mathrm{E}+0$ \\
\hline Dtamb & Ambient Temperature & {$[\mathrm{K}]$} & $2.88 \mathrm{E}+2$ \\
\hline Humid & Relative Humidity & {$[\%]$} & $0.0 \mathrm{E}+0$ \\
\hline XM & Mach Number & & $0.0 \mathrm{E}+0$ \\
\hline ZW2Rstd & Inlet Corr. Flow W2Rstd & {$[\mathrm{kg} / \mathrm{s}]$} & $5.49 \mathrm{E}+0$ \\
\hline P2q1 & Intake Pressure Ratio & & $9.9 \mathrm{E}-1$ \\
\hline ZP3q2 & Pressure Ratio & & $8.0 \mathrm{E}+0$ \\
\hline T4_D & Burner Exit Temperature & {$[\mathrm{K}]$} & $1.2 \mathrm{E}+3$ \\
\hline e34ds & Burner Design Efficiency & & $9.9 \mathrm{E}-1$ \\
\hline e34C & Burner Partload Constant & & $1.6 \mathrm{E}+0$ \\
\hline FHV & Fuel Heating Value & {$[\mathrm{MJ} / \mathrm{kg}]$} & $4.3 \mathrm{E}+1$ \\
\hline & Fuel Type & & $\mathrm{Generic}$ \\
\hline WHDBLDq2 & Rel. Handling Bleed & & $1.5 \mathrm{E}-2$ \\
\hline hr_hdbldRel. & Enthalpy of Handling Bleed & & $1.0 \mathrm{E}+0$ \\
\hline e_mech_HHP & Spool Mechanical Efficiency & & $9.9 \mathrm{E}-1$ \\
\hline P4q3 & Burner Pressure Ratio & & $9.5 \mathrm{E}-1$ \\
\hline P45q44_r & Turb. Interd. Ref. Press. Ratio & & $1.0 \mathrm{E}+0$ \\
\hline P6q5 & Turbine Exit Duct Press Ratio & & $1.0 \mathrm{E}+0$ \\
\hline P8qamb & Nozzle Pressure Ratio & & $1.0 \mathrm{E}+0$ \\
\hline CFG8 & Nozzle Thrust Coefficient & & $1.0 \mathrm{E}+0$ \\
\hline e_mech_LLP & Spool Mechanical Efficiency & & $9.9 \mathrm{E}-1$ \\
\hline NPTrpm & Nominal PT Spool Speed & {$[\mathrm{RPM}]$} & $2.8 \mathrm{E}+4$ \\
\hline A41r_MEAMe & HPT Flow Area & {$[\%]$} & $0.0 \mathrm{E}+0$ \\
\hline A45r_MEAMe & Power Turb. Flow Area & {$[\%]$} & $0.0 \mathrm{E}+0$ \\
\hline & Ambient Conditions & & Selected \\
\hline
\end{tabular}


Table 5-1: Continued

\begin{tabular}{|l|l|l|l|}
\hline Symbol & Variables Description & Unit & Entry \\
\hline ZT1 & Total Temperature T1 & {$[\mathrm{K}]$} & $2.88 \mathrm{E}+2$ \\
\hline ZP1 & Total Pressure P1 & {$[\mathrm{kPa}]$} & $1.013 \mathrm{E}+2$ \\
\hline ZPamb & Ambient Pressure Pamb & {$[\mathrm{kPa}]$} & $1.013 \mathrm{E}+2$ \\
\hline Zhumid & Relative Humidity & {$[\%]$} & $0.0 \mathrm{E}+0$ \\
\hline & Comp Efficiency & & Selected \\
\hline E23is & Isentr.Compr.Efficiency & & $8.02 \mathrm{E}-1$ \\
\hline & Comp Efficiency & & Selected \\
\hline E23pol & Polytr.Compr.Efficiency & & $8.5 \mathrm{E}-1$ \\
\hline & Comp Efficiency & & Selected \\
\hline NStgHPC & Number of Compr Stages & & $0.0 \mathrm{E}+0$ \\
\hline FLossHPC & Compr Loss Corr Factor & & $0.0 \mathrm{E}+0$ \\
\hline SCDes & Comp Design & & Selected \\
\hline CXN_H & Nominal HPC Spool Speed & & $2.8 \mathrm{E}+4$ \\
\hline SeffHT & HPT Efficiency & & Selected \\
\hline E444is & Isentr.HP Turbine Efficiency & & $8.93 \mathrm{E}-1$ \\
\hline E444pol & Polytr.HP Turbine Efficiency & & $8.8 \mathrm{E}-1$ \\
\hline SEffPT & PT Efficiency & & Selected \\
\hline E455is & Isentr.Power Turbine Eff. & & $8.9 \mathrm{E}-1$ \\
\hline E455pol & Polytr.Power Turbine Eff. & & $8.8 \mathrm{E}-1$ \\
\hline STestAn & Test Analysis & & Selected \\
\hline P3 m & Measured P3 & {$[\mathrm{kPa}]$} & $8.026 \mathrm{E}+2$ \\
\hline T3_m & Measured T3 & {$[\mathrm{K}]$} & $5.743 \mathrm{E}+2$ \\
\hline WF_m & Measured Fuel Flow & {$[\mathrm{kg} / \mathrm{s}]$} & $8.91 \mathrm{E}-2$ \\
\hline P44_m & Measured P44 & {$[\mathrm{kPa}]$} & $2.558 \mathrm{E}+2$ \\
\hline PWSD_m & Measured Shaft Power & {$[\mathrm{kW}]$} & $1.012 \mathrm{E}+3$ \\
\hline
\end{tabular}

Figure 5-5 illustrates the generic compressor map generated by GasTurb software. This graph will be used as a template for the proposed design. The goal of choosing an acceleration schedule is to limit the fuel to the engine at each operating point to a safe value. One way to achieve a safe margin of operation is to map the limiting lines shown on the compressor map onto a gas generator speed versus fuel flow ratio graph as per Figure 5-6. To do so, the GasTurb software option to transfer the data into a workable Excel spreadsheet was used (Appendix B includes the generated values). The relation 
between the speed and $\mathrm{WflP}_{3}$ is then drawn in the form of a graph using the chart option available in Excel (Figure 5-6). The next step is to draw an acceleration schedule between the surge and operating lines. In the case presented here, the acceleration schedule was selected midway between the surge line and the operating line. This selection is reasonable since it secures a compromise between a fast acceleration and safety.

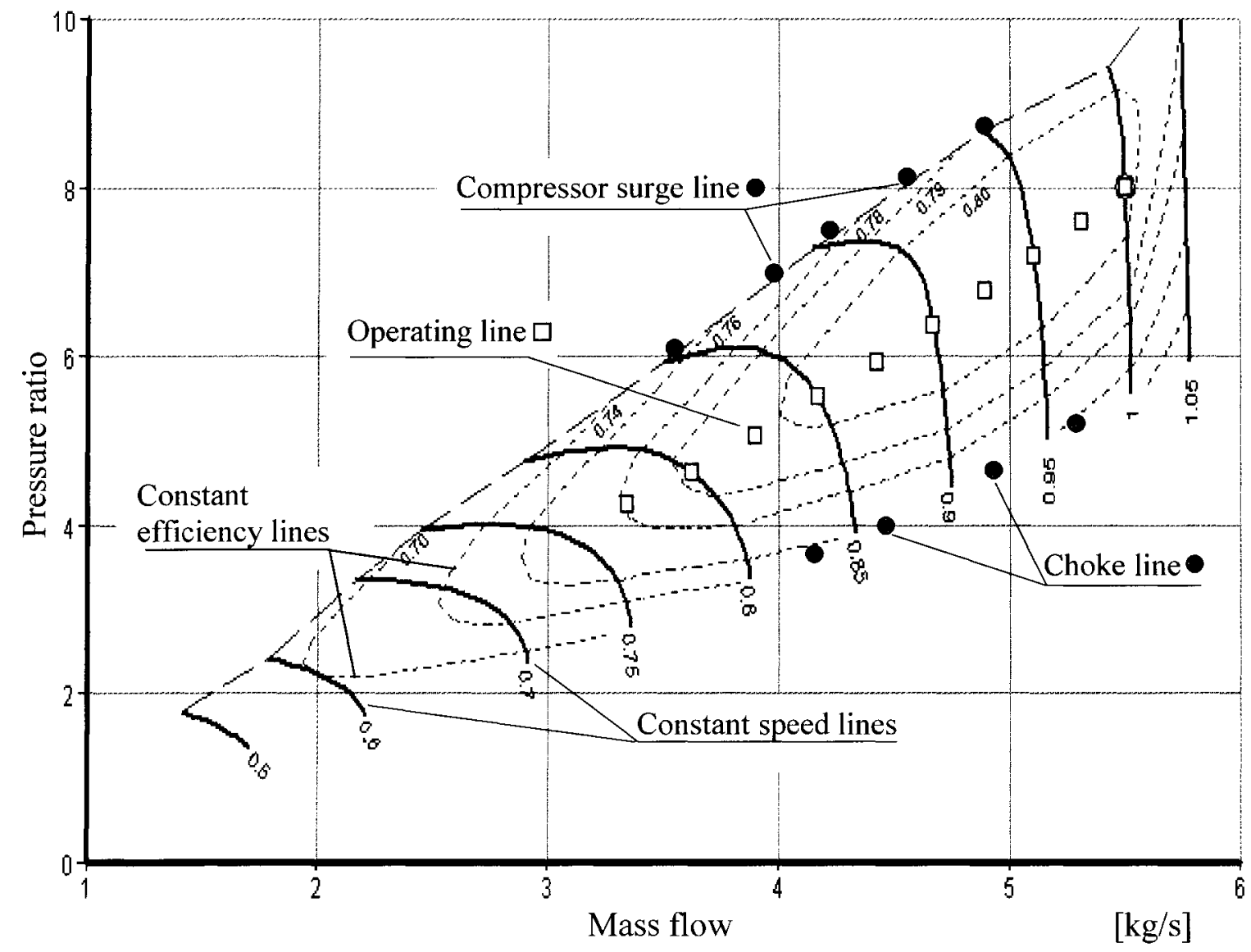

Figure 5-5: Compressor map with surge, operating, and deceleration schedule lines shown (generated from GasTurb) 


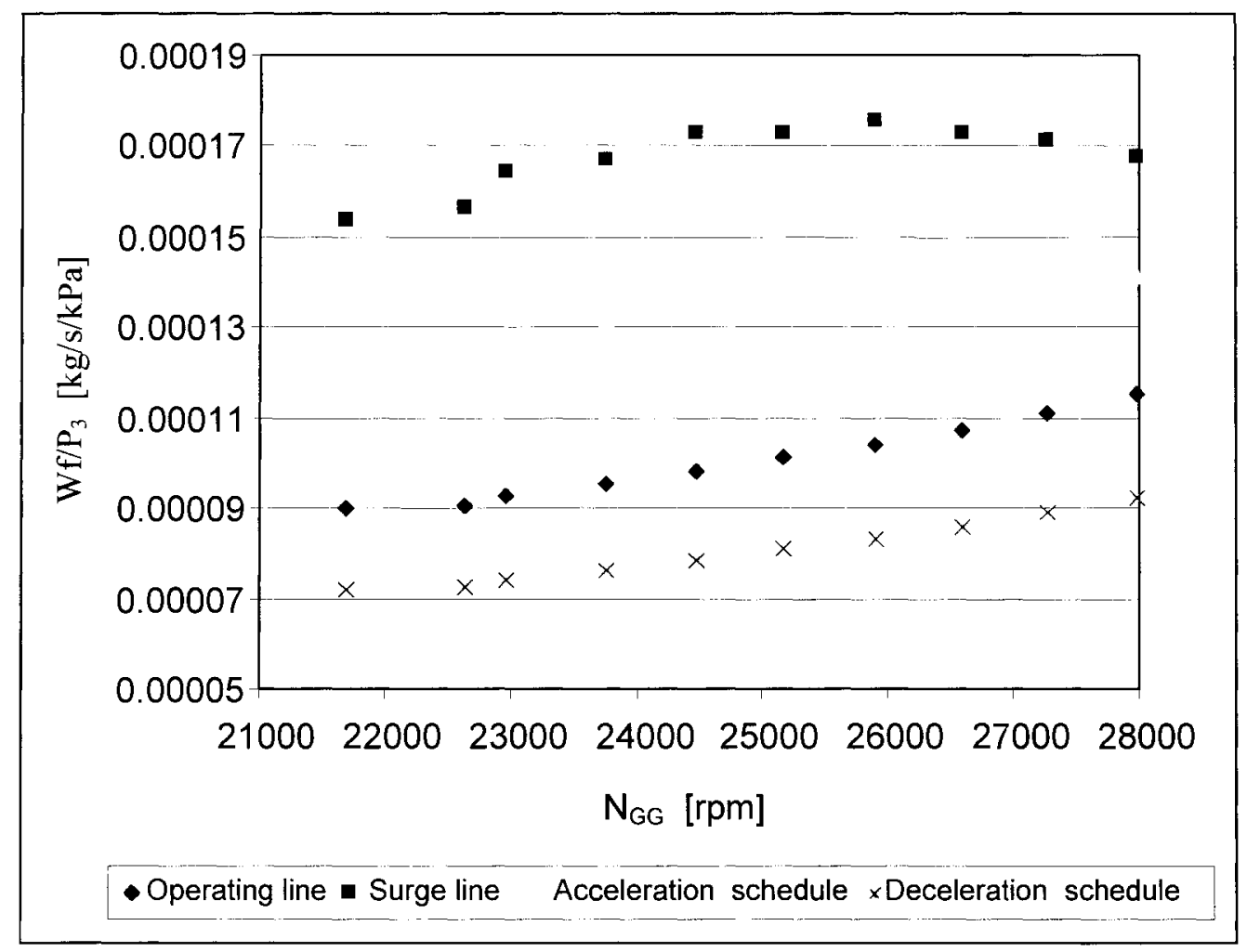

Figure 5-6: Fuel schedule on a fuel flow ratio versus gas generator speed $\left(\mathrm{N}_{\mathrm{GG}}\right)$ graph

A similar procedure is applied to the selection of the deceleration schedule. However, in this case the surge line is exchanged with the choke line. The choice of the deceleration schedule is somewhat arbitrary, but constrained by flame stability and choke point. In this thesis, a deceleration schedule at $20 \%$ below the operating line was chosen (the $20 \%$ selection will be revised if necessary when the combustor is tested and the flameout line [flame stability] and chock line are defined). 
Now that the schedules have been marked analytically, a fuzzy logic approach is developed to map these fuel schedules.

\subsection{Fuzzy logic control design}

Fuzzy logic applications in control, in a broader perspective, can be looked at as deriving the output control action from the input in the form of degrees of membership (negative, zero, and positive). These membership functions can be devised from either expert knowledge, or from experimental data collected from measurements and coupled with expert information.

In this chapter, the expert knowledge approach is considered, since for this controller design there is interest in producing a relation between input and output in the form of a curve. In other words, the task is to define the rule-base and membership functions of the FLC to merely curve-fit a set of data related to the controller design. In doing so, a fuel schedule that can be easily modified to suit any further design requirements will be generated. The input output variables are the gas turbine compressor rotational speed, termed the gas generator speed $\left(\mathrm{N}_{\mathrm{GG}}\right)$ and the fuel flow ratio unit, abbreviated by $W f / P_{3}$. The simulation model and the Matlab codes are included in Appendix C. The following steps are implemented for the acceleration schedule.

\subsubsection{Define inputs and outputs for the control scheme}

The linguistic fuzzy model (the Mamdani model) is employed. The advantage of this fuzzy model is that both the antecedent and the consequent are fuzzy propositions. For the proposed design, the if-then rule is expressed in the general form: 
if $\mathbf{N}_{\mathbf{G G}}$ is $A_{i}$ then $\mathbf{W} \mathbf{f} / \mathbf{P}_{\mathbf{3}}$ is $B_{i}, \quad i=1,2, \ldots, K$,

where $\mathbf{N}_{\mathbf{G G}}$ is the antecedent variable representing the input to the fuzzy controller, and $\mathbf{W f} / \mathbf{P}_{\mathbf{3}}$ is the consequent variable depicting the output of the fuzzy system. $K$ is the number of rules in the rule-base. Both antecedents and consequents are fuzzy linguistic variables having fuzzy sets as their values. In this case, $A_{i}$ and $B_{i}$ are considered to be two fuzzy sets defined as linguistic terms by the membership functions $\mu_{A i}\left(\mathrm{~N}_{\mathrm{GG}}\right)$ and $\mu_{B i}\left(\mathrm{Wf} / \mathrm{P}_{3}\right)$, respectively, where:

$$
\begin{aligned}
& \mathrm{N}_{\mathrm{GG}} \rightarrow[0,1], \\
& \mathrm{Wf} / \mathrm{P}_{3} \rightarrow[0,1]
\end{aligned}
$$

\subsubsection{Fuzzy system process}

To explain how the fuzzy system process works, a brief description follows.

After the input output linguistic variables and rules are specified, the membership functions are defined in a database. The combination of the database and the rule-base creates the knowledge base in which all the information related to the fuzzy system is stored. The input output mapping is then generated in the fuzzy inference engine. This mechanism allows an output value to be created from an input value in conjunction with the knowledge amassed in the knowledge base. For example:

$$
\text { if } \mathbf{N}_{\mathbf{G G}} \text { is }<\text { low }>\text { then } \mathbf{W} \mathbf{f} / \mathbf{P}_{\mathbf{3}} \text { is }<\text { small }>
$$

where the corresponding value of $\mathbf{N}_{\mathbf{G G}}$ in the $<$ low $>$ range is between 21000 and 23000 $\mathrm{rpm}$ and the output value of $\mathbf{W} \mathbf{f} / \mathbf{P}_{\mathbf{3}}$ in the $<$ small $>$ interval is between $5 \mathrm{E}-05$ to $7 \mathrm{E}-05$ $\mathrm{kg} / \mathrm{s} / \mathrm{kPa}$, as shown in Figure 5-6. The values of $\mathbf{N}_{\mathbf{G G}}$ and $\mathbf{W} \mathbf{f} / \mathbf{P}_{\mathbf{3}}$ come from the GasTurb 
simulation. Depending on the membership functions, rules and rule firing strength, the exact output value for a given input can be determined.

\subsubsection{Linguistic terms}

Klir and Yuan 1995, gave the following definition of the linguistic terms.

$$
\mathrm{L}=(\chi, A, X, g, \tilde{A})
$$

Where:

$\chi$ is the given variable,

$A=\left\{\mathrm{A}_{1}, \mathrm{~A}_{2}, \ldots, \mathrm{A}_{\mathrm{N}}\right\}$ is the set of linguistic terms of $\chi$,

$X$ is the universe of discourse of the given variable,

$g$ defines a syntactic rule,

$\tilde{A}$ is a fuzzy set in $X$ that assigns values to each linguistic term.

Figure 5-7 illustrates the linguistic variable $\mathbf{N}_{\mathbf{G G}}$ with five linguistic terms $<$ negative big (NB) $>,<$ negative medium $(\mathrm{NM})>,<$ zero $(\mathrm{ZR})>,<$ positive small $(\mathrm{PS})>$ and $<$ positive $(\mathrm{P})>$. For every linguistic term the membership functions overlap. In other words, each element is allocated to one or more fuzzy sets with a nonzero membership degree. This is referred to as coverage (Pedrycz, 1995) on a fuzzy partition map. 

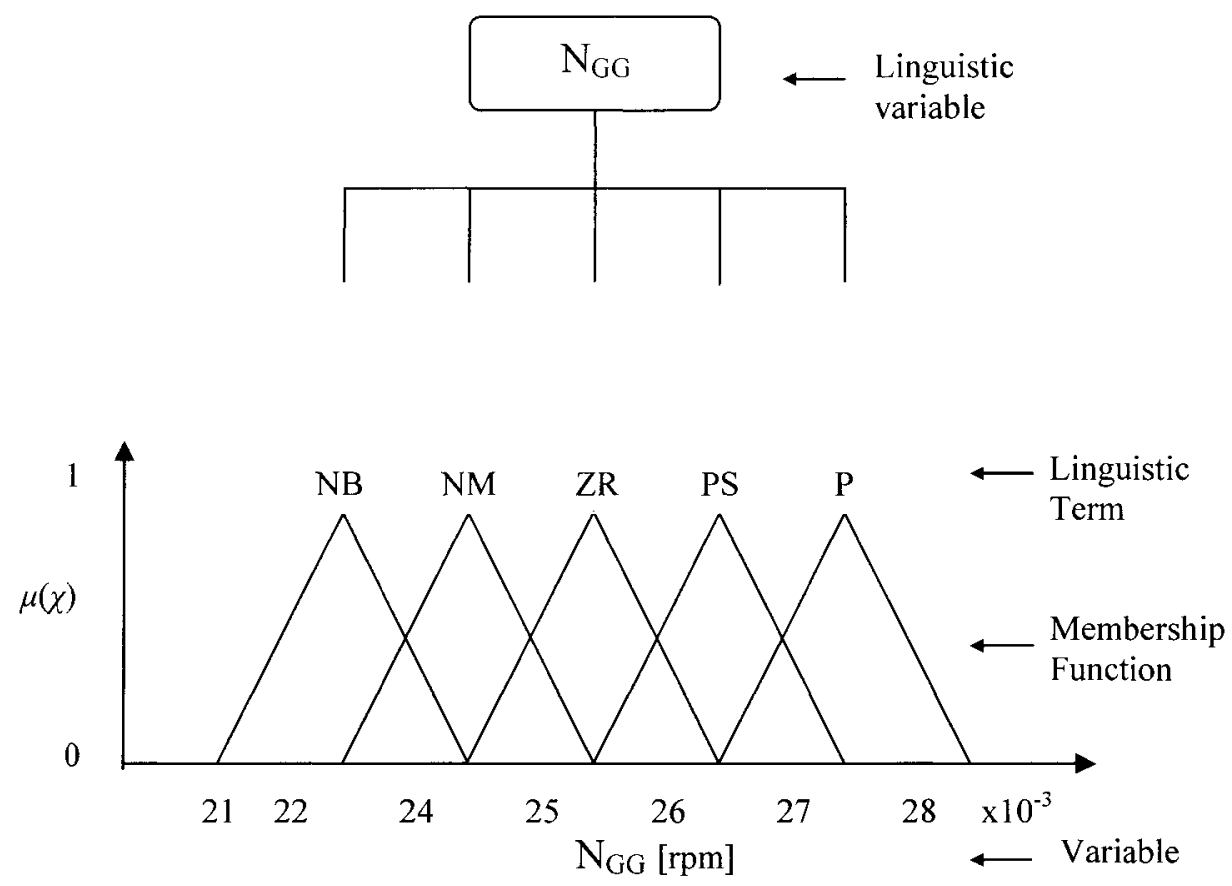

Figure 5-7: Linguistic variable $\mathrm{N}_{\mathrm{GG}}$ with five linguistic terms

\subsubsection{Antecedent-consequent propositions}

In this section two linguistic variables are used, $\mathbf{N}_{\mathbf{G G}}$ and $\mathbf{W f} / \mathbf{P}_{\mathbf{3}}$. One method of representing the antecedent-consequent relation (or rule-base) is through a fuzzy matrix. Table 5-2 shows a one-dimensional representation of the fuzzy sets implemented for the acceleration schedule.

Table 5-2: Rule-base for fuel flow membership functions; acceleration schedule (Mamdani fuzzy inference system).

\begin{tabular}{|l|c|c|c|c|c|c|c|c|}
\hline $\mathrm{N}_{\mathrm{GG}}$ & $\mathrm{NS}$ & $\mathrm{N}$ & $\mathrm{NM}$ & Zero & PS & PM & $\mathrm{P}$ & $\mathrm{PB}$ \\
\hline $\mathrm{Wf} / \mathrm{P}_{3}$ & $\mathrm{LN}$ & $\mathrm{N}$ & $\mathrm{NS}$ & $\mathrm{NS}$ & Zero & $\mathrm{P}$ & $\mathrm{PS}$ & $\mathrm{PB}$ \\
\hline
\end{tabular}




\subsubsection{Fuzzy membership functions}

The fuzzy membership functions characterizing the consequent rules are shown in Table 5-3. The overlapping in the membership functions are intended to guarantee a smooth mapping and transition between the discrete and desired control actions. The values are drawn from the GasTurb simulation results.

Table 5-3: Fuzzy output control value (Acceleration schedule)

\begin{tabular}{|c|c|c|}
\hline Shape & $\begin{array}{l}\text { Fuzzy logic values interval } \\
\qquad \mathrm{Wf} / \mathrm{P}_{3}[\mathrm{~kg} / \mathrm{s} / \mathrm{kPa}]\end{array}$ & $\begin{array}{l}\text { Linguistic term } \\
\text { description }\end{array}$ \\
\hline Triangular & 11.5 to 12.5 e-5 & LN (low negative) \\
\hline Triangular & 12.3 to $12.85 \mathrm{e}-5$ & $\mathrm{~N}$ (negative) \\
\hline Triangular & 12.5 to 13.25 e-5 & NS (negative small) \\
\hline Triangular & 12.85 to $13.5 \mathrm{e}-5$ & Zero \\
\hline Triangular & 13.25 to $13.85 \mathrm{e}-5$ & $\mathrm{P}$ (positive) \\
\hline Triangular & 13.5 to 14 & PS (positive small) \\
\hline Triangular & 13.85 to $14.5 \mathrm{e}-5$ & PB (positive big) \\
\hline
\end{tabular}

Figures 5-8 and 5-9 show the membership functions for input speed vector $\left(\mathrm{N}_{\mathrm{GG}}\right)$ and the membership functions for the output fuel ratio $\left(\mathrm{Wf} / \mathrm{P}_{3}\right)$ for the acceleration schedule, respectively. The rule-base is as per Table 5-2. 


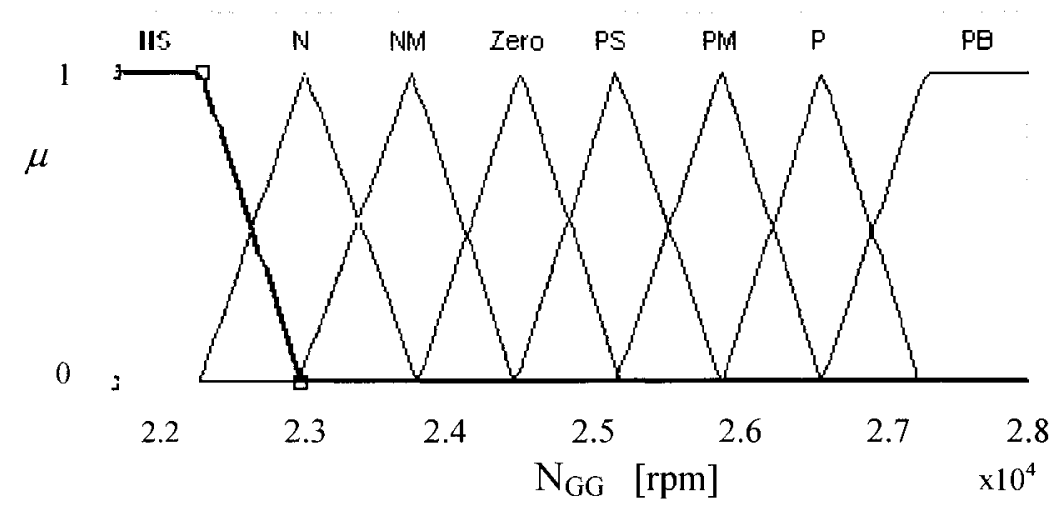

Figure 5-8: Membership functions for $\mathrm{N}_{\mathrm{GG}}$ input vector

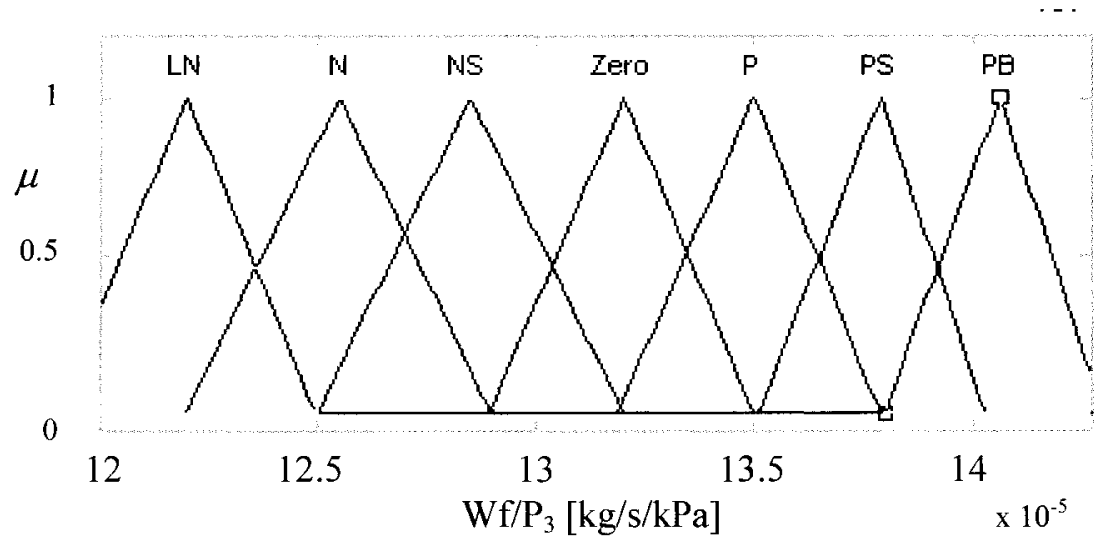

Figure 5-9: Membership functions for fuel ratio output vector

\subsubsection{Mamdani system application to FLC acceleration schedule}

The following represents the Mamdani max-min inference mechanism applied to the fuel schedule problem (with $m$ number of propositions):

- Degree of firing of the antecedent, for each rule $i$, is determined by:

$$
\lambda_{i}=\min \mu_{A i j}\left(\chi_{j}\right) \quad j=1, \ldots, m
$$

- The minimum $T$-norm is used to compute the consequent fuzzy set $\check{Z}_{i}$ for each 
rule, as follows:

$$
\mu_{Z i}(z)=\min \left(\lambda_{i,}, \mu_{Z i}(z)\right), \quad \text { for } z \in Z
$$

- The aggregation is accomplished by using the union (maximum) operator to calculate the output set from all consequents $\check{Z}_{i}$, as defined in the following equation:

$$
\underset{i \in[1, \mathrm{~m}]}{\mu \grave{Z}(z)=\max } \mu_{\check{Z} i}(z), \text { for } z \in Z
$$

Defuzzification is based on the centroid-of-area and transforms the fuzzy set to a single numerical value via equation (3-2-17). The last figure in this section, Figure 5-10, illustrates the results of the fuzzy acceleration schedule versus the analytical schedule. As can be seen, the FLC curve (yellow) matches the analytical curve to some extent. The discrepancies can be abridged by fine-tuning the fuzzy membership functions.

Now that the acceleration schedule has been devised, the fuzzy system application will be extended to cover the design of the deceleration schedule. The following section will detail the deceleration schedule realization with a Sugeno fuzzy inference system. 


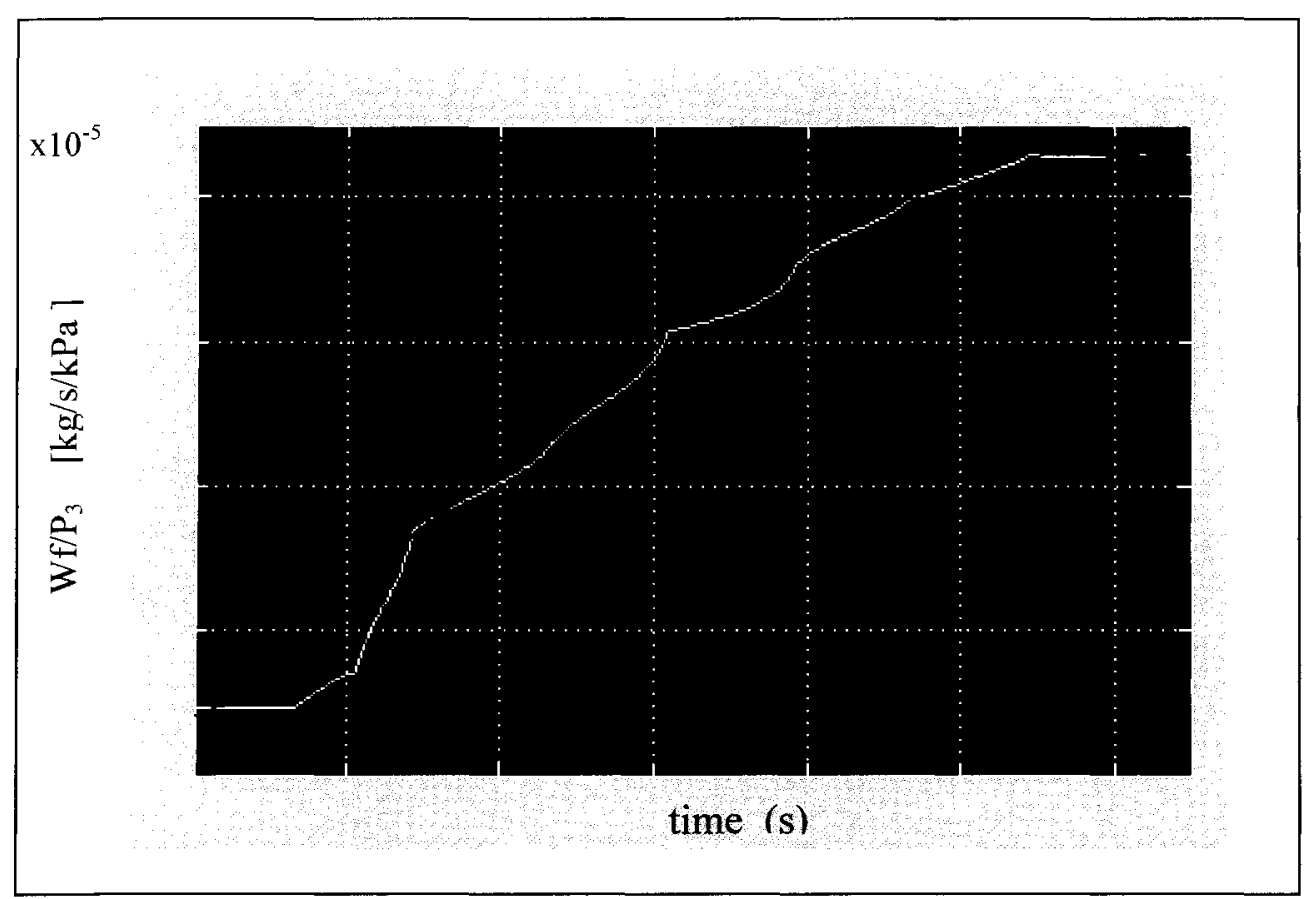

Figure 5-10: Fuzzy acceleration response (yellow) versus GasTurb response (red)

\subsubsection{Sugeno fuzzy inference applied to the deceleration schedule}

For the design of the deceleration fuel schedule, a Sugeno fuzzy system with constant consequent membership functions was employed. Table 5-4 lists the rule-base for the deceleration fuel schedule, and Table 5-5 itemizes the fuzzy output values $\left(\mathrm{Wf} / \mathrm{P}_{3}\right)$

Table 5-4: Rule-base for deceleration fuel schedule (Sugeno fuzzy system)

\begin{tabular}{|l|c|c|c|c|c|c|c|}
\hline$N_{\text {GG }}$ & NS & N & NB & Zero & PS & P & PB \\
\hline $\mathrm{Wf} \mathrm{P}_{3}$ & $\mathrm{NS}$ & $\mathrm{N}$ & $\mathrm{NB}$ & Zero & PS & $\mathrm{P}$ & $\mathrm{PB}$ \\
\hline
\end{tabular}


Figures 5-11 and 5-12 represent the membership functions of the input $\left(\mathrm{N}_{\mathrm{GG}}\right)$ and output (fuel ratio) vectors for the Sugeno fuzzy system, respectively. Figure 5-13 illustrates the deceleration schedule predicted by the fuzzy reasoning (yellow) as compared to the analytical fuel schedule (purple). A closely accurate fit was achieved with Sugeno inference engine.

Table 5-5: Fuzzy output control value (Deceleration schedule)

\begin{tabular}{|l|cc|l|}
\hline Shape & $\begin{array}{c}\text { Fuzzy logic values interval } \\
\text { Wf } / \mathrm{P}_{3}[\mathrm{~kg} / \mathrm{s} / \mathrm{kPa}]\end{array}$ & Linguistic term description \\
\hline Constant & $7.259 \quad \mathrm{e}-5$ & NB (negative big) \\
\hline Constant & $7.408 \quad \mathrm{e}-5$ & N (negative) \\
\hline Constant & $7.612 \quad \mathrm{e}-5$ & NS (negative small) \\
\hline Constant & 7.825 & $\mathrm{e}-5$ & Zero \\
\hline Constant & 8.255 & $\mathrm{e}-5$ & PS (positive small) \\
\hline Constant & 8.7 & $\mathrm{e}-5$ & P (positive) \\
\hline Constant & 9.235 & $\mathrm{e}-5$ & PB (positive big) \\
\hline
\end{tabular}




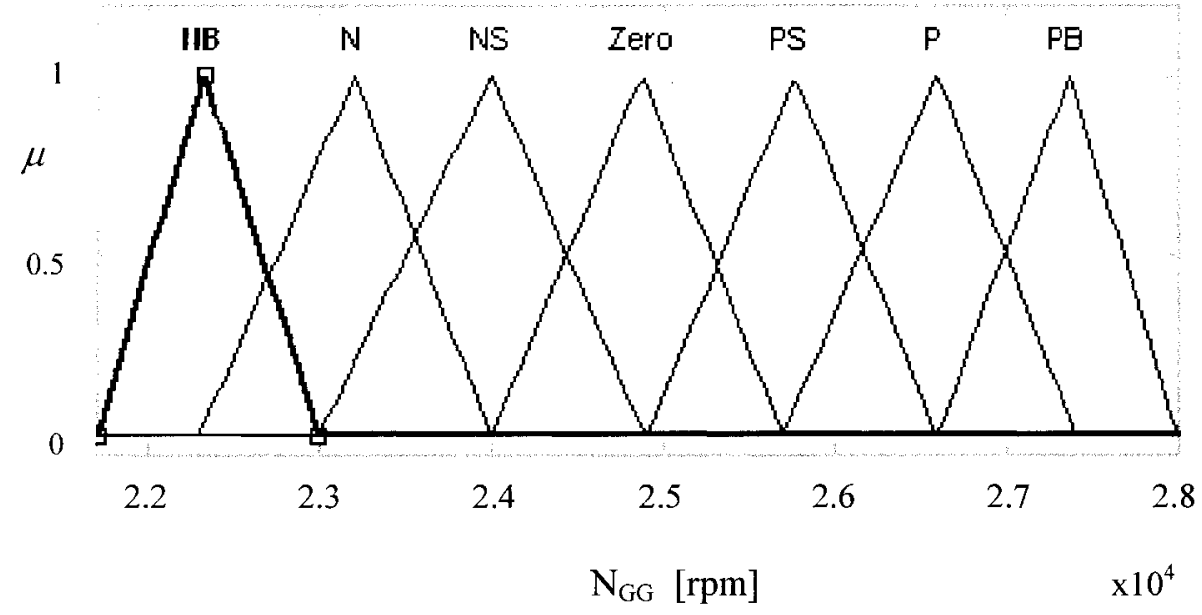

Figure 5-11: Input vector $\mathrm{N}_{\mathrm{GG}}$ membership functions; deceleration (Sugeno)

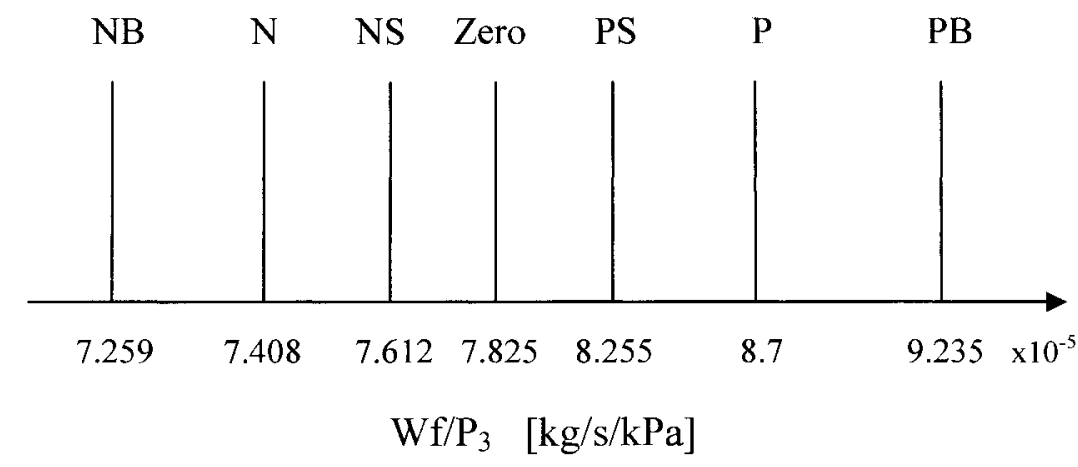

Figure 5-12: Output membership functions (fuel ratio unit); deceleration (Sugeno) 


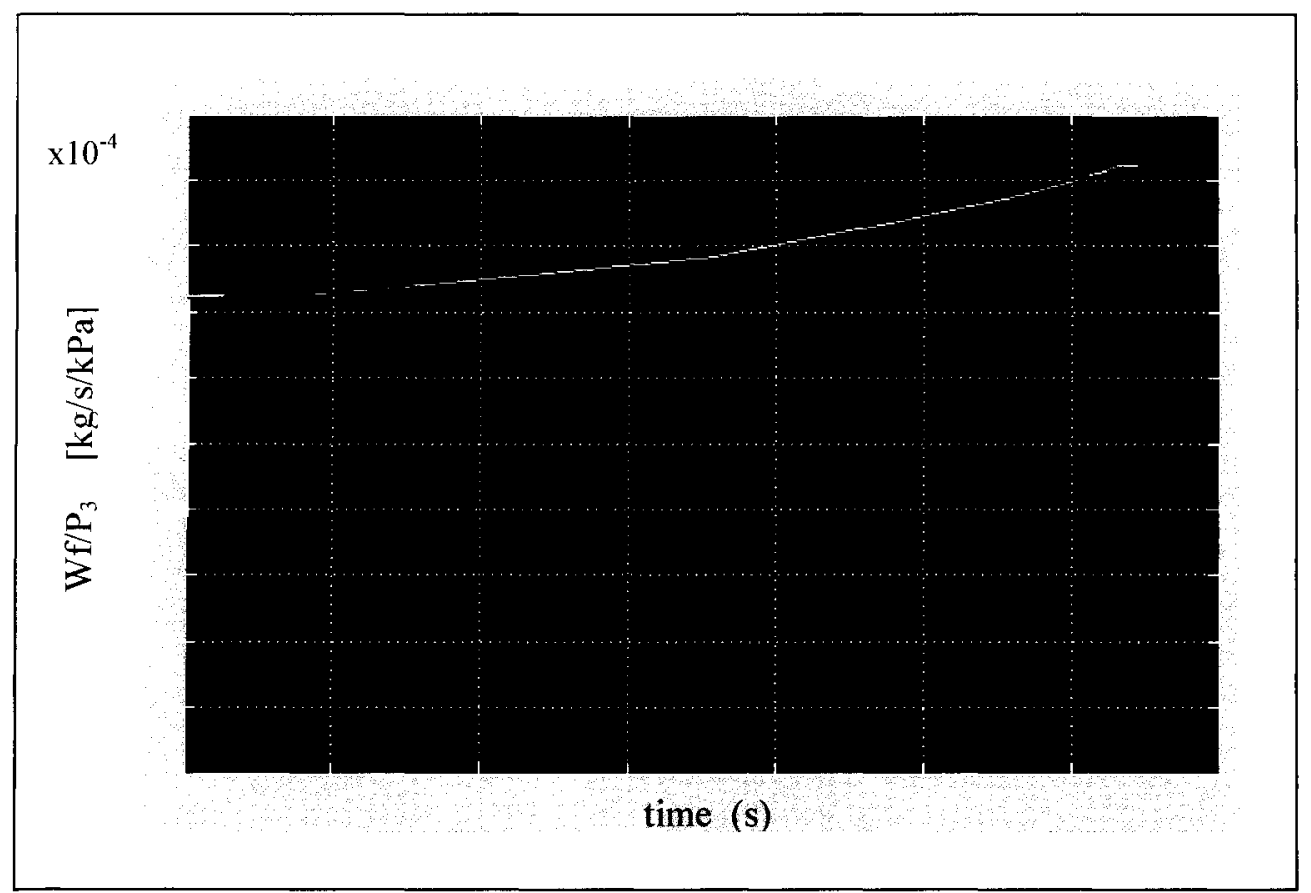

Figure 5-13: Fuzzy deceleration response (yellow) versus GasTurb response (red)

\subsection{Application of fuzzy control to the thermodynamic model}

It is feasible to apply some of the knowledge presented in the previous sections to design a controller for a general thermodynamic gas turbine engine model. In this example a model is derived from the "Zero-Emission GT" simulation developed at Carleton University and the controller is discussed in the subsequent section. The reason behind this selection is the model's availability.

The gas turbine engine model is included in Appendix D. It consists of a gas turbine engine rated at $60 \mathrm{~kW}$. The GTE rated speed is $110000 \mathrm{rpm}$ and it is controlled by a fixed gains PID. The purpose of this section is to demonstrate the ability of the fuzzy logic control approach to control a GTE in a predefined range of operation. The controller design requirement is to maintain the GTE speed at the rated speed $(110000$ 
$\mathrm{rpm}$ ) by varying the fuel flow input to the gas turbine engine at any given power. The design steps are as summarized in the next subsection.

\subsubsection{Fuzzy control strategy}

For this application, the Sugeno fuzzy inference engine is selected. The input variables to the fuzzy logic controller are the gas generator speed error (Errorspeed) (i.e. the difference between the rated GG speed of $110000 \mathrm{rpm}$ and the actual GG speed), and the rate of change of that error (Errordot). The output control action is the change of fuel flow (Wf). Figures 5-14, 5-15, and 5-16 show the inputs and output membership functions. The values are from the "Zero-Emission GT" simulation program. Depending on the rate of change of the error and its magnitude, the fuzzy logic controller commands a fuel flow change. The objective of the controller is to keep the operating GG speed at its rated value $(110000 \mathrm{rpm})$ under varying power conditions. In the case presented subsequently the response of the GTE to change in power (reduction in power or load) is shown.

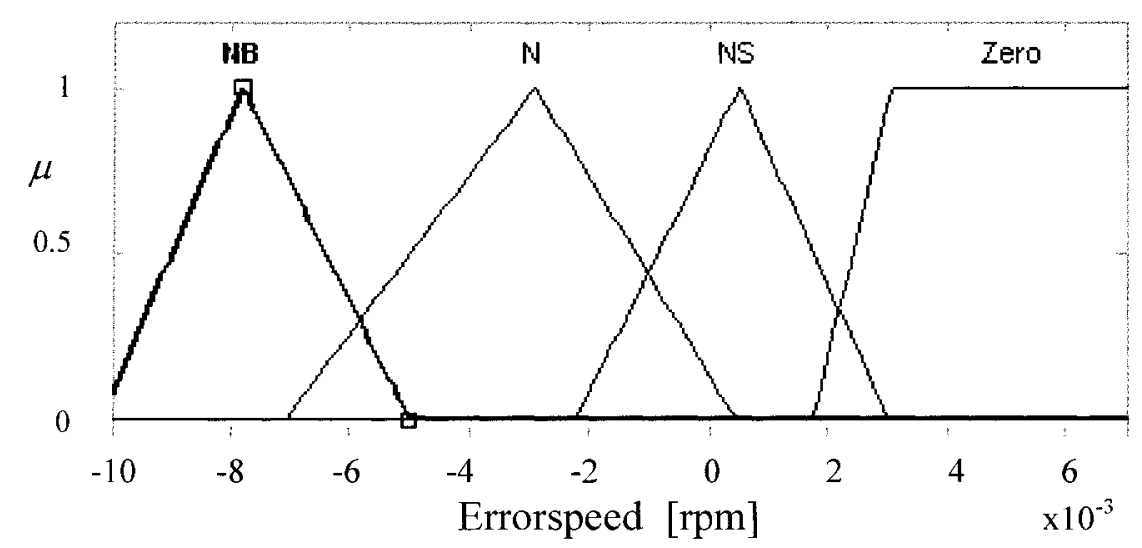

Figure 5-14: Errorspeed input membership function 


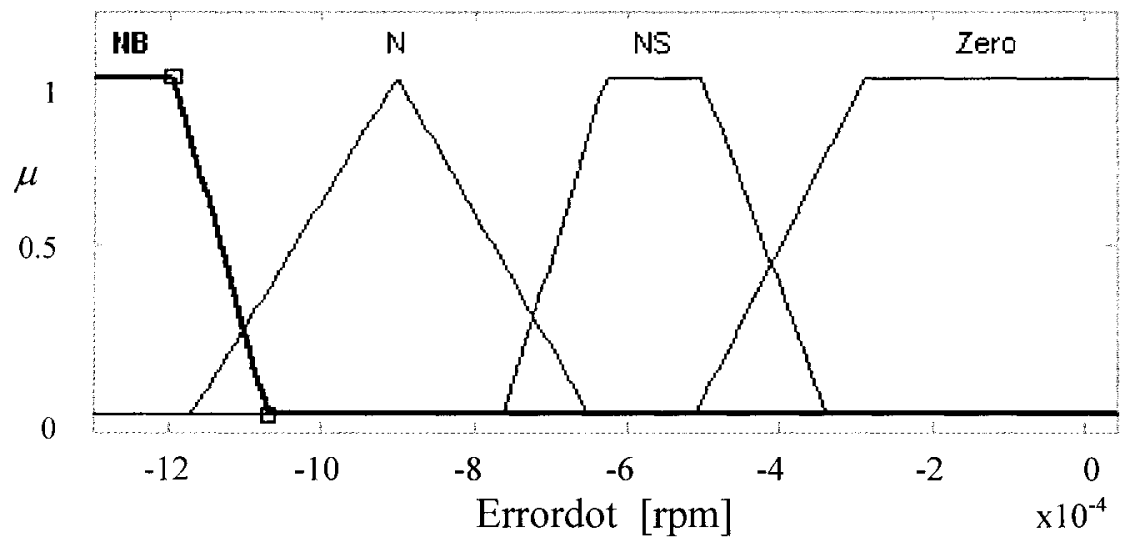

Figure 5-15: Errordot input membership function

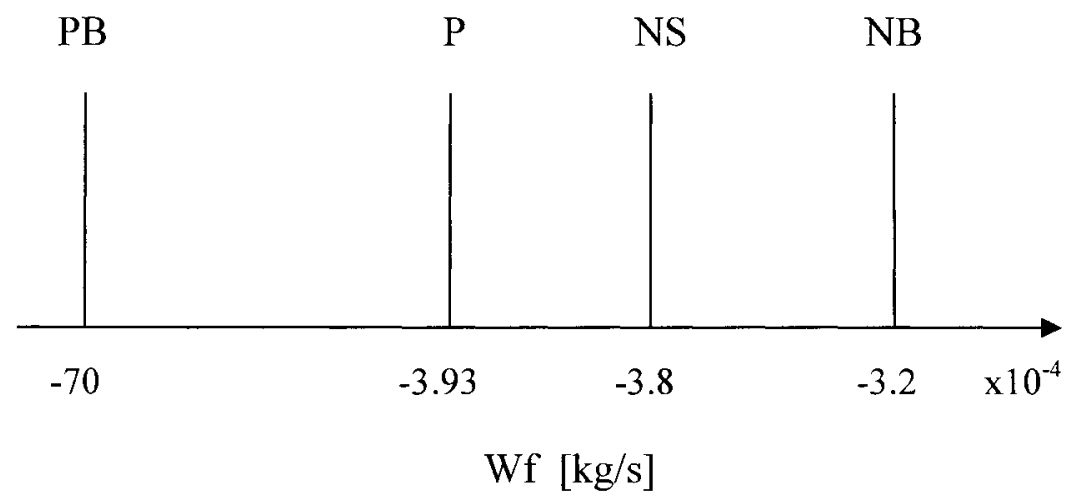

Figure 5-16: Fuel flow output membership functions (Zero-Emission GT)

Table 5-6 lists the fuel flow output control values, and Table 5-7 includes the rules devised for the control strategy. There are 16 rules relating two inputs with 4 membership functions each. 
Table 5-6: Fuel flow output control values (Zero-Emission GT)

\begin{tabular}{|l|cc|l|}
\hline Shape & \multicolumn{2}{|c|}{$\begin{array}{r}\text { Fuzzy logic values interval } \\
\text { Wf }[\mathrm{kg} / \mathrm{s}]\end{array}$} & Linguistic term description \\
\hline Constant & -3.2 & $\mathrm{e}-4$ & NB (negative big) \\
\hline Constant & -3.8 & $\mathrm{e}-4$ & NS (negative small) \\
\hline Constant & -3.93 & $\mathrm{e}-4$ & $\mathrm{P}$ (positive) \\
\hline Constant & -70 & $\mathrm{e}-4$ & PB (positive big) \\
\hline
\end{tabular}

In Table 5-7, the first row and first column denote the antecedent in a rule. The consequent are shown inside the bordered square. A rule, for example, can be read as follows:

if $<$ Errorspeed is $\mathbf{N}$ and Errordot is $\mathbf{N S}>$ then $<\mathbf{W f}$ is $\mathbf{P B}>$.

Table 5-7: Rule-base for fuel flow change (Sugeno)

\begin{tabular}{|l|c|c|c|c|}
\hline $\begin{array}{c}\text { Errorspeed } \\
\text { LErrordot }\end{array}$ & NB & $\mathrm{N}$ & NS & Zero \\
\hline $\mathrm{NB}$ & $\mathrm{NB}$ & $\mathrm{PB}$ & $\mathrm{P}$ & $\mathrm{NB}$ \\
\hline $\mathrm{N}$ & $\mathrm{P}$ & $\mathrm{P}$ & $\mathrm{NB}$ & $\mathrm{P}$ \\
\hline $\mathrm{NS}$ & $\mathrm{NS}$ & $\mathrm{PB}$ & $\mathrm{PB}$ & $\mathrm{NS}$ \\
\hline Zero & $\mathrm{PB}$ & $\mathrm{NS}$ & $\mathrm{P}$ & $\mathrm{NB}$ \\
\hline
\end{tabular}




\subsubsection{Simulation results}

The performance of the fuzzy controller and of the linear PID controller are evaluated at a determined GTE power rating operating condition and the responses are determined. The PID controller gains are determined using Ziegler-Nichols correlation method. This method requires that the proportional gain be determined first by implementing a proportional control loop and varying the proportional gain until a maintainable oscillating response is reached. The integral and the derivative gains are then calculated. The fine tuning of the gains is then performed to guarantee the desired system response. The response of the GTE was examined with different PID gains and the selected controller gains are as follows:

- Proportional gain: 2.7e-07

- Integral gain: 1.35e-08

- Derivative gain: $4.32 \mathrm{e}-07$

The program (see Appendix D) generated the output shown in Figures 5-17 (PID response) and 5-18 (FLC response). The curves in these two figures show the response of the GTE when a load of $50 \mathrm{~kW}$ is disengaged. As the load is removed the GTE accelerates and the GG speed increases until the control action of the controller reduces it back to its rated value (110000 rpm). By simply observing the two figures, one can conclude that the implemented fuzzy logic controller (Figure 5-18(a)) has a settling time of 80 seconds. However, the settling time and the overall response of the controlled system can be changed by simply modifying the FLC membership functions as shown in Figure 5-18(b). The settling time in Figure 5-18(b) is 70 seconds and the modified 
membership functions are included in Appendix D (only the input membership functions were tuned). The FLC range can be extended to cover the entire operating envelop of the GTE. In practical applications, PID with variable gains (gain schedule) is used to control nonlinear dynamic systems and particularly the GTE. In general, the control of the gas turbine engine can be improved by changing the fuel setting input in relation to the shaft speed. Accurate models of the GTE are required before a control scheme is developed. Thus, an artificial neural network, as will be seen in the following section, is a promising tool in duplicating the relation between gas generator speed and fuel flow in gas turbine engines. It can therefore be regarded as a useful modelling technique used to generate gas turbine models from input output data sets.

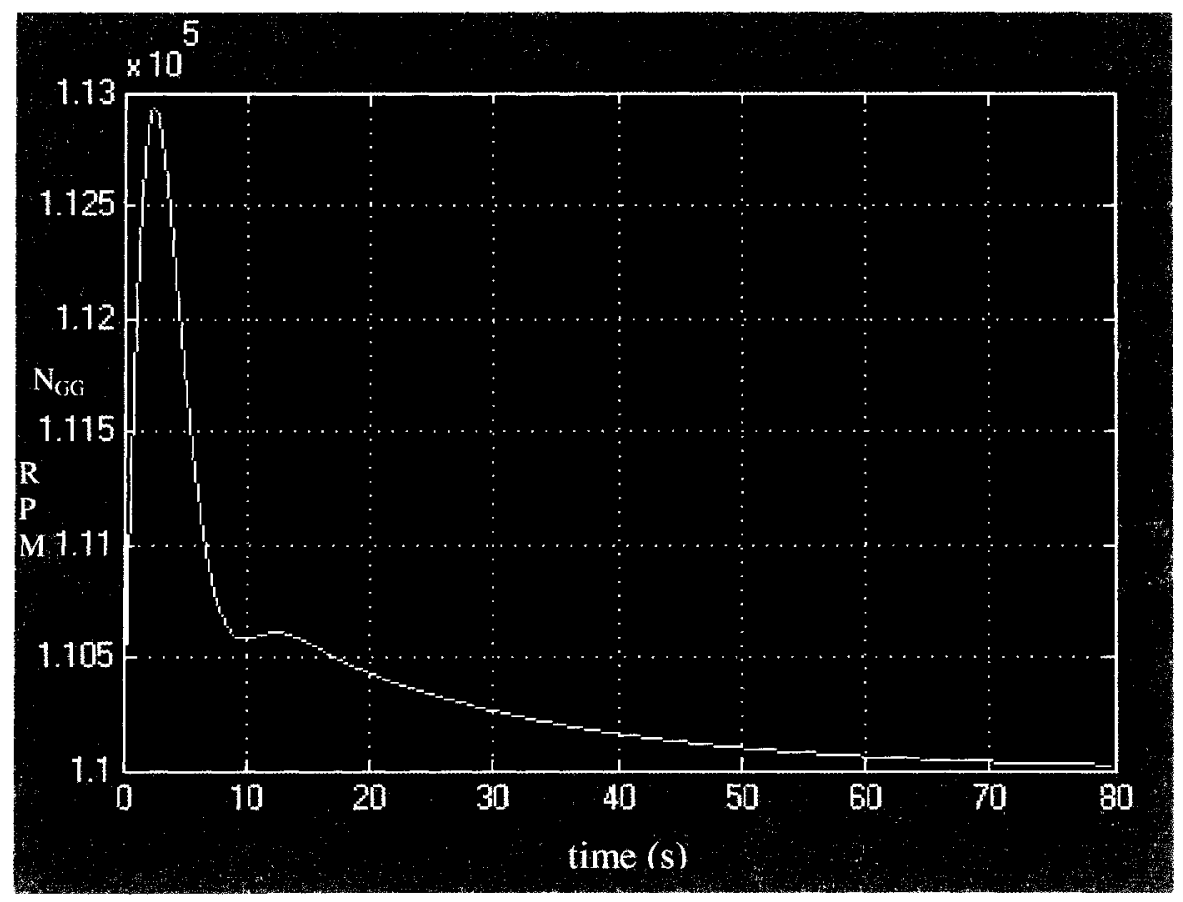

Figure 5-17: PID controller response 


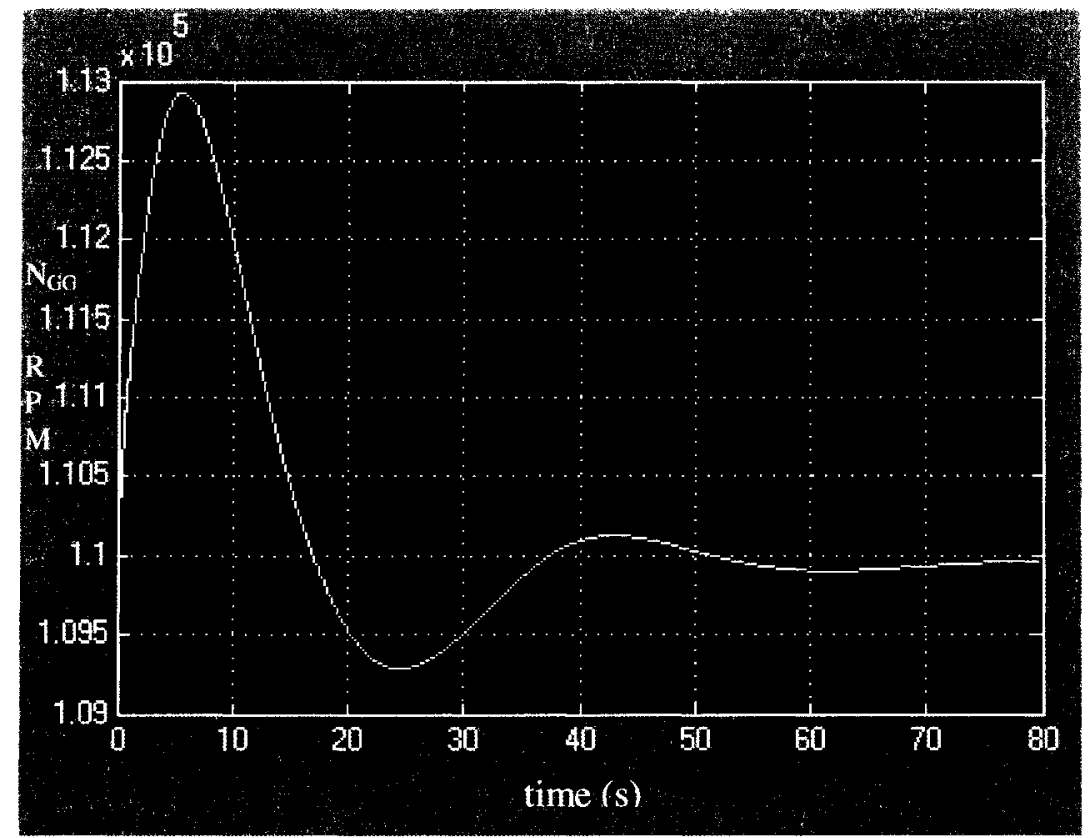

(a)

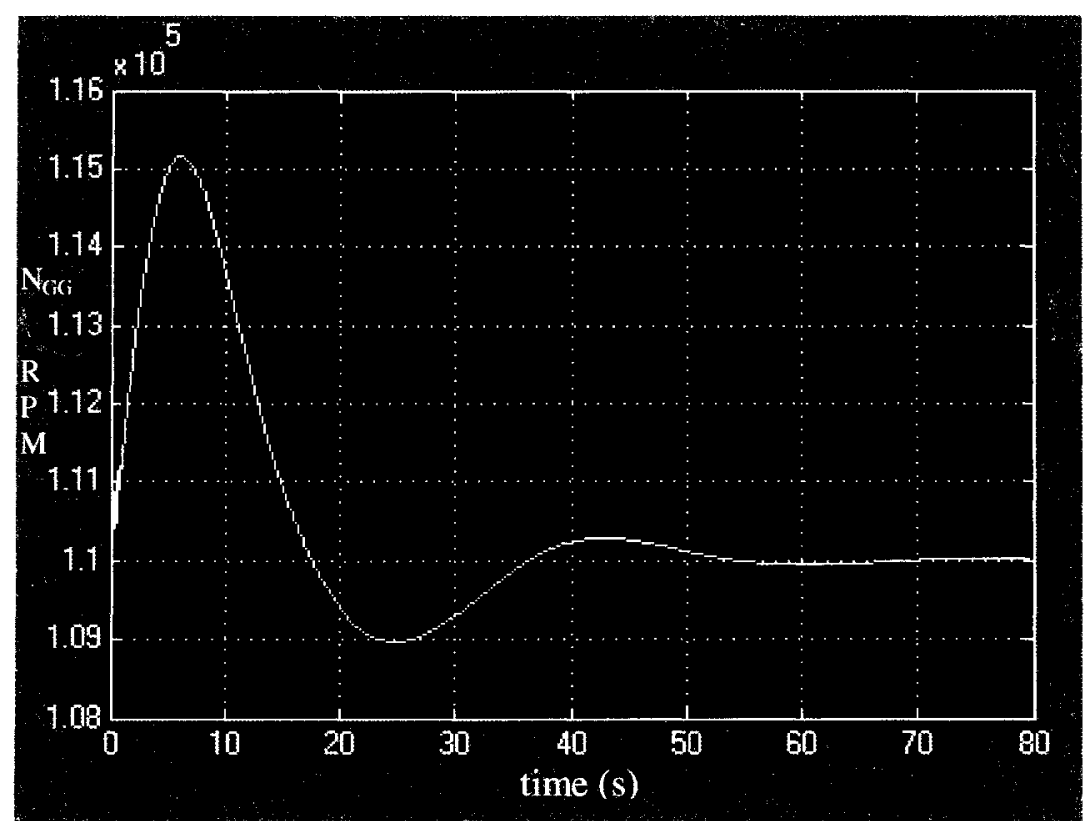

(b)

Figure 5-18: Response of fuzzy logic controller: (a) designed FLC, (b) modified membership functions FLC showing higher overshoot and less settling time $(70 \mathrm{~s})$ 


\subsection{Dynamic system modelling using ANNs}

Dynamic systems refer to systems whose state changes over time. These systems can be represented by a linear or a nonlinear relation depending on the nature of the engineering problem under examination; they are often described by differential equations. However, most real life dynamic processes are nonlinear and are difficult to represent mathematically. It may be fair to note that nonlinear systems in their normal operating ranges could be approximated with linear models. Still the accuracy of the resulting mathematical model is limited and may not be adequate to predict the output of the dynamic system outside a certain operating range. One alternative to the linearization is the development of input output models (empirical models, provided that the input and outputs of a system are available) that include the spline functions, polynomials, and threshold models. These are limited to interpolation operations. Johansen (1997) argued that if the system mechanics are partly known, but not to the extent that a correct mathematical could be developed, it may not be feasible to derive an empirical model. Hence; in the absence of a common template for nonlinear model development, the preference in selecting one model over others depends on the described problem. The structure of a proposed model determines its capability to incorporate the necessary system information.

In control engineering for instance, system identification is outlined as the modelling of an undefined process with the intent of predicting the behaviour of the given process or system. The black-box terminology commonly refers to the method of describing a dynamic system's behaviour using only the available observable data. In 
this regard, Sjöberg (1995) pointed out that not all the data can serve the purpose of the modelling and some of it must be omitted and proposed the following approach: the first step utilizes the past recorded data to map a regression vector; the second step consists of mapping from the regression space to the output space (a typical basis function expansion).

The regression vector method for nonlinear systems, which depends on the kind of nonlinearities in the data, can be typically represented in the form of a nonlinear autoregressive moving average term exogenous variables (NARMAX). The concurrence of such models or its derivatives is still questionable. The nonlinear mapping step that follows relies on the estimation of parameters and their quantities. It may be feasible to start with a great number of parameters and then trim them down to just the most significant using learning and generalization methods, such as weight sharing, weight elimination (pruning algorithms), and regularization (Matlab, 2000). As with any system which is not mathematically formulated, the problem of system identification lurks in the choice of model structure and data extraction. In this thesis, a gas turbine system transient behaviour is modeled according to an artificial neural network by using the observable data generated by GasTurb simulation software.

\subsubsection{Design procedure}

GasTurb was the platform for data generation and collection, and the Matlab neural network toolbox was used for ANN development. The GasTurb turboshaft modelling abilities were employed to construct a dynamic model of a $1 \mathrm{MW}$ twin shaft 
gas turbine with a gas generator speed $\left(\mathrm{N}_{\mathrm{GG}}\right)$ of $28000 \mathrm{rpm}$. This GTE is used by the design team. The information pertaining to different turbine characteristics at various operating states was depicted by the program encoded relations and the most relevant parameters for this work were collected as an input output set. These data were then used to develop the artificial neural network models to emulate the GasTurb simulated model at transient operating conditions. This section will show that relationships defining the thermodynamic process can be duplicated with some accuracy by the ANNs. Two ANNs were built for this purpose: one relating fuel flow to turbine inlet temperature (TIT) along the operating line, and the other relating fuel flow to gas generator speed $\left(\mathrm{N}_{\mathrm{GG}}\right)$ along the operating line.

The first ANN can be useful for temperature limit control and the second ANN can be employed in gas generator speed control. For this purpose, a set of data was generated by GasTurb and copied to Matlab as follows:

From the transient option in GasTurb the data is generated by varying the power lever. $\mathrm{N}_{\mathrm{GG}}$, TIT and fuel flow variables are automatically copied to an Excel spreadsheet. The data are then transferred to Matlab software as a file. An ANN is then built using the Neural Network Toolbox available in Matlab. The ANN is then simulated in Simulink using Gensim, "which generates a Simulimk block to simulate a neural network" (Matlab, 2000). The ANN Simulink model and Matlab codes are included in Appendix E. The details of the procedure are summarized in the following sections. 


\subsubsection{Data collection}

These data were generated using the GasTurb transient operation routine. Totally 1094 data sets were collected at various operating points, at transient operating conditions. It includes the fuel flow, $\mathrm{N}_{\mathrm{GG}}$, and TIT at different operating ranges of the turbine (from $70 \%$ to $100 \% \mathrm{~N}_{\mathrm{GG}}$ ) The reason behind choosing this operating range is that below that range the operating line crosses the surge line and, it is not be feasible to predict the performance of the GTE at that point. The relation between the $\mathrm{N}_{\mathrm{GG}}$, TIT and the fuel flow variables will be regenerated using a feedforward neural network.

\subsubsection{Neural process modelling}

For system modelling, the choice of a signal that is used to represent a typical input to the dynamic system is of some importance. Schoukens (1998) suggests that a nonlinear system should be tested with a multisine signal with amplitude and frequency distribution matching that of a typical input to the system. However, since gas turbine systems are complex and have diverse inputs, it is difficult to define a typical input. In this thesis, the author will use sets of data relating fuel flow to $\mathrm{N}_{\mathrm{GG}}$ produced by varying the demand for power as the input signal. Thus, the artificial neural network will be structured and trained to identify the relation embedded in the thermodynamic model.

For this purpose a number of patterns related to the system are presented to the network as training sets. The proper number of training sets is a factor in the convergence of the network. Techniques to determine the adequate number of the 
training sets from a data set are found in the literature (Haykin, 2001). One of these methods is highlighted in Equations 5-4-1 and 5-4-2.

$$
\begin{gathered}
\psi \geq \frac{32 W}{e} \ln \left(\frac{32 Q}{e}\right) \\
\psi>\frac{W}{e}
\end{gathered}
$$

where $\psi$ is the number of random sets, $W$ is the sum of network weights, $Q$ is the number of hidden neurons, and $e$ is the training error. A data set was generated by varying the input signal (varying the demand for power lever in GasTurb). The set contained 750 training examples for the $\mathrm{N}_{\mathrm{GG}}$ variable, 165 of which were used for validation purposes, and 78 were used for testing. It also comprised 706 learning examples for the TIT variable, 315 of which were used for validation, and 73 were used for testing. The test data were never seen by the ANN during training or validation processes. The artificial neural networks constructed for both variables have the feedforward network structure, with the training method used being the Levenberg-Marquardt, which offered fast convergence.

\subsubsection{ANNs structure}

A feedforward network with one hidden layer of sigmoidal activation functions and one output layer with linear functions was constructed. The reason for choosing this structure is drawn from Cybenco (1989) and Nergraad (2000). Cybenco demonstrated that an ANN with such topology is capable of approximating any nonlinear continuous function through the expansion of that function as a superposition of sigmoidal functions. 
Nergraad underscored the abilities of these feedforward networks to model simple and complex functional relations. The network is characterized by its weights and biases that are determined during the training phase. To make sure the ANNs generalize, the number of hidden neurons was determined by minimizing the sum of square error (cost function) of the validation data set. The network was then trained with different numbers of hidden units and the cost function was calculated after each training session. The network shown in Figure 5-19 with one hidden layer and an optimum number of 9 hidden neurons met the design criteria set by the cost function and given in Equation 5-4-3.

$$
\text { error } \left.=\frac{1}{2 n} \sum[t-a)\right]^{2}
$$

Where $n$ is the number of sample data, $t$ is the system output, and $a$ is the model estimated output. The test error is calculated according to Equation 5-5-1. The results of the simulation are presented next.

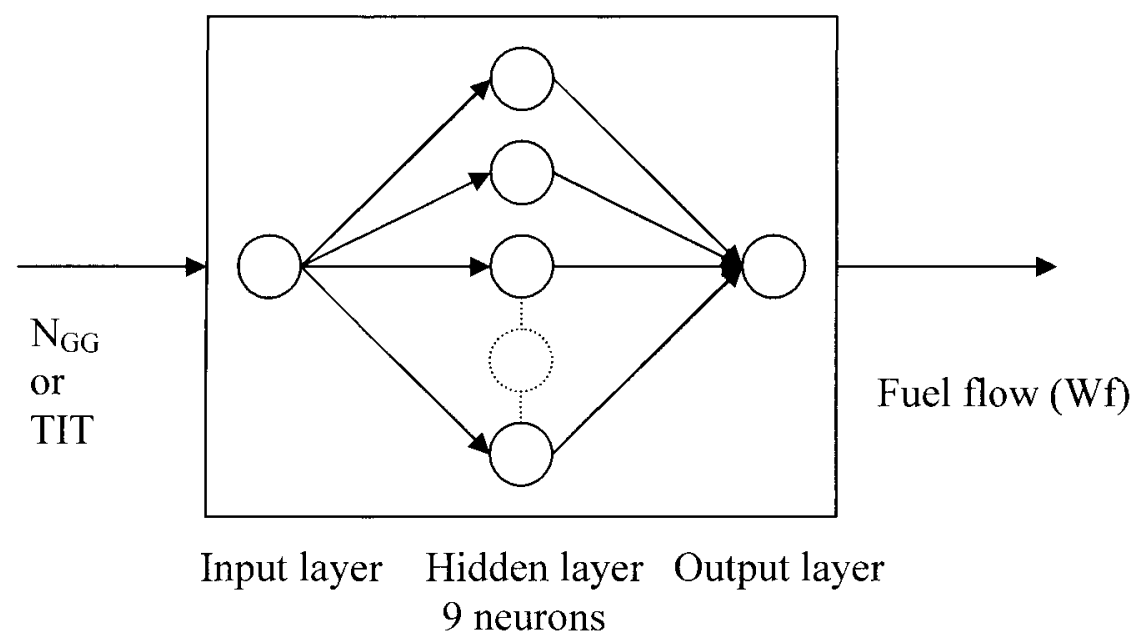

Figure 5-19: Feedforward ANN with one hidden layer containing 9 hidden neurons; the input is $\mathrm{N}_{\mathrm{GG}}$ or TIT and the output is fuel flow 


\subsection{Results}

As mentioned earlier, two ANNs were built (hereafter referred to simply as NNs): one predicting the $\mathrm{N}_{\mathrm{GG}}$, fuel flow relation, and the second duplicating the TIT, fuel flow relation for the transient performance of the $1 \mathrm{MW}$ GTE.

\section{Wf versus $N_{G G} N N$ simulation}

Figure 5-20 shows the NN training output as compared to the GasTurb simulation program for a cycle of 3000 epochs (equivalent to number of iterations). The figure shows a selected range since the complete data cannot be illustrated clearly on one graph.

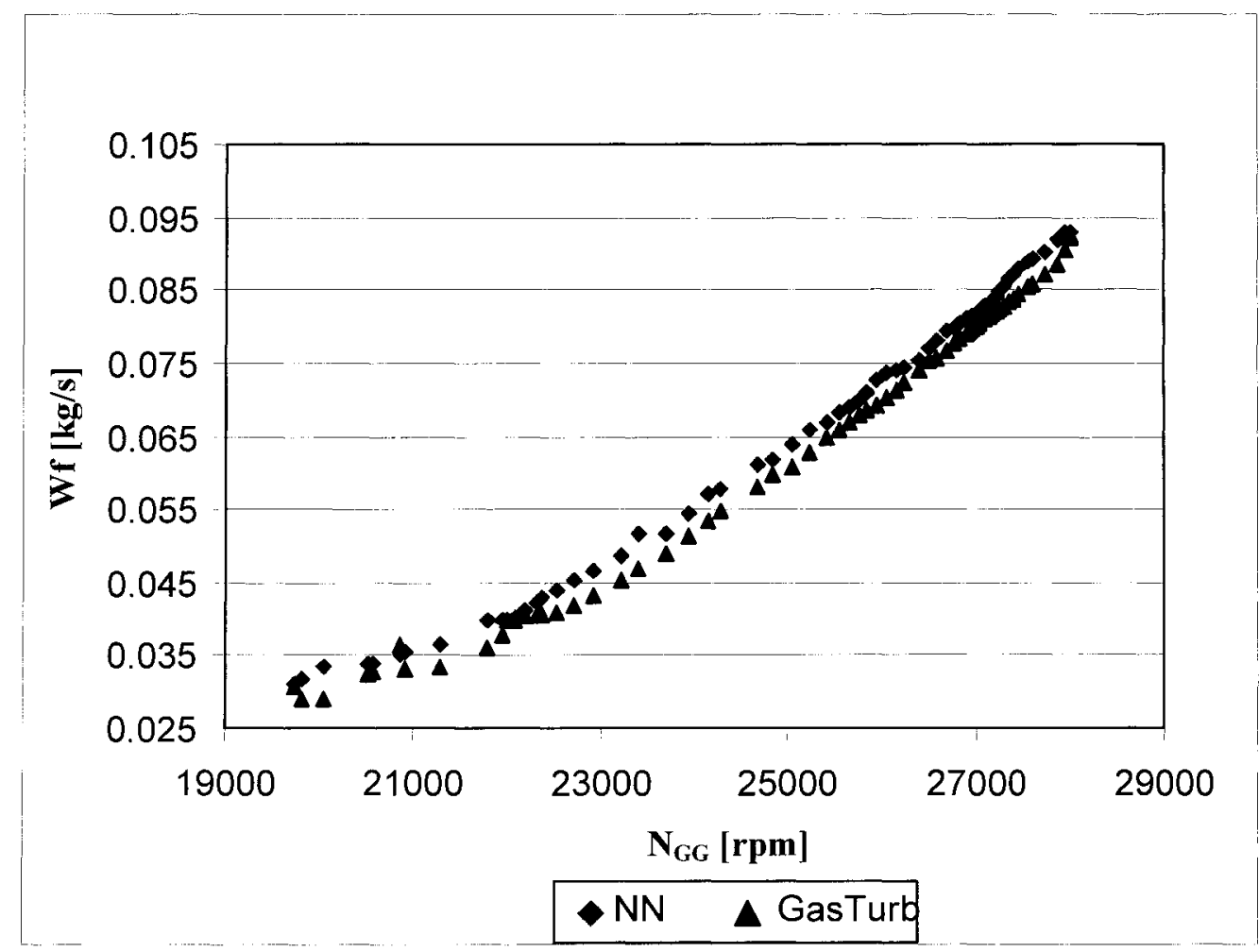

Figure 5-20: NN compared to GasTurb simulation model training data $\left(\mathrm{N}_{\mathrm{GG}}\right)$ 
As can be seen from Figure 5-20, the NN was able to learn from the patterns presented to it. Nonetheless, the performance of the network will be assessed through the validation and test data as shown in Figures 5-21 and 5-22.

Figure 5-21 depicts the $\mathrm{N}_{\mathrm{GG}}$ validation data distribution and Figure 5-22 exhibits the sum of square error distribution. A sum of square error of $1.22 \mathrm{e}-006$ was marked in the case of the $\mathrm{N}_{\mathrm{GG}}$ versus Wf relationship.

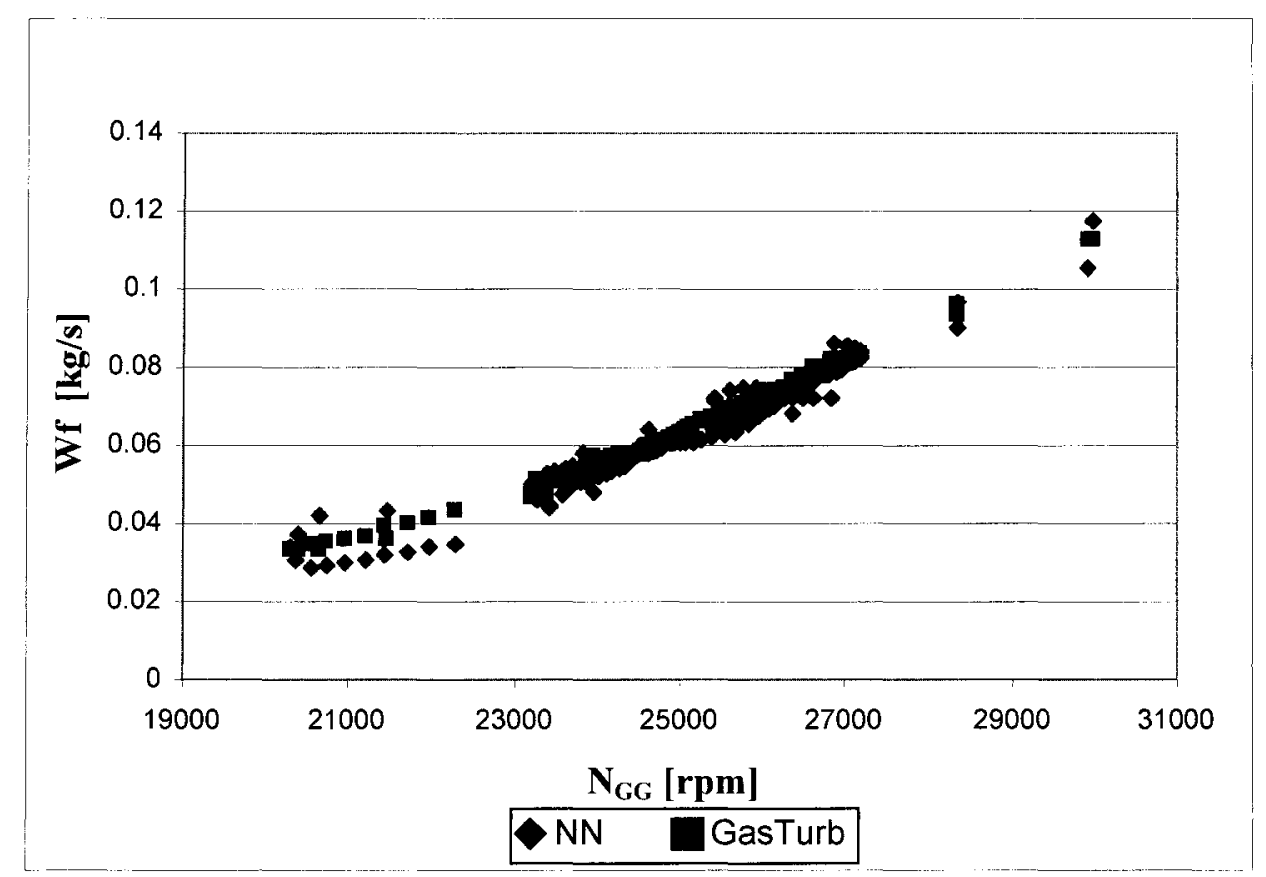

Figure 5-21: $\mathrm{N}_{\mathrm{GG}}$ validation data distribution

The test error for the $\mathrm{N}_{\mathrm{GG}}$ variable is featured in Figure 5-23. It is worth mentioning that the sum of square error for this procedure was found to be $5.72 \mathrm{e}-007$. These results show that the NN has the abilities of depicting the relationship between the fuel flow and $\mathrm{N}_{\mathrm{GG}}$ with some accuracy. The following paragraph will detail the design of a NN to predict the fuel flow versus TIT relationship. 


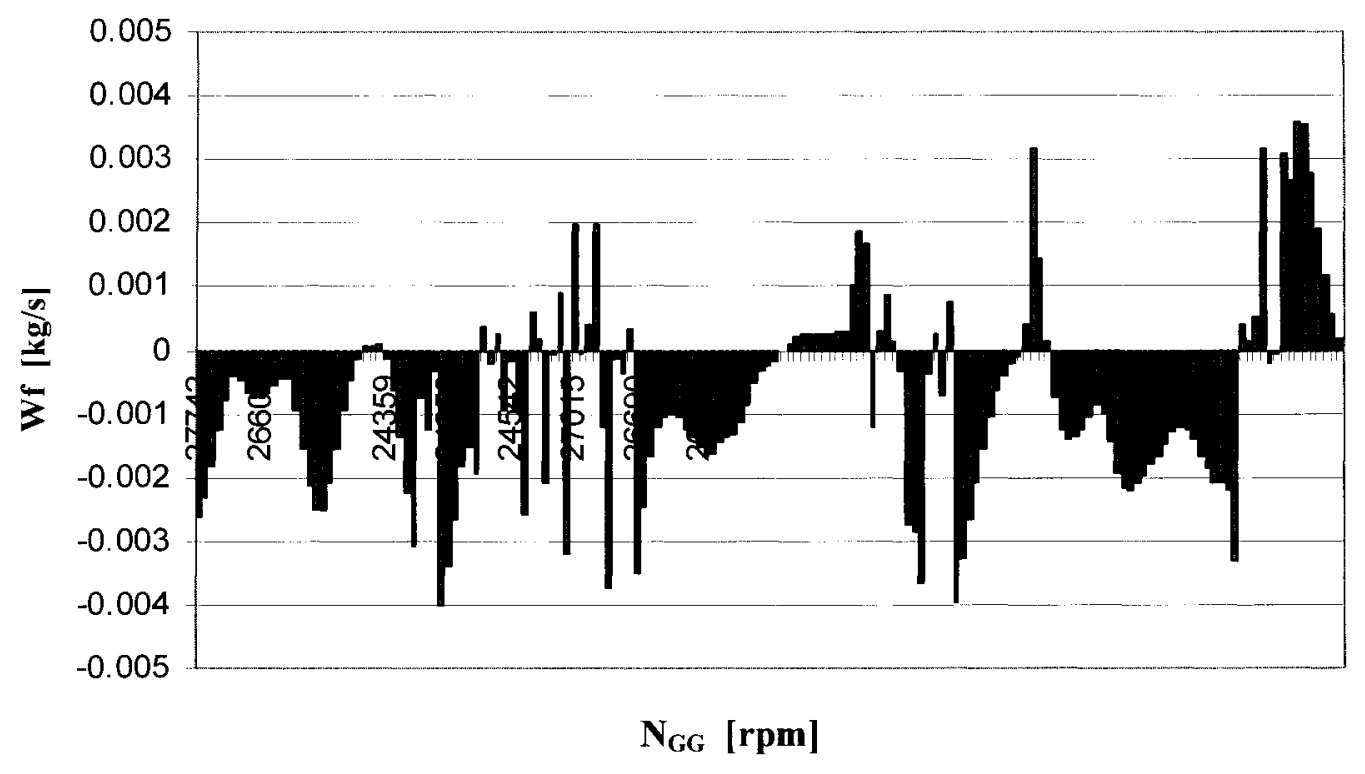

Figure 5-22: $\mathrm{N}_{\mathrm{GG}}$ validation error

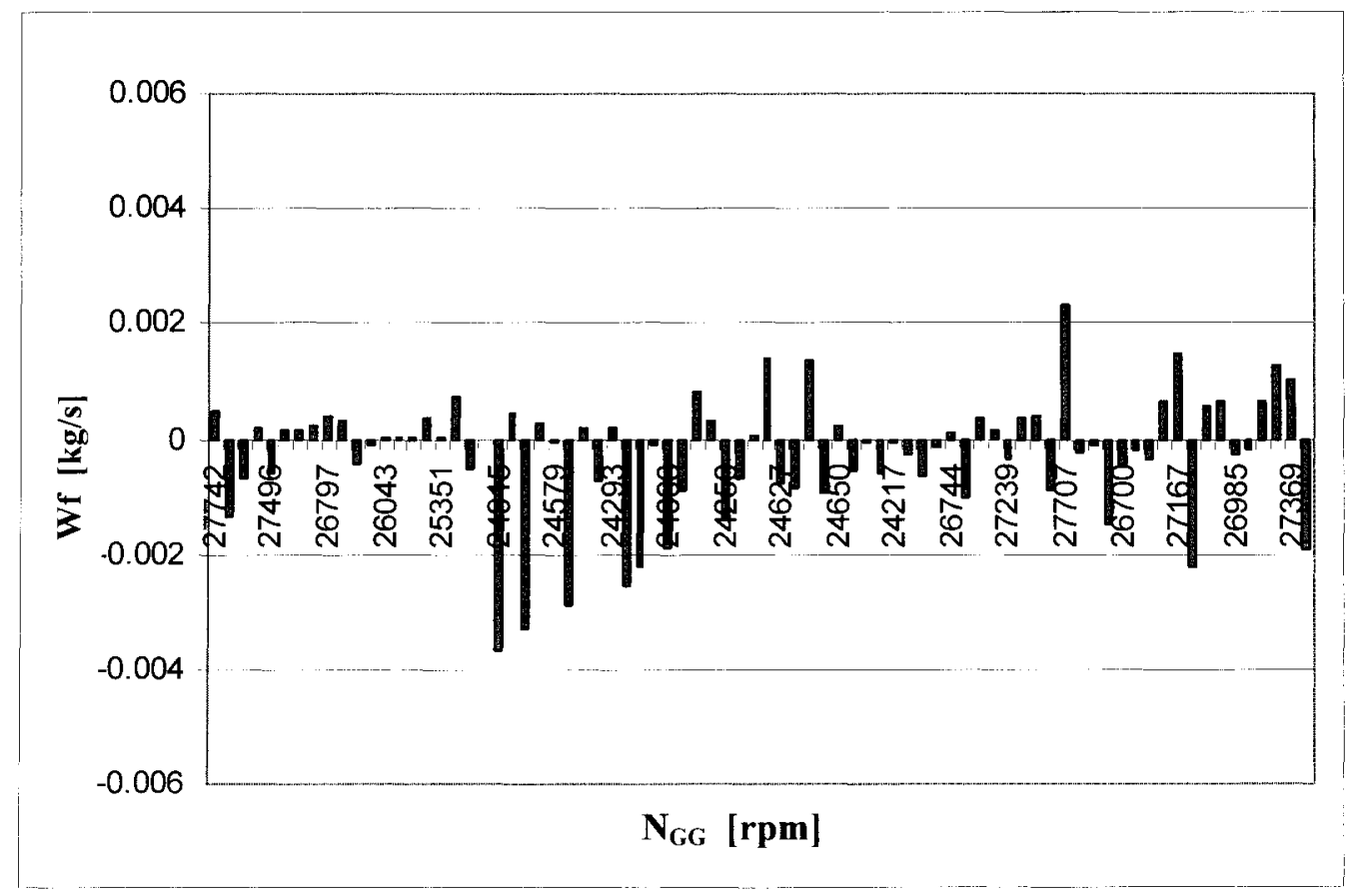

Figure 5-23: $\mathrm{N}_{\mathrm{GG}}$ test error 
The training and test errors based on Equation 5-5-1 (Fine, 1999) are found in Table 5-7.

$$
\mathrm{E}=\frac{1}{n}\left|\frac{a-t}{t_{\max }-t_{\min }}\right|
$$

where $t_{\max }$ and $t_{\min }$ are the maximum and minimum of the data set, and $n$ is the number of sample data.

\section{Wf versus TIT NN simulation}

The same procedure is followed in modelling the TIT versus fuel flow relationship. A feedforward NN with one layer of 9 neurons was designed. Figure 5-24 illustrates the variance between the NN training and the GasTurb model data.

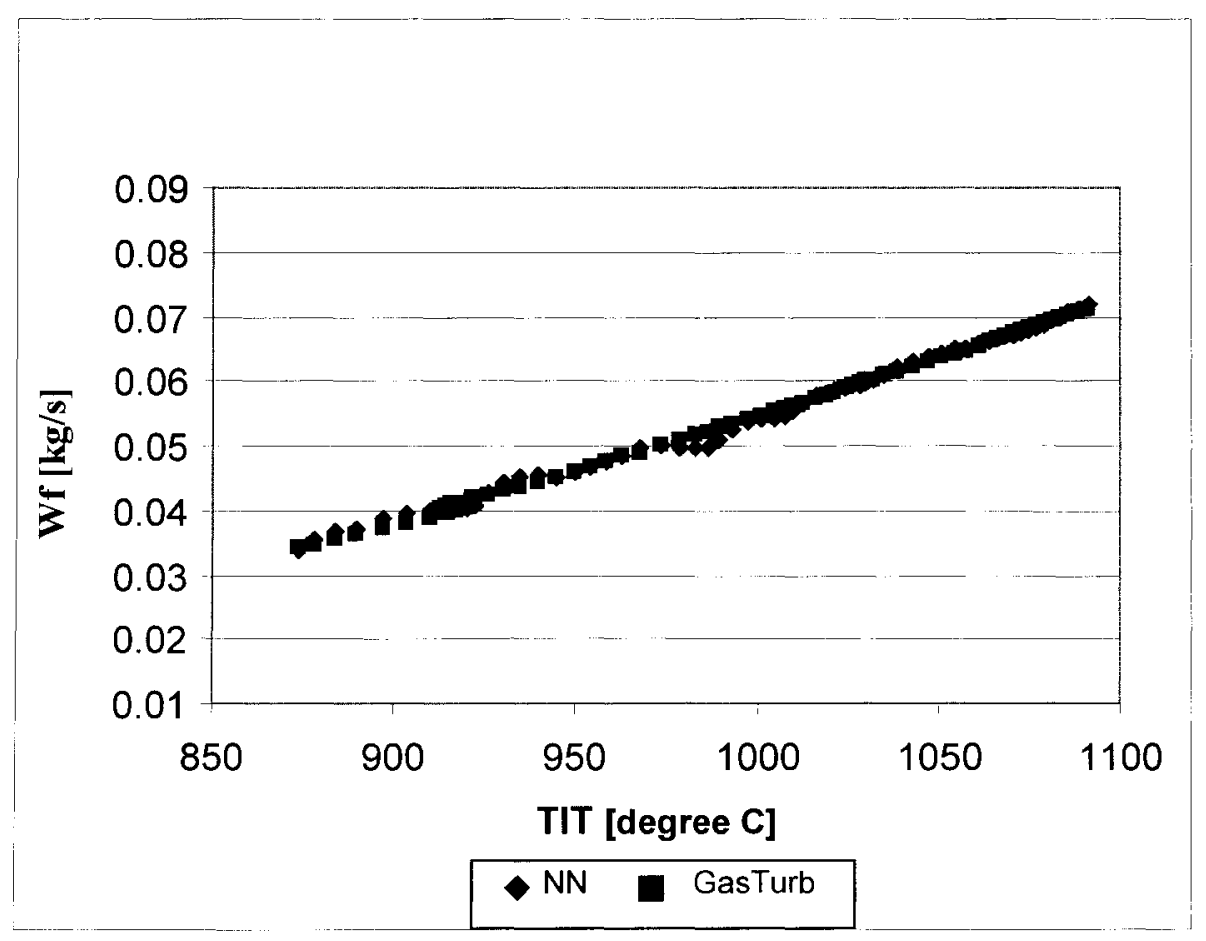

Figure 5-24: NN compared to GasTurb TIT training data variance 
The validation data and error for TIT-fuel flow relation is pictured in Figures 525 and $5-26$, respectively. The sum of square error for the validation data was calculated to be $8.25 \mathrm{e}-007$, while the same error for test data was computed as $3.43 \mathrm{e}-007$. The results for the test data in terms of variance and error are shown in Figures 5-27 and 5-28.

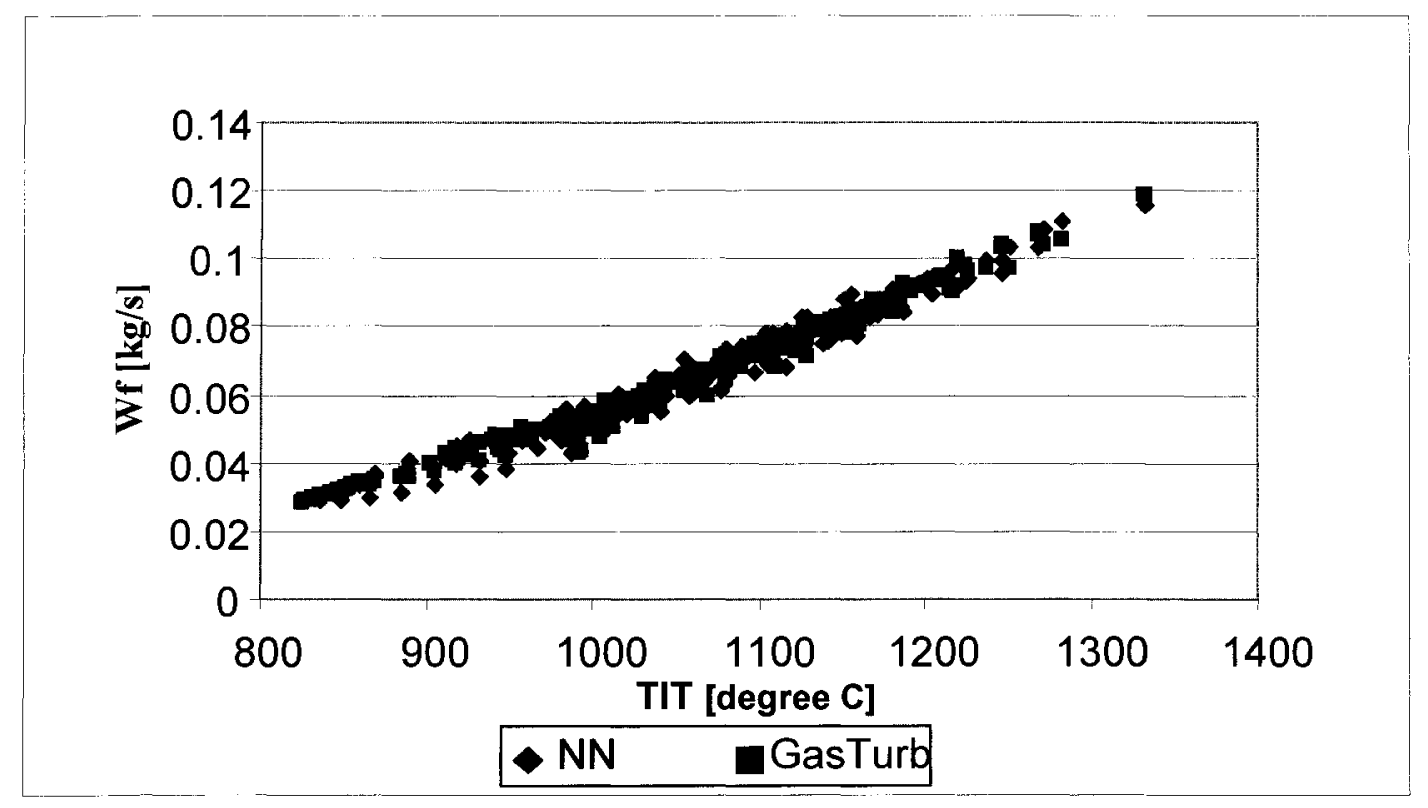

Figure 5-25: TIT validation data 


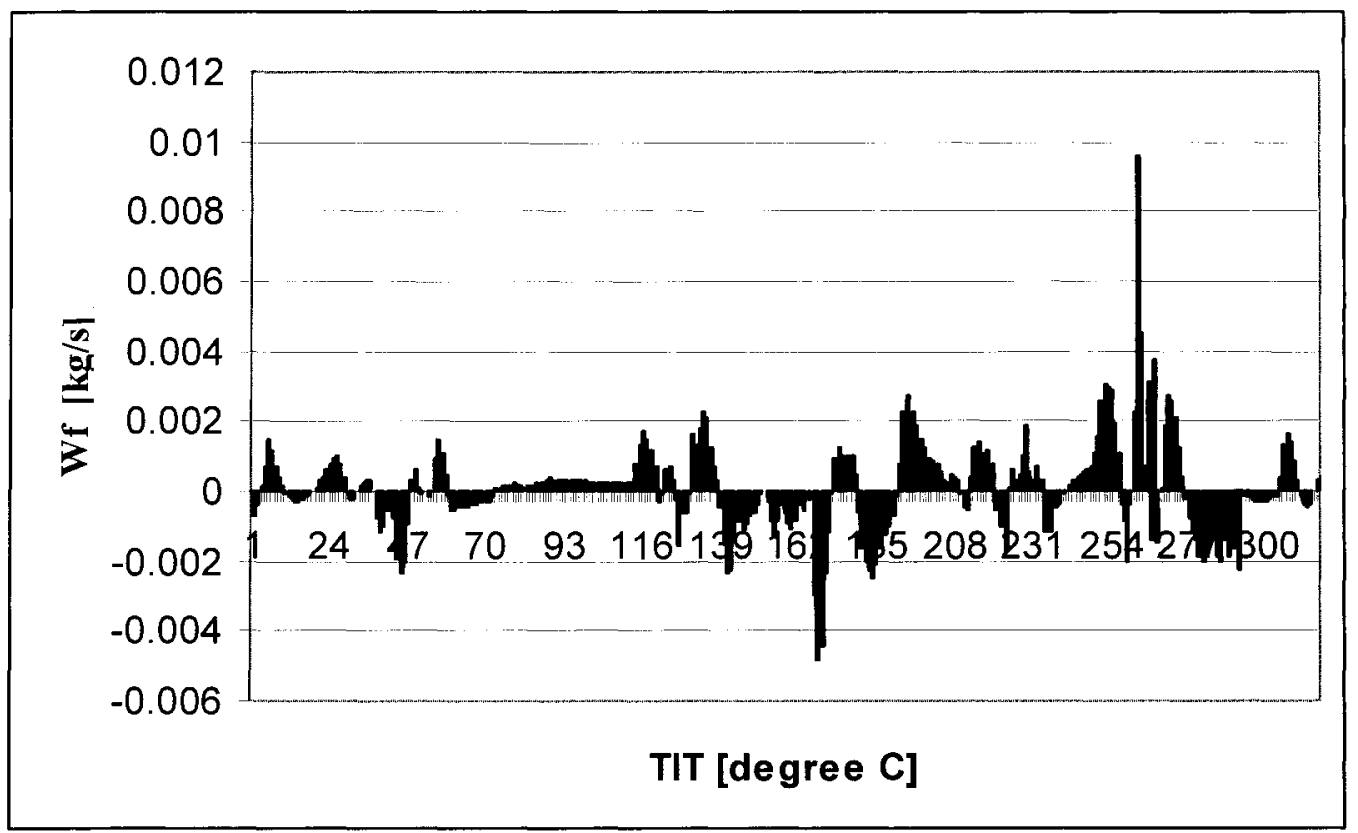

Figure 5-26: TIT validation data error

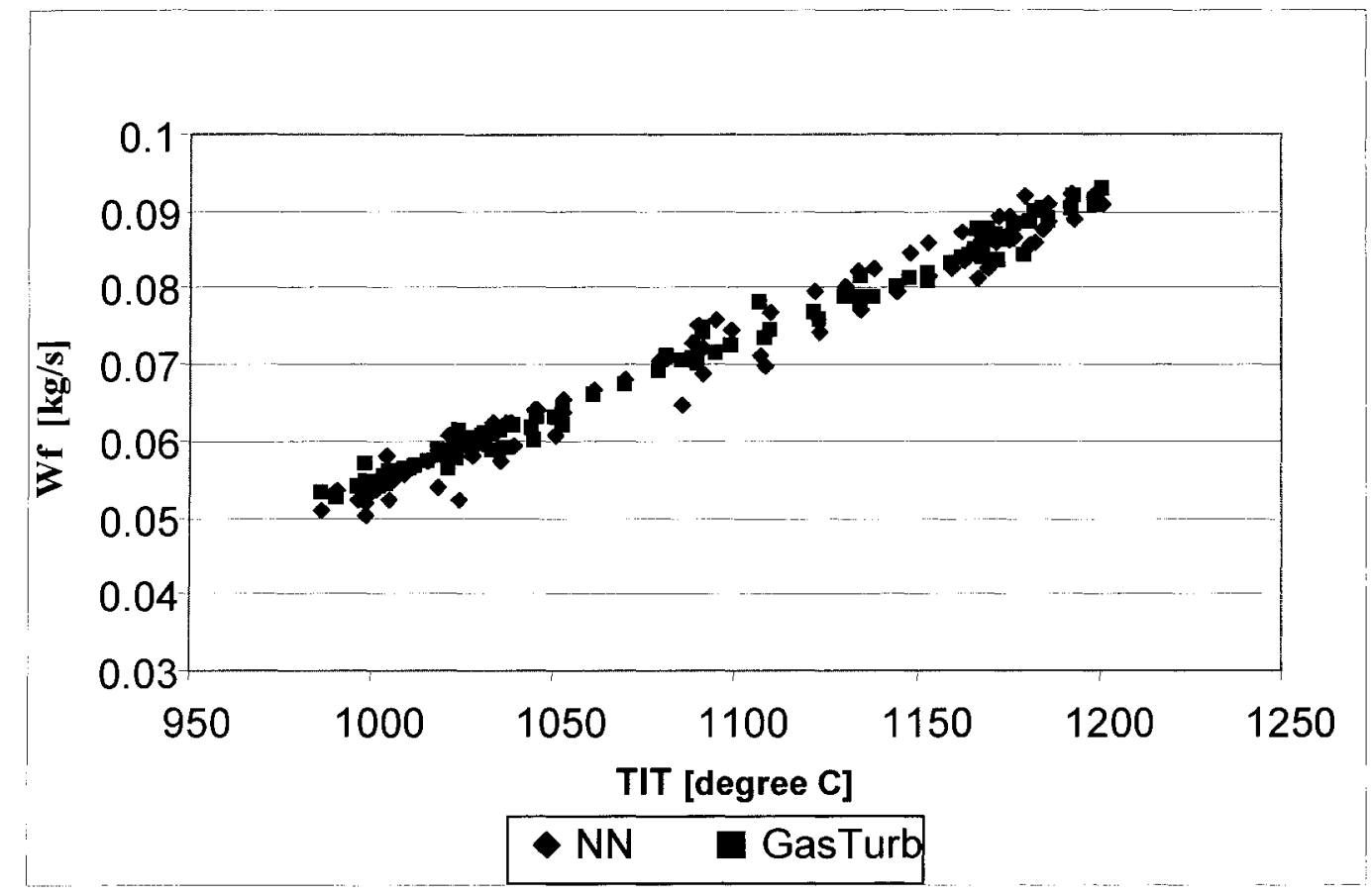

Figure 5-27: TIT test data variance 


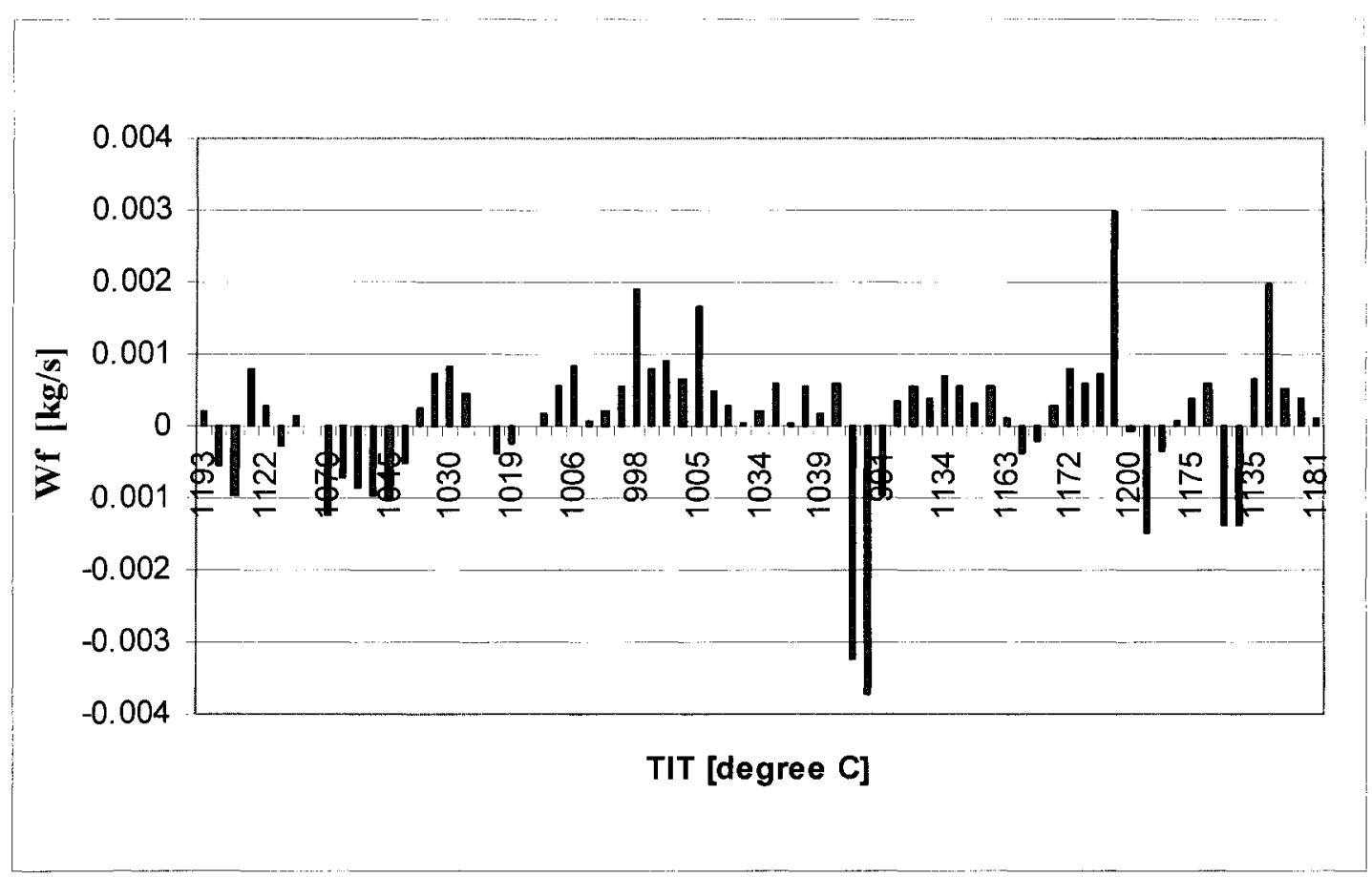

Figure 5-28: TIT test data error

The training and test errors of the TIT and $\mathrm{N}_{\mathrm{GG}}$ neural networks are found in Table 5-8. The error values, as can be seen, are less then $2 \%$. This $2 \%$ value is taken as an indication that the artificial neural networks have been trained properly.

Table 5-8: Training and test error

\begin{tabular}{|l|c|c|}
\hline Error in \% & Wf versus TIT neural & Wf versus GG $_{\text {G }}$ neural \\
$\mathrm{E}=\frac{1}{n}\left|\frac{a-t}{t_{\max }-t_{\min }}\right|$ & network & network \\
\hline Training & 1.33 & 1.35 \\
\hline Test & 1.67 & 1.77 \\
\hline
\end{tabular}


The results produced in this section are satisfactory. This section presented a method based on feedforward ANNs to model the gas turbine engine. The next step would be to combine the modelling and the control by expert knowledge in order to produce a controlled gas turbine engine. However, this will not be covered in this thesis. 


\section{Chapter 6}

\section{Conclusion}

The application of fuzzy logic and artificial neural networks to the control and modelling of gas turbine engines paves the way for a new era in the identification and control of nonlinear dynamic systems. This thesis summarizes two fuzzy logic systems, the Mamdani and the Sugeno models, and proposes fuel schedules and the subsequent control of a gas turbine engine based on these two systems. Both systems were able to approximate functions characterizing the acceleration and deceleration schedules. The proposed fuzzy logic controller performance was assessed for a specified GTE operating conditions and the response due to a PID controller action was shown. The major advantages of FLC systems are the ease of development and implementation.

Artificial neural network modelling is represented in this work as a method for systems identification. The ability of this technique to approximate any nonlinear function, by mapping an input vector to its corresponding output vector makes it useful for modelling dynamic systems from data series. In gas turbine applications, models derived from data in the development phase and validated on test-beds are more effective than thermodynamic models in predicting the engine performance for control purposes. In this thesis, the transient operation of the gas turbine engine was modelled by two-layer feedforward artificial neural networks. The ANNs used were able to emulate the GasTurb relationships between fuel flow and $\mathrm{N}_{\mathrm{GG}}$, and between fuel flow and TIT on the operating line. This work can be extended in the future to encompass the closed-loop 
control of a modelled gas turbine engine at steady state operating conditions. 


\section{References}

Albus J.S., A New Approach to Manipulator Control, the Cerebellar Model Articulation Controller, Transaction ASME, Journal Dynamic System, Measurement Control, Pages 220-227, September 1975.

Andrew C., Peter F., and Hugh B., Edited by Lakhim C Jain., N.M. Martin, Fusion of Neural Networks, Fuzzy Sets, and Genetic Algorithms, Industrial Applications, CRC Press, Pages 297-348, 1999.

Athans M., Kapasouries P., Kappos E., and Spang H. A., Linear-Quadratic Gaussian with Loop-Transfer Recovery Methodology for the F-100 Engine. Journal of Guidance, Control, and Dynamics, Volume 1, Pages 45-52, 1986.

Balakrisnan S.R., Mishra S.K., Sundararjan V., and Damodaran K.A., Fuzzy Computing for Control of Aero Gas Turbine Engines, Defense Science Journal, Volume 44, No. 4, Pages 295-304, October 1994.

Blotenberg W., An Advanced Control and Monitoring System for Turbo machinery, Asme, International Gas Turbine and Aeroengine Congress and Exposition, Huston, Texas, June 5-8, 1995.

Boyce M., Gas Turbine Engineering Handbook, Second Edition, ButterworthHeinemann, Huston, Texas, 2002.

Berton J., (NASA), Ultra Efficient Technology Program, Glenn Research Center, 2004.

Buckley J.J., On the Equivalence of Neural Nets and Fuzzy Expert Systems, Fuzzy Sets and Systems, Volume 53, Pages 129-134, 1993.

Carpenter G.A., Grossberg S., and Rosen D.B., Fuzzy Art, Fast Stable Learning and Categorization of Analog Pattern by an Adaptive Resonance System. Neural Networks, Volume 4, No. 6, Pages 759-772, 1991.

Chen C.H., Fuzzy Logic \& Neural Network Handbook, IEEE Press, McGraw Hill, NY., 1999.

Chi-Hua W., Yun-Hua X., and Ben-Wei L., Application of Fuzzy Controller in the Fuel System of a Turbojet Engine, Journal of Propulsion and Power, Volume 5, No.3, Pages 373-374, 1989. 
Chiras N., Evans C., and Rees D., Nonlinear Gas Turbine Modeling Using Feedforward Neural Networks, Proceedings of ASME Turbo Expo 2002, Amsterdam, The Netherlands, June 3-6, 2002.

Cohen H., Rogers G.F.C., and Saravanamuttoo H.I.H., Gas Turbine Theory, Longman Scientific \& Technical, John Wiley \& Sons, Inc., NY, 1987.

Cybenco G., Approximation by Superposition of a Sigmoidal Function in Mathematics of Control, Signals and Systems. Springer-Verlag, NY, Volume 2, Pages 303-314, 1989.

Dayhoff J., Neural Network Architectures, Van Nostrand Reinhold, NY, 1990.

DeHoff R.L., Hall W.E., Adams R.J., and Gupta N.K., F100 multivariable Control Synthesis Program: Development of F100 Control System, Systems Control, Inc. Palo Alto, Calif., AFAPL-TR-77-35, Volume 1, June 1977

Delara-Jayme S., Ramirez R.G., Cueves L.C., Reyes C. M., Feedforward Neurofuzzy Speed Control for a Turbogas Unit, Technology Update, Volume 424, Pages 225$235,2002$.

Döhler S., and Rüschendorf L., An Approximation Result for Nets in Functional Estimation, Statistics Probability Letters, 2000.

Evans C., Fleming P.J., Norton J.P., Pratt I., Rees D., and Rodriguez V.K., Application of System Identification Techniques to Aircraft Gas Turbine Engines, Control Engineering Practice, No. 9, Pages 135-148, 2001.

Fine L., Feedforward Neural Network Methodology, Springer-Verlag, NY, 1999.

Fortuna L., Muscato G., Nunnari G., and Occipinti L., Neural Modeling and Fuzzy Control: Regulation of the Temperature of an Oven, Springer-Verlag, London, 1995.

Frederick D.K., Garg S., and Adibhalta S., Turbofan Engine Control Design Using Robust Multivariable Control Technologies, IEEE Transactions on Control Systems Technology, Volume 11, Pages 961-970, 2000.

Garg S., Robust Integrated Flight Propulsion Control Design for a STOVL Aircraft Using H-Infinity Control Design Techniques, Automatica, Volume 1, Pages 129-145, 1993.

GasTOPS, Course Notes, Carleton University, Ottawa, Canada, 1990. 
GasTurb, GasTurb Simulation Software, version 10, 2004.

Giampaolo T., The Gas Turbine Handbook: Principles and Practices, Prentice Hall PTR, Upper Saddle River, NJ, 1997.

Hall L., A Comparison of Neural Network and Fuzzy Clustering Techniques in Segmenting Magnetic Resonance Images of the Brain, IEEE Transaction Neural Networks, Volume 3, Pages 672-682, September 1992.

Harefores M., Application of H-Infinity Robust Control to the RM12 Jet Engine, Control Engineering Practice, Volume 9, Pages 1189-1201, 1997.

Haykin S., and Kosko B., Intelligent Signal Processing, IEEE Press, USA, 2001.

Haykin S., Neural Networks, a Comprehensive Foundation, IEEE Press, USA, 1993.

Hebb D.O., The Organization of Behavior, Wiley, NY, 1949.

Hopfield J.J., Neural Networks and Physical Systems with Emergent Collective Computational Abilities, Proceedings National Academic of Science, April 1982, Volume 79, Pages 2554-2558, 1982.

Hopfield J.J., Neurons with Graded Response Have Collective Computational Properties Like Those of Two-State Neurons, in Proceedings National Academic of Science, Volume 81, Pages 3088-3092, May 1984.

Hornik K., Stinchcombe M., and White H., Multilayer Feedforward Networks are Universal Approximators, Neural Networks, Volume 2, Pages 359-366, 1989.

Hueneke K., Jet Engines, Fundamentals of Theory, Design and Operation, Motorbooks International Publishers \& Wholesalers, Osceola, USA, 1997.

Hung W.W., Dynamic-Simulation of Gas Turbine Generating Unit, Proceedings of Institute of Electrical Engineers, Volume 4, 1991.

Jager R., Fuzzy Logic in Control, Techniche Universities Delft, Ph.D Thesis, 1995.

Kaur D., Lin B., On the Design of Neural-Fuzzy Control System, International Journal of Intelligent Systems, Volume 13, Pages 11-26, 1998.

Khan E., Neural Fuzzy Based Intelligent Systems and Applications, Fusion of Neural Networks, Fuzzy Sets, and Genetic Algorithms, Chapter 5, Pages 105-140, 1999.

Klir G., and Yaun B., Fuzzy Sets and Fuzzy Logic; Theory and Applications Prentice 
Hall, NJ, 1995.

Ljung L., Development of System Identification. Proceedings of the $13^{\text {th }}$ IFAC World Congress, San Francisco, USA, Pages 141-146, 1996.

Lyantsev O.D., Breikin T.V., Kulikov G.G., and Arkov V.Y., Optimal Multi-Variable Control of Gas Turbine Engines, International Journal of Systems Sciences, Volume 35, No. 2, Pages 79 - 86, 2004.

MacIsaac B.D., Gas Turbine Performance and Design Supplementary Course Notes, Carleton University, Ottawa, Canada, 1986.

Mamdani E.H., and Assilian S., An Experiment in Linguistic Synthesis with a Fuzzy Logic Controller, International Journal of Man-Machine Studies, Volume 7, No.1, Pages 1-13, 1975.

Matlab Neural Network Toolbox, Version 4, The MathWorks, Inc., Natick, 2000.

Matsumoto H., Ohsawa Y., Takahasi S., Akiyama T., Hanaoka H., and Ishiguro O., Start up Optimization of a Combined Power Plant Based on Cooperative Fuzzy Reasoning and a Neural Network, IEEE Transactions on Energy Conversion, Volume 12, No. 1, March 1997.

McCulloh W., and Pitts W., A Logical Calculus of the Ideas Immanent in Nervous Activity, Bulletin of Mathematical Biophysics 5, Pages 115-133, 1943.

McMorran P. D., Design of Gas Turbine Controllers Using Inverse Nyquist Array Method, Proceedings of Institute of Electrical Engineers, Volume 117, Pages 2050-2056, 1970.

Norgraad M., Raven O., Poulsen N.K., and Hansen L.K., Neural Networks for Modeling and Control of Dynamic Systems, Springer-Verlag, London, UK, 2000.

Otto E. W., and Taylor B. L., Dynamics of Turbojet Engine Considered as Quasi-Static System, NACA Report 1011, 1950.

Pedersen M.W., Hansen L.K., and Larsen J., Pruning with Generalization Based Weight Saliences, $\gamma \mathrm{OBD}, \gamma \mathrm{OBS}$, Proceedings of the Neural Information Processing Systems, Volume 8, 1995.

Pedrycz W., Fuzzy Sets Engineering, CRC Press, Boca Raton, Fla., 1995.

Petriu E., Neural Networks and Fuzzy Logic Systems, Course Notes ELG 5196, University of Ottawa, 2004. 
Pilidis P., and MacCallum N.R.L., The Effect of Heat Transfer on Gas Turbine

Transients. ASME Gas Turbine Conference, Paper 86-GT-275, Pages 1-10, 1986.

Procyk T.J., and Mamdani E.H., A Linguistic Self Organising Process Controller, Automatica, Volume 15, Pages 15-30, 1979.

Rao S.S., and Kumthekar B., A Composite Neural Architecture and Algorithm for Nonlinear System Identification, Intelligent Engineering Systems Through Artificial Neural Networks, Volume 5, Pages 77-84, 1995.

Sanjay G., Propulsion Control Technology Development in the U.S.-A Historic Perspective, International Society of Air Breathing Engines, Ohio, September 2003.

Sanjay G., Controls and Health Management Technologies for Intelligent Aerospace Propulsion Systems, AIAA-2004-0949, NASA / TM Glenn Research Center, 2004.

Saravanamuttoo H.I.H., Overview on Basis and Use of Performance Prediction Methods. AGARD Lecture Series No. 183- Steady and Transient Performance Prediction of Gas Turbine Engine, Paper 1, Pages 1-18, 1992.

Saravanamuttoo H.I.H., Rogers G.F.C., and Cohen L., Gas Turbine Theory, $5^{\text {th }}$ Edition, New York, Prentice Hall, 2001.

Schram G., Kaymak U., and Verbruggen H.B., Fuzzy logic Control, In Robust Flight Control: A Design Challenge, Magni J.F., and Benani S., Eds. Springer-Verlag, London, U.K, Pages 135-147, 1997.

Shoukens J., and Dobrowiecki T., Design of Broadband Excitation Signals with a User Imposed Power Spectrum and Amplitude Distribution, IEEE Instrumentation and Measurement Technology Conference, Pages 1002-1005, 1989.

Simpson P.K., Artificial Neural Systems, Pergamon Press, Elmsford, NY, 1990.

Sjöberg J., and Ljung L., Overtraining, Regularization, and Searching for Minimum in Neural Networks, Preprint IFAC Symposium on Adaptive Systems in Control and Signal Processing, Grenoble, France, Pages 669-647, 1992.

Sjöberg J., Hjalmerson H., and Ljung L., Neural Networks in System Identification, Preprints $10^{\text {th }}$ IFAC Symposium on SYSID, Copenhagen, Denmark. Volume 2, Pages 49-71, 1994. 
snecma Technical Report, snecma group, Every, France, 2005.

Sun G., Dagli C.H., Tammano A., Dynamic Neuro-Fuzzy Control of the Nonlinear Process," Computer Ind. Engng, Volume 33, No. 1-2, Pages 413-416, 1997.

Takagi T., and Sugeno M., Fuzzy Identification of Systems and its Application to Modeling and Control, IEEE Transaction Systems, Man, and Cybernetics, Volume 15, Pages 116-132, 1985.

West J., Best and Tailor's Physiological Basis of Medical Practice, Eleventh Edition, Williams \& Wilkins Pubs., Baltimore, MD., 1985.

Woodward J., Marine Gas Turbines, ISBN 0471 95962-6, Wiley-Interscience Publication, NY, 1975.

Zadeh L.A., Fuzzy Sets, Information Control, Volume 8, Pages 338-358, June 1965.

Zadeh L.A., Fuzzy Logic, IEEE Computer Magazine, Pages 83-93, 1988.

Zadeh L.A., Outline of a New Approach to the Analysis of Complex Systems and Decision Processes, IEEE Transanction Systems, Man, and Cybernetics, Volume SMC-3, Pages 28-44, 1973.

Zengqi S., and Zhidong D., A Fuzzy Neural Network and its Application to Controls, Artificial Intelligence in Engineering, Volume10, Pages 311-315, 1996.

Zero-Emission GT, Simulation Model, Carleton University, 2003. 


\section{Appendix A}

Fuzzy Logic Example (Mamdani -type inference engine) 


\section{Fuzzy logic example with two inputs - one output variables.}

This example will cover the fuzzy process for two input variables (TIT and $\mathrm{N}_{\mathrm{GG}}$ ) and one output variable (Wf).

\section{System design}

Four steps are followed:

Step1: Identification of the system's inputs, fuzzy ranges and degree of membership functions $(\mu)$ (Fuzzification).

Step 2: Identification of the system's outputs, fuzzy ranges and degree of membership functions.

Step 3: Identification of the rules that map the inputs to the outputs (rule-base and inference system).

Step 4: Selection of the method that combines the fuzzy rules into a crisp system output (defuzzification).

\section{$\underline{\text { Step 1: }}$}

Input variables fuzzification and degree of membership functions are shown in Figure A1.
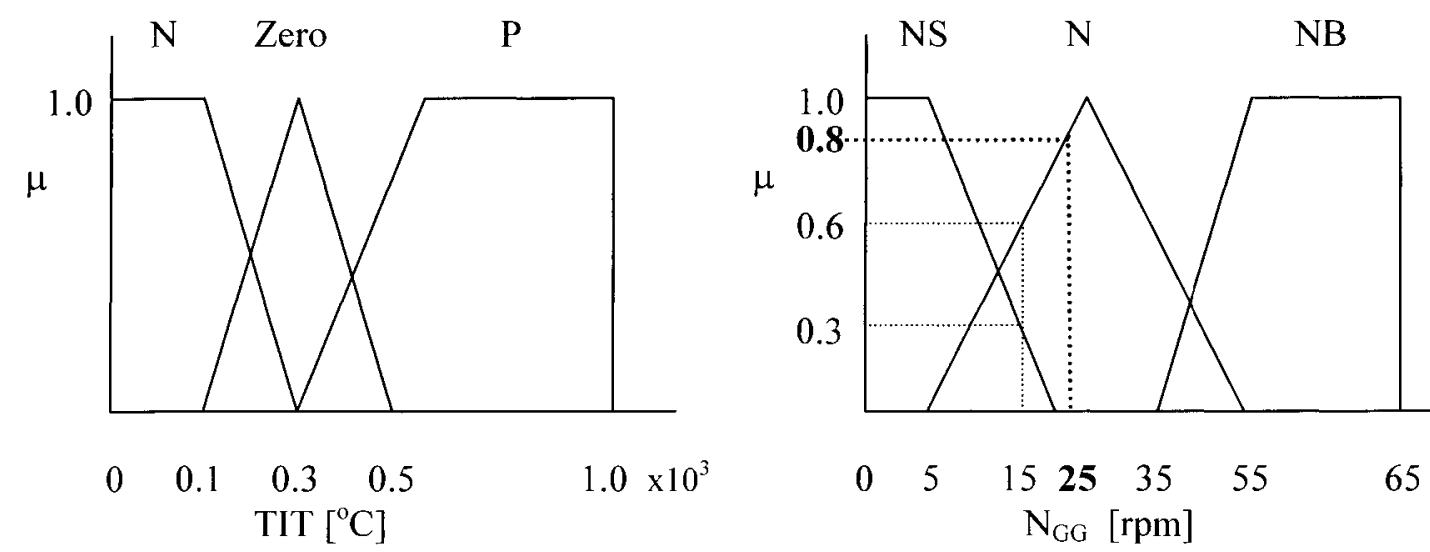

Figure A-1: The degree of membership functions for the two inputs: TIT and $\mathrm{N}_{\mathrm{GG}}$ 
The range of $\mathrm{N}_{\mathrm{GG}}$ is [ $\left[\begin{array}{ll}0 & 65\end{array}\right]$ with three linguistic values each represented by a fuzzy set. The linguistic values for $\mathrm{N}_{\mathrm{GG}}$ are: $\mathrm{NS}, \mathrm{N}$, and NB.

TIT input has a range of $\left[\begin{array}{ll}0 & 10^{3}\end{array}\right]$ and is represented by three linguistic values: N, Zero, and $\mathrm{P}$.

The linguistic values have no quantitative significance. To associate a value to them we refer to the membership functions representing them. Each linguistic value has a fuzzy set. The shape of the fuzzy set determines its degree of membership. For example, an $\mathrm{N}_{\mathrm{GG}}$ input of $25 \mathrm{rpm}$ has a degree of membership of 0.8 in the $\mathrm{N}$ fuzzy set, and an input of $15 \mathrm{rpm}$ has a degree of membership of 0.3 in the NS fuzzy set and 0.6 in the N fuzzy set (as shown in Figure A-1).

\section{Step 2:}

Defining output variables (Wf) membership functions as per Figure A-2.

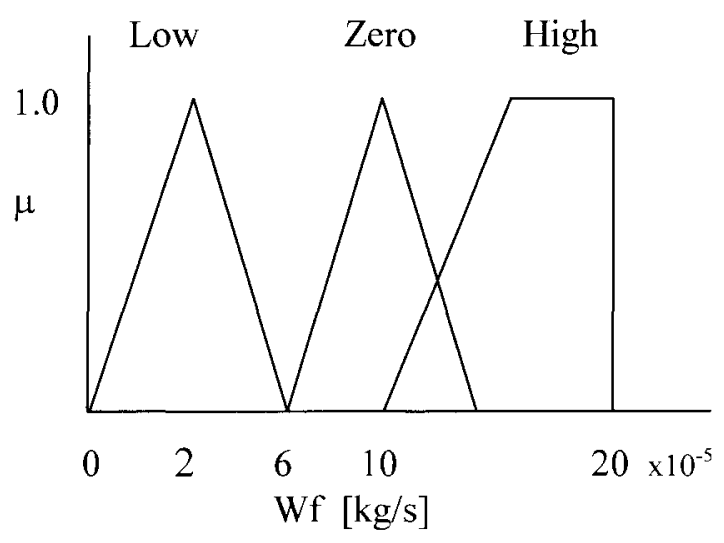

Figure A-2: The membership functions of output variable: Wf 
Step 3:

Rule base

The rule base definition is done to map each input to a corresponding output. One way is to use the linguistic representation of the rules:

$$
\text { If }<\text { antecedent }>\text { then }<\text { consequent }>
$$

For example,

If $<$ TIT is $\mathrm{N}$ and $\mathrm{N}_{\mathrm{GG}}$ is NS $>$ then $<\mathrm{Wf}$ is Zero $>$.

The "and" operation is a fuzzy operation designating the minimum value of the membership functions for selection (or the rule firing strength). I.e., if the membership function of TIT corresponding to $\mathrm{N}$ is less then the membership function of $\mathrm{N}_{\mathrm{GG}}$ corresponding to NS, then the TIT value is selected.

In our example all nine input condition combination must be mapped to an output. This can be represented in a matrix form as follows (Figure A-3):

\begin{tabular}{|c|c|c|c|}
\hline \multirow{2}{*}{\multicolumn{2}{|c|}{$(\mathrm{TIT}) \stackrel{\left(\mathrm{N}_{\mathrm{GG}}\right)}{\longrightarrow} \mathrm{N}$}} & & \\
\hline & & $\mathrm{N}$ & NB \\
\hline$N$ & Zero & Zero & High \\
\hline Zero & Low & Low & Zero \\
\hline$P$ & Low & Low & Low \\
\hline
\end{tabular}

Figure A-3: The rule-base represented in a matrix form

Two rules out of the nine rules are presented below in their linguistic form: 
If $<$ TIT is $\mathrm{P}$ and $\mathrm{N}_{\mathrm{GG}}$ is $\mathrm{NS}>$ then $<\mathrm{Wf}$ is Low $>$ (Rule 1$)$

If $<$ TIT is $\mathrm{P}$ and $\mathrm{N}_{\mathrm{GG}}$ is $\mathrm{N}>$ then $<\mathrm{Wf}$ is Low $>$ (Rule 2)

To incorporate these rules and the rule firing strength into our example, let's consider the two input variables TIT and $\mathrm{N}_{\mathrm{GG}}$ to be 0.51 and 15 consecutively as shown in Figure A-4.
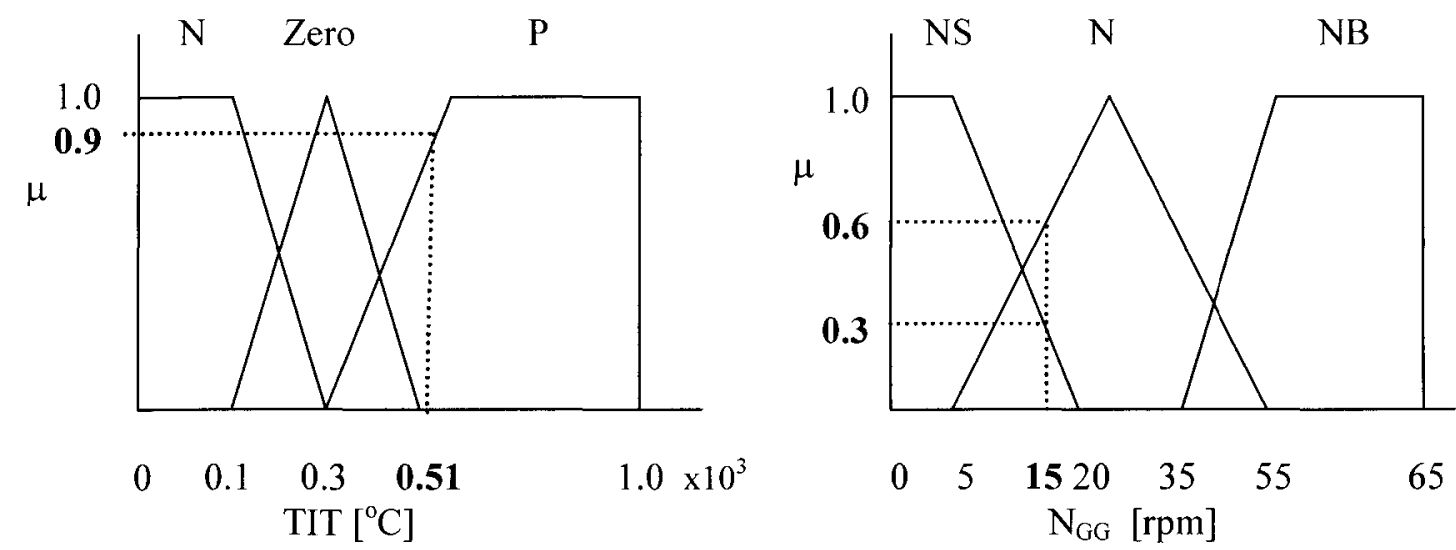

Figure A-4: Inputs representation

The rule firing strength for each rule (Rule1 and Rule 2) are depicted in Figure A-5

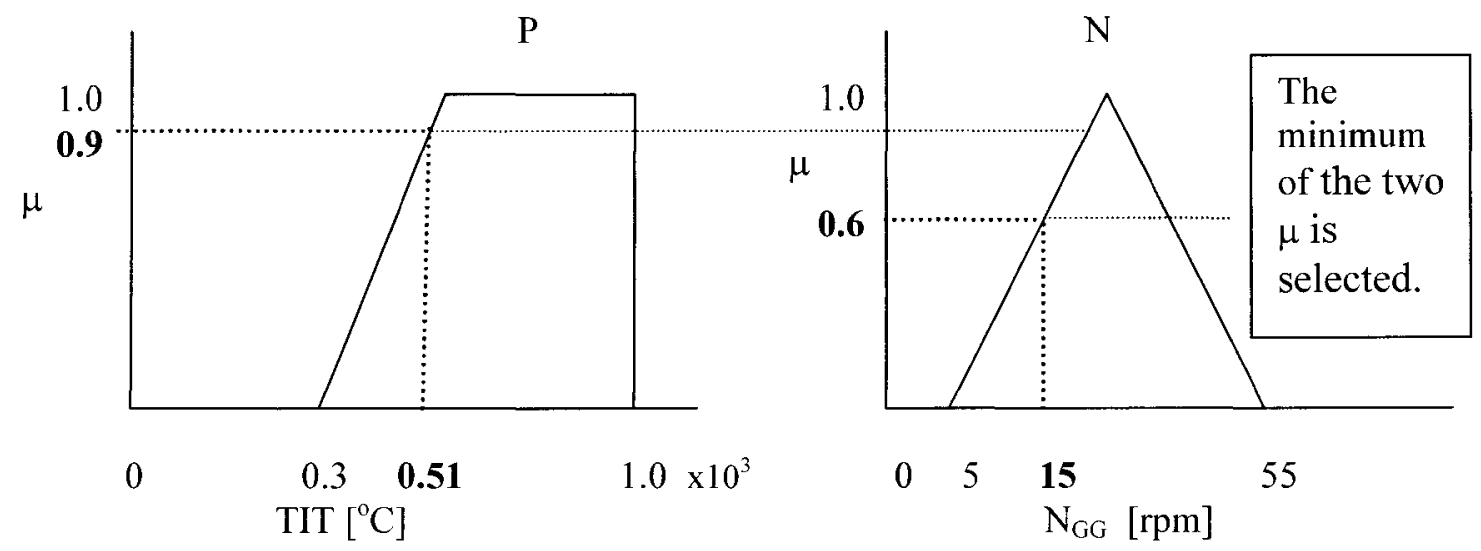

(a) 


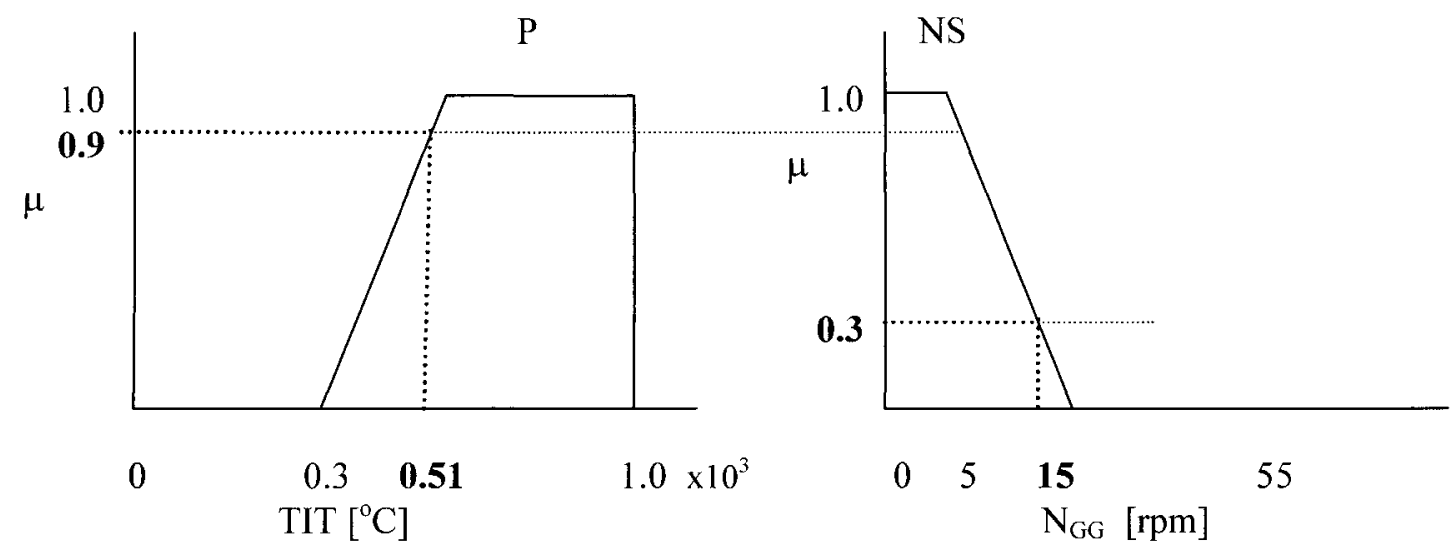

(b)

Figure A-5: Rule firing strength; (a) for Rule 2 it is 0.6 and (b) for Rule 1 it is 0.3 After determining the rules firing strength, the consequent are evaluated using the fuzzy inference system (engine) as follows:

\section{Mamdani fuzzy inference system}

Figure A-6 illustrates how these rules are translated into crisp output in the fuzzy inference system using the minimum operator (as implication function). The centroid of area method is used.

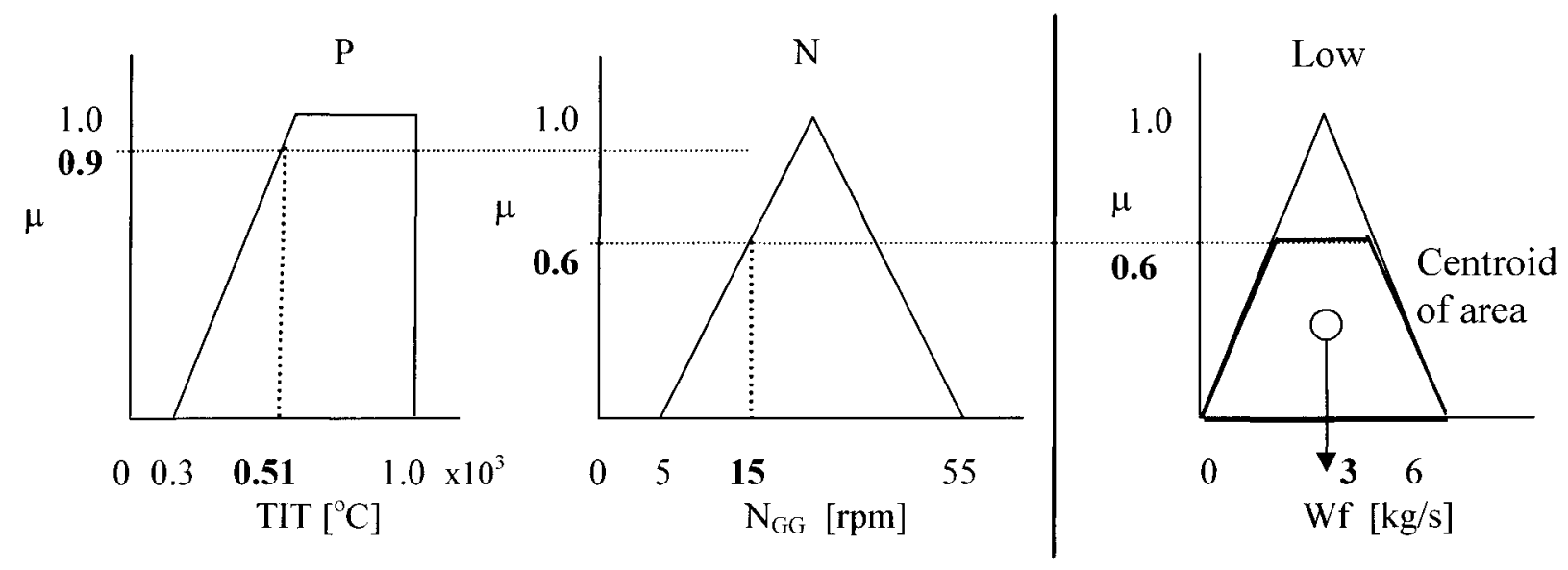

(a) 


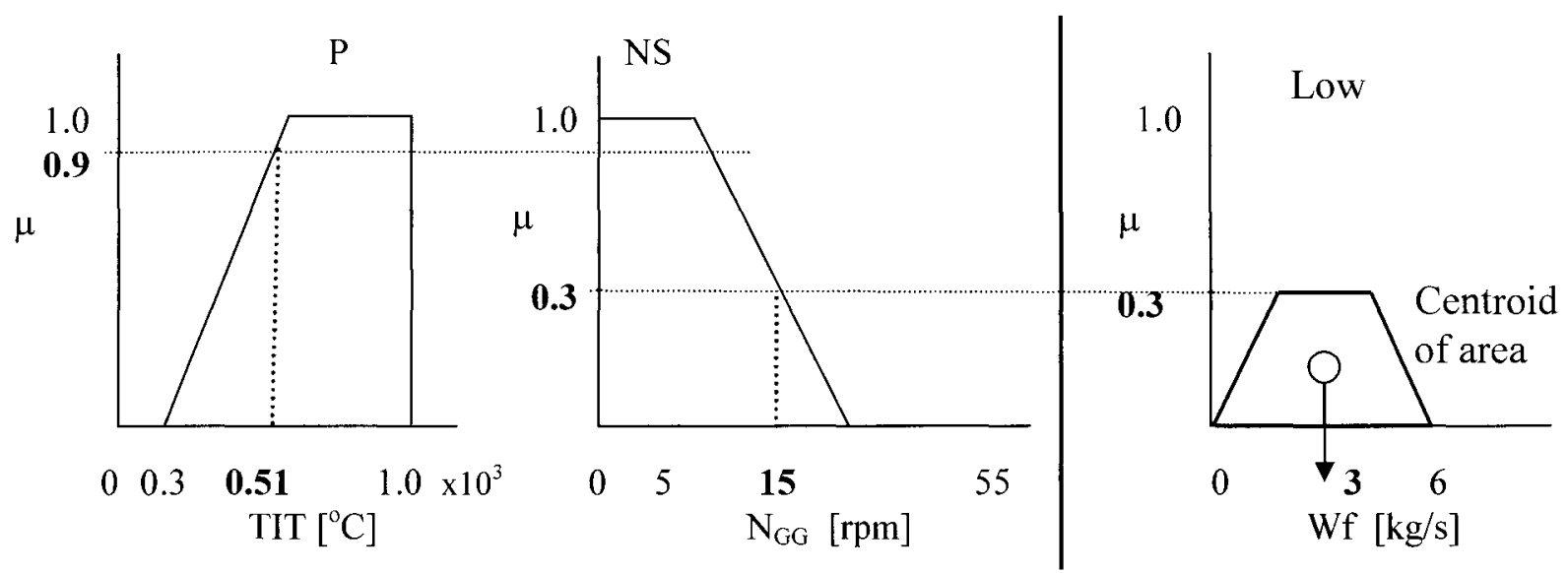

(b)

Figure A-6: Fuzzy inference system with centroid of area method; (a) Rule 2 crisp output calculation and (b) Rule 1 crisp output, both outputs are 3

Step 4:

The last step in the fuzzy process is the defuzzification (the combination of all consequent crisp values into a single crisp output). In this example the weighted average method is used as follows:

$$
\begin{gathered}
C=\frac{\sum_{k=1}^{M} \mu(\text { output }) \cdot \text { output }}{\sum_{k=1}^{M} \mu(\text { output })} \quad M \text { is the number of rules. } \\
C=\frac{\mu_{\text {Rule } 1} *(\text { Centroidofarea } 1)+\mu_{\text {Rule } 2} *(\text { centroidofarea } 2)}{\mu_{\text {Rule } 1}+\mu_{\text {Rule } 2}} \\
C=\frac{0.3 * 3+0.6 * 3}{0.3+0.6} \\
C=3
\end{gathered}
$$

or

$$
\mathrm{Wf}=3[\mathrm{~kg} / \mathrm{s}]
$$




\section{Appendix B}

GasTurb Data for Fuel Schedule

B-1 


\section{GasTurb data for acceleration and deceleration schedules of a 1 MW GTE}

The data included in the worksheet below was generated by GasTurb simulation software. The input data included in Subsection 5.1.3 and the procedures described in Section 5.1 are used to produce the output $\mathrm{Wf} / \mathrm{P}_{3}$ and $\mathrm{GG}$ speed $\left(\mathrm{N}_{\mathrm{GG}}\right)$. The different values for these two variables are included in the worksheet. The most "relevant variables" are: the Surge $\mathrm{Wf} / \mathrm{P}_{3}$, the operating $\mathrm{Wf} / \mathrm{P}_{3}$, the acceleration schedule (Acc.sch), and the deceleration schedule (Dec.sch). The included inlet turbine temperature (T4), mass flowrate (W2), pressure ratio (PR), and compressor efficiency (comp.eff) were useful in the calculation of the relevant variables, e.g., the Acc.sch. and the Dec.sch. These two schedules were produced using the fuzzy logic control approach as described in Chapter 5. 
GasTurb data for fuzzy logic controller (fuel schedules worksheet)

P3 : $\quad$ Compressor exit pressure $[\mathrm{kPa}]$

Wf : $\quad$ Fuel flow $[\mathrm{Kg} / \mathrm{s}]$

T4: Inlet turbine temperature TIT [Degree C]

PR: $\quad$ Compressor pressure ratio

comp.eff: Compressor efficiency

Acc.sch.: Wf $\quad$ W 3 on acceleration schedule $[\mathrm{kg} / \mathrm{s} / \mathrm{kPa}]$ (GasTurb data)

Dec.sch.: Wf $\quad$ W 3 on deceleration schedule $[\mathrm{kg} / \mathrm{s} / \mathrm{kPa}]$ (GasTurb data)

Operating line: $\mathrm{Wf} / \mathrm{P} 3$ on operating line $[\mathrm{kg} / \mathrm{s} / \mathrm{s}]$

W2: Air mass flowrate [kg/s]

GG speed: Gas turbine rotational speed

Thermodynamic design variables

\begin{tabular}{|r|c|c|c|c|}
\hline GG speed & T4 & W2 & PR & comp.eff \\
\hline 27983.4 & 1658.7 & 5.3 & 9.40 & 0.695 \\
\hline 27267.5 & 1667.2 & 5.2 & 9.01 & 0.694 \\
\hline 26593.7 & 1675.7 & 4.9 & 8.60 & 0.69 \\
\hline 25898.7 & 1697.3 & 4.6 & 8.08 & 0.685 \\
\hline 25163.5 & 1570.6 & 4.2 & 7.32 & 0.675 \\
\hline 24468.1 & 1627.1 & 3.8 & 6.64 & 0.665 \\
\hline 23754 & 1496.1 & 3.5 & 5.95 & 0.658 \\
\hline 22974.5 & 1535.1 & 3.3 & 5.52 & 0.645 \\
\hline 22638.8 & 1452.1 & 2.9 & 4.78 & 0.637 \\
\hline 21691.8 & 1421.3 & 2.7 & 4.31 & 0.62 \\
\hline
\end{tabular}

Surge fuel flow ratio

\begin{tabular}{|c|r|r|}
\hline Wf & \multicolumn{1}{|c|}{$\mathbf{P}_{3}$} & \multicolumn{1}{|c|}{ Surge Wf/P $\mathbf{3}$} \\
\hline 0.1580 & 942.725 & 0.000167599 \\
\hline 0.1550 & 904.355 & 0.00017136 \\
\hline 0.1491 & 863.199 & 0.000172695 \\
\hline 0.1424 & 811.08 & 0.000175524 \\
\hline 0.1272 & 734.334 & 0.000173153 \\
\hline 0.1150 & 665.81 & 0.000172737 \\
\hline 0.0999 & 597.299 & 0.000167245 \\
\hline 0.0909 & 553.434 & 0.000164229 \\
\hline 0.0749 & 479.43 & 0.000156185 \\
\hline 0.0666 & 432.82 & 0.000153921 \\
\hline
\end{tabular}

Fuel schedules (acceleration \& deceleration schedules)

\begin{tabular}{|c|r|r|r|r|r|}
\hline Wf & \multicolumn{1}{|c|}{ P3 } & Operating line & GG speed & Acc.sch. at midway & Dec.sch. at 20\% \\
\hline 0.0926 & 802.6 & 0.000115392 & 27983.4 & 0.000141496 & $9.23138 \mathrm{E}-05$ \\
\hline 0.0847 & 761.8 & 0.000111172 & 27267.5 & 0.000141266 & $8.89373 \mathrm{E}-05$ \\
\hline 0.0773 & 719.8 & 0.000107441 & 26593.7 & 0.000140068 & $8.59526 \mathrm{E}-05$ \\
\hline 0.0707 & 679.3 & 0.00010411 & 25898.7 & 0.000139817 & $8.32879 \mathrm{E}-05$ \\
\hline 0.0645 & 638.4 & 0.000101074 & 25163.5 & 0.000137114 & $8.08595 \mathrm{E}-05$ \\
\hline 0.0583 & 595.6 & $9.79387 \mathrm{E}-05$ & 24468.1 & 0.000135338 & $7.83509 \mathrm{E}-05$ \\
\hline 0.0525 & 552.2 & $9.51601 \mathrm{E}-05$ & 23754 & 0.000131202 & $7.61281 \mathrm{E}-05$ \\
\hline 0.0470 & 507.7 & $9.26096 \mathrm{E}-05$ & 22974.5 & 0.000128419 & $7.40877 \mathrm{E}-05$ \\
\hline 0.0421 & 464.4 & $9.07372 \mathrm{E}-05$ & 22638.8 & 0.000123461 & $7.25898 \mathrm{E}-05$ \\
\hline 0.0383 & 424.2 & $9.02149 \mathrm{E}-05$ & 21691.8 & 0.000122068 & $7.21719 \mathrm{E}-05$ \\
\hline
\end{tabular}


Appendix C

Simulink and Matlab Codes for 1 MW GTE Fuel Schedules

C-1 


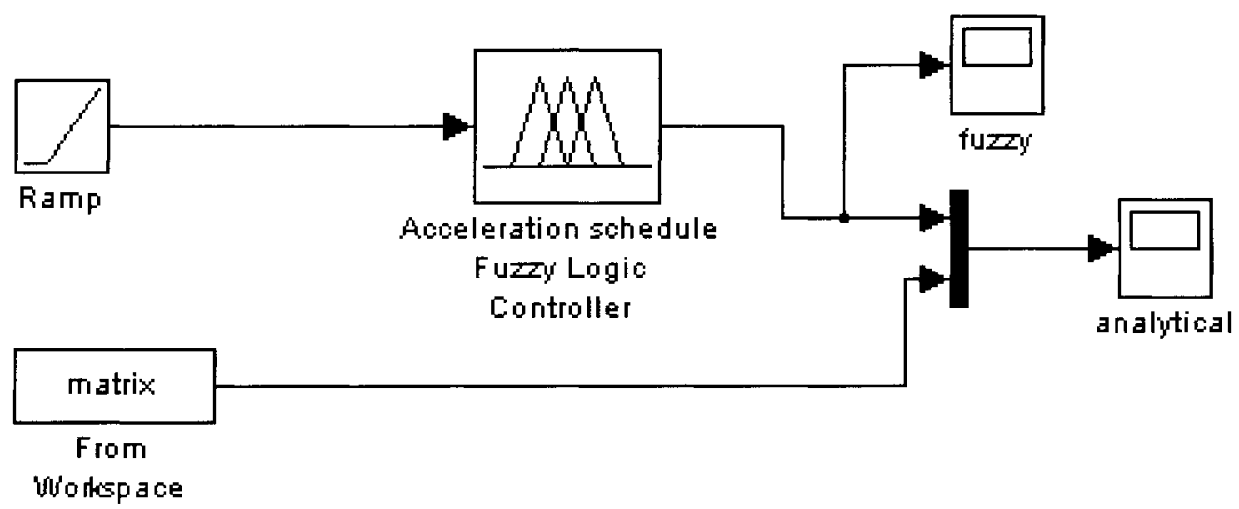

(a)

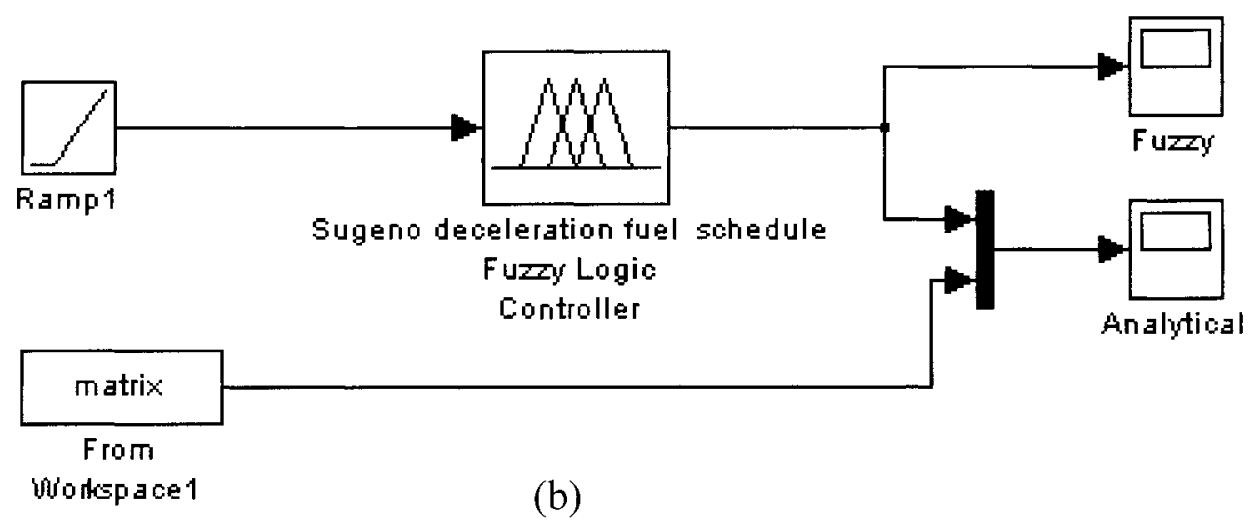

Figure C-1: Fuzzy fuel schedules for a 1 MW GTE: (a) Mamdani acceleration schedule, (b) Sugeno deceleration schedule 


\section{Acceleration deceleration Simulink model}

$\%$ Plots of the acceleration and deceleration schedules

$\%$ Acceleration schedule

schedule = ddeinit('excel','H:\TIT.xls');

speed $=$ ddereq(schedule, 'r18c14:r27c14');

fuel $=$ ddereq $($ schedule, 'r18c15:r27c15');

speed $=$ linspace $(0,650,10)^{\prime}$

matrix $=$ [speed, fuel $]$

plot(speed, fuel);

$\%$ Deceleration schedule

schedule = ddeinit('excel','H:\TIT.xls');

speed $=$ ddereq $($ schedule, 'r18c14:r27c14');

fuel $=$ ddereq $($ schedule, 'r18c16:r27c16');

speed $=$ linspace $(0,650,10)^{\prime}$

matrix $=$ [speed, fuel $]$

plot(speed, fuel);

\section{Sugeno deceleration fuel schedule Matlab codes}

\%rule base, inference engine,

[System]

Name $=$ 'sugenoDecc'

Type $=$ 'sugeno'

Version $=2.0$

NumInputs $=1$

NumOutputs $=1$

NumRules $=6$

AndMethod='prod'

OrMethod='probor'

ImpMethod='prod'

AggMethod='sum'

DefuzzMethod='wtaver'

\%input membership functions $\left(\mathrm{N}_{\mathrm{GG}}\right)$

[Input1]

Name $={ }^{\prime} \mathrm{N}_{\mathrm{GG}}{ }^{\prime}$

Range $=\left[\begin{array}{lll}2169028000\end{array}\right]$

NumMFs $=6$ 
MF1='NB':'trimf',[2170022320 23000]

MF2='N':'trimf,,[22400 2310023900$]$

MF3='NS':'trimf',[23500 24202 25000]

MF4='Zero':'trimf',[24500 25300 26122]

MF5='PS':'trimf',[25500 26272 27000]

MF6='P':'trimf',[26500 27257 28000]

$\%$ output membership functions $\left(\mathrm{Wf} / \mathrm{P}_{3}\right)$

[Output1]

Name $={ }^{\prime} \mathrm{Wf} / \mathrm{P}_{3}{ }^{\prime}$

Range $=\left[\begin{array}{ll}0 & 1\end{array}\right]$

NumMFs $=6$

MF1='NB':'constant',[7.2588]

$\mathrm{MF} 2={ }^{\prime} \mathrm{N}^{\prime}:$ 'constant',[7.408]

MF3='NS':'constant',[7.612]

MF4='Zero':'constant',[7.835]

MF5='PS':'constant',[8.085]

MF6='P':'constant',[9.231]

\%rules

[Rules]

$1,1(1): 1$

$2,2(1): 1$

$3,3(1): 1$

$4,4(1): 1$

$5,5(1): 1$

$6,6(1): 1$

\section{Mamdani acceleration fuel schedule Matlab codes}

\%rule base, inference engine,

[System]

Name $=$ 'FuelscheduleAcc'

Type='mamdani'

Version $=2.0$

NumInputs $=1$

NumOutputs $=1$

NumRules $=9$

AndMethod='min'

OrMethod='max'

ImpMethod=' $\mathrm{min}$ ' 


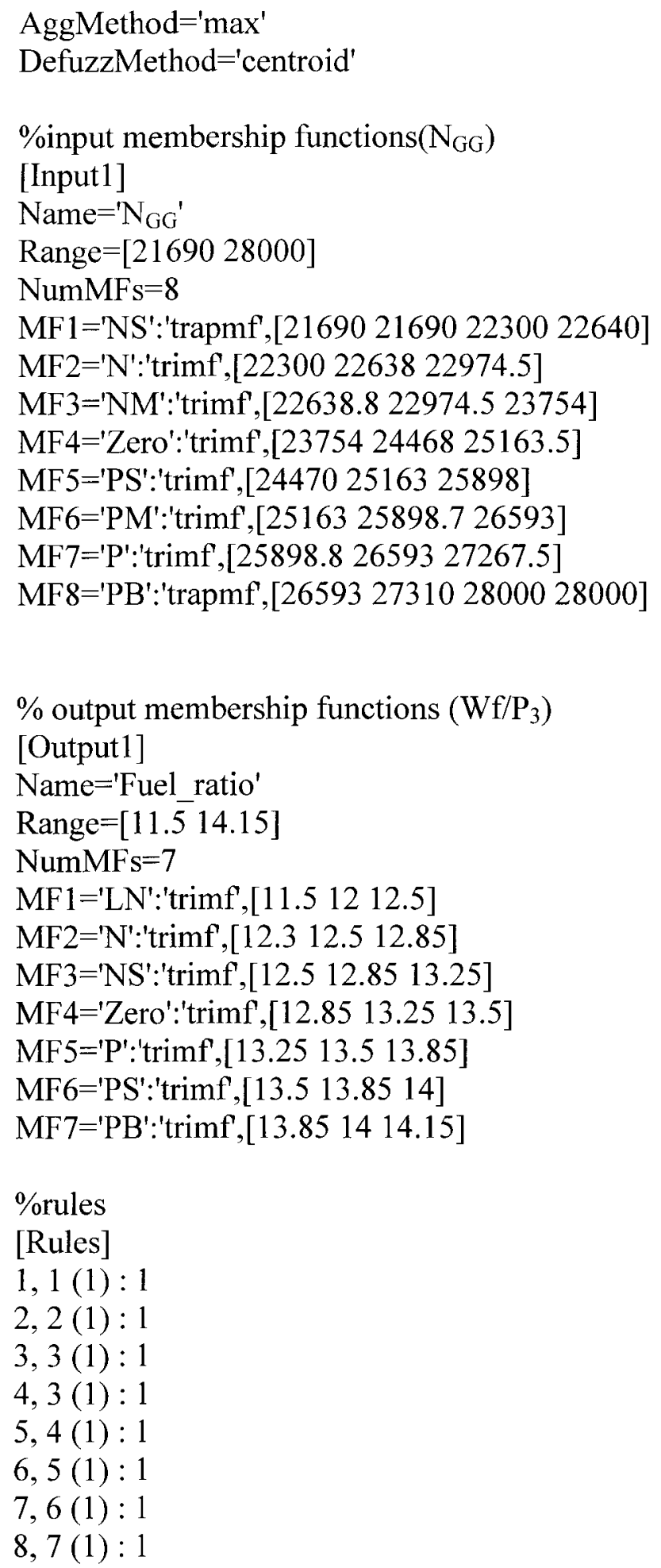




\section{Appendix D}

\section{Zero-Emission GT Model}

D-1 


\section{Description of the Zero-Emission GT model}

The model included in this appendix consists of a $60 \mathrm{~kW}$ single shaft gas turbine engine designed at Carleton University. The GTE is composed of a compressor with bleed control, a recuperator, a combustor and a turbine as shown in the Simulink model in D-3. The GTE intended application is to drive a power generator at a rated speed of 110000 rpm. It is controlled by a PID as shown in D-4. A fuzzy controller is designed in this thesis to replace the PID. The Matlab codes of the FLC are shown in D-5.

The purpose of the controller is to maintain a constant operating speed set at $110000 \mathrm{rpm}$ as the load varies. This is achieved by varying the fuel flow (mf) to the combustor. The controller compares the actual gas turbine speed (NA) with the set speed and passes the difference to the controller and accordingly produces a fuel flow output. The actual speed and compressor discharge pressure (P03) are passed through the fuel schedule and a limiting fuel flow value is produced. The two fuel flow outputs (from the controller and the fuel schedule) are compared on a minimum basis. The lower value for a particular load is chosen to avoid surge or over-temperature. This value is denoted as the required fuel flow to be added to the combustor $(\mathrm{mf})$.

The Simulimk model of the GTE and the fuzzy controller along with the Matlab codes are included as follows: 


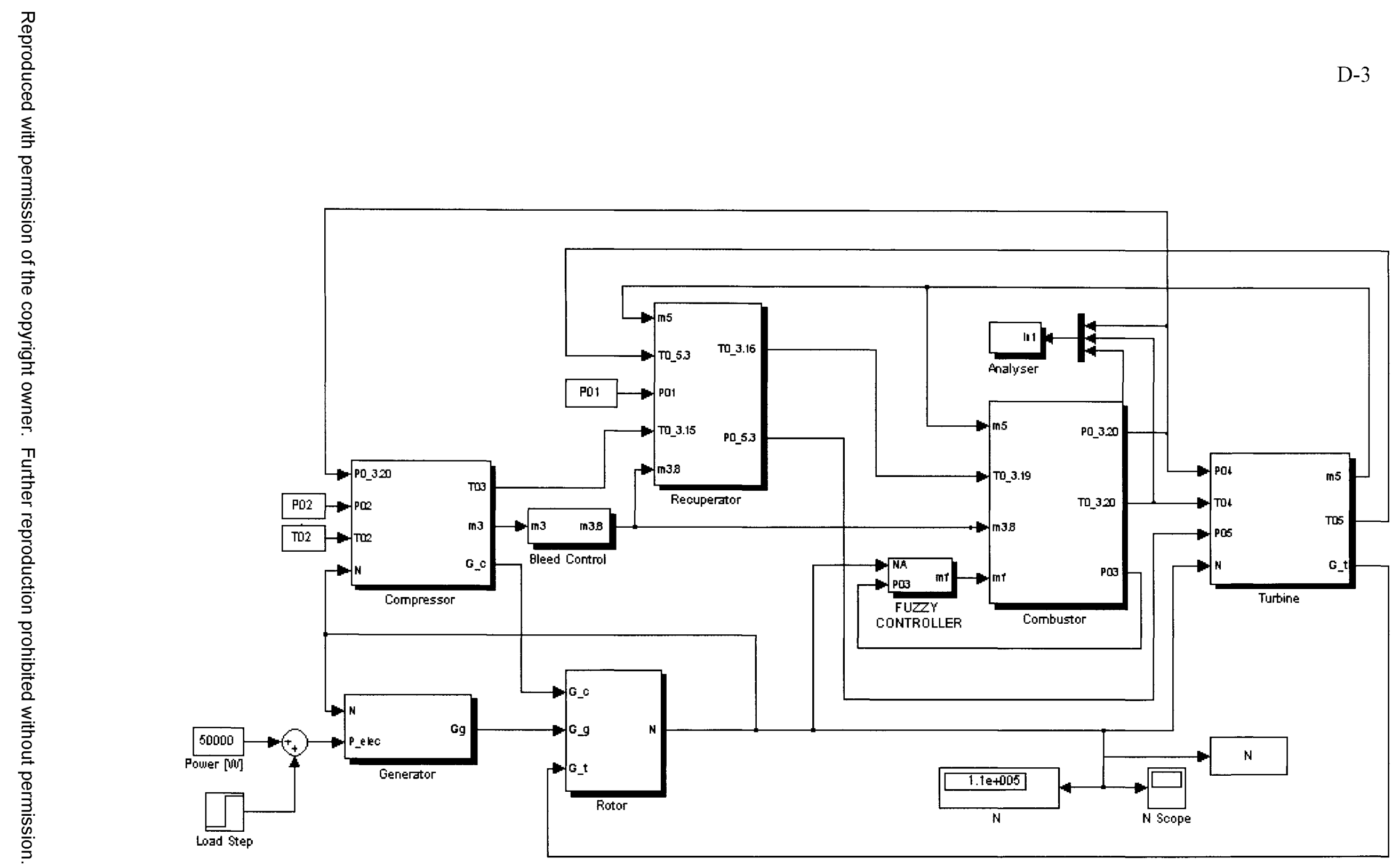

Figure D-1: Zero-Emission GT Simulink model 
PID

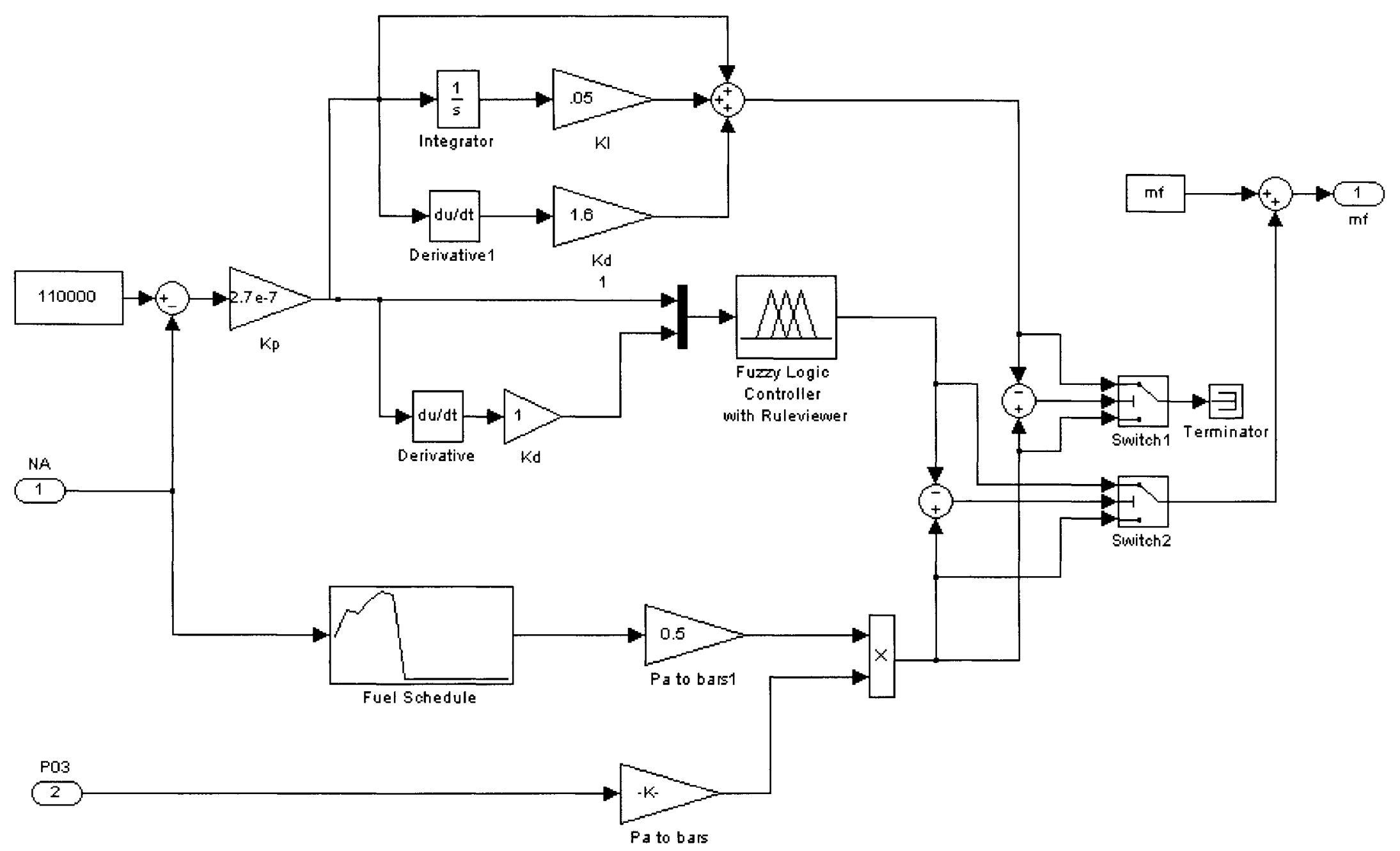

Figure D-2: Fuzzy control configuration of Zero-Emission GT 


\section{Sugeno FLC for $60 \mathrm{~kW}$ gas turbine engine Matlab codes}

$\%$ Inference engine (Sugeno)

$\%$ the two inputs to the system are the speed error (Eerrorspeed)

$\%$ and the rate of speed of the speed error (Eerrordot)

[System]

Name='speedDPzip'

Type $=$ 'sugeno'

Version $=2.0$

NumInputs $=2$

NumOutputs $=1$

NumRules $=16$

AndMethod='prod'

OrMethod='probor'

ImpMethod='prod'

AggMethod='sum'

DefuzzMethod='wtaver'

$\%$ Input membership functions (Errorspeed variable)

[Input1]

Name $=$ 'errorspeed'

Range $=\left[\begin{array}{ll}-0.01 & 0.007\end{array}\right]$

NumMFs $=4$

MF1='NB':'trimf',[-0.0102 $-0.007818-0.00499]$

MF2='N':'trimf',[-0.00708 $-0.0029160 .000456]$

MF3='NS':'trimf',[-0.002232 0.00050120 .003025$]$

MF4='Zero':'trapmf',[0.001758 0.003064 0.007 0.007]

$\%$ Input membership functions (errordot variable)

[Input2]

Name $=$ 'errordot'

Range $=[-0.00134 \mathrm{e}-005]$

NumMFs $=4$

$\mathrm{MF} 1=$ ='NB':'trapmf',[-0.00178 $-0.00135-0.001195-0.00107]$

MF2='N':'trimf',[-0.00117-0.000901 -0.000649]

MF3='NS':'trapmf',[-0.000763 $-0.000628-0.0005041-0.000338]$

MF4='Zero':'trapmf',[-0.0005116 -0.0002881 .0498 2.5]

$\%$ Output membership functions (fuel flow)

[Output1]

Name $=$ 'fuel'

Range $=\left[\begin{array}{ll}0 & 1\end{array}\right]$

NumMFs $=4$

MF1='NB':'constant', $[-0.00032]$ 
MF2='NS':'constant',[-0.00038]

MF3='P':'constant', [-0.000393]

MF4='PB':'constant',[-0.007]

$\%$ rule-base matrix

[Rules]

$11,1(1): 1$

$12,3(1): 1$

$13,2(1): 1$

$14,4(1): 1$

$21,4(1): 1$

$22,3(1): 1$

24,2 (1): 1

2 3, 4 (1): 1

$31,3(1): 1$

$32,1(1): 1$

33,4 (1): 1

34,3 (1): 1

$41,1(1): 1$

$42,3(1): 1$

43,2 (1): 1

4 4, $1(1): 1$

\section{Modified membership functions FLC for $60 \mathrm{~kW}$ GTE}

[System]

Name $=$ 'speedDPfinal'

Type $=$ 'sugeno'

Version $=2.0$

NumInputs $=2$

NumOutputs $=1$

NumRules $=16$

AndMethod='prod'

OrMethod='probor'

ImpMethod='prod'

AggMethod='sum'

DefuzzMethod='wtaver'

[Input 1]

Name $=$ 'errorspeed'

Range $=\left[\begin{array}{ll}-0.01 & 0.007\end{array}\right]$

NumMFs $=4$

MF1='NB':'trapmf',[-0.01003 -0.01003 -0.006501 -0.003855]

MF2='N':'trimf',[-0.007168 $-0.0029180 .001332]$ 
MF3='NS':'trimf',[-0.002232 0.00050120 .003025$]$

MF4='Zero':'trapmf',[0.001758 0.003064 0.007 0.007]

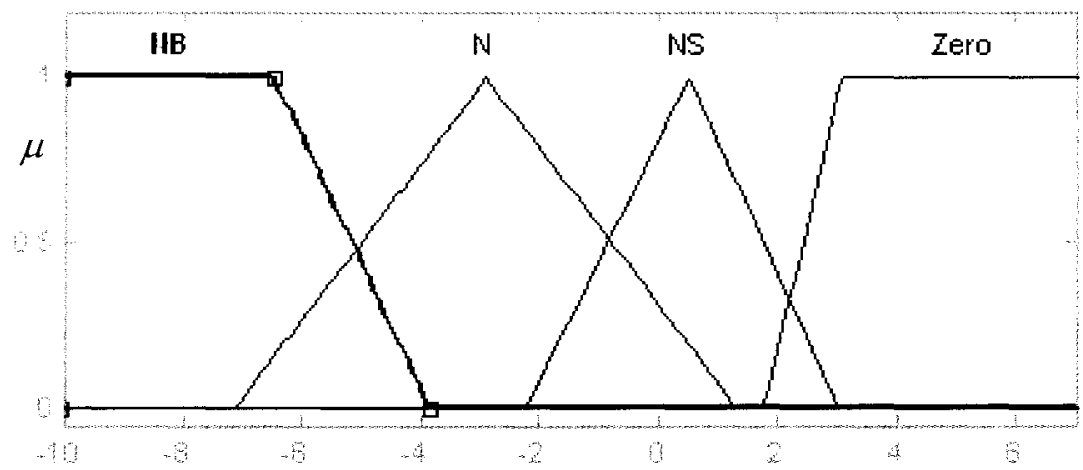

Figure D-3: FLC Errorspeed input modified membership functions; The "NB" is changed to trapezoidal function

[Input2]

Name ${ }^{\prime}$ 'errordot'

Range $=[-0.00134 \mathrm{e}-005]$

NumMFs $=4$

$\mathrm{MF} 1={ }^{\prime} \mathrm{NB}$ ':'trapmf',[-0.001782 -0.001354 -0.001245 -0.001133]

MF2='N':'trimf',[-0.00122 $-0.001064-0.0008852]$

MF3='NS':'trimf',[-0.001026 $-0.0007771-0.0005502]$

MF4='Zero':'trapmf',[-0.0007102 $-0.00048691 .052 .5]$

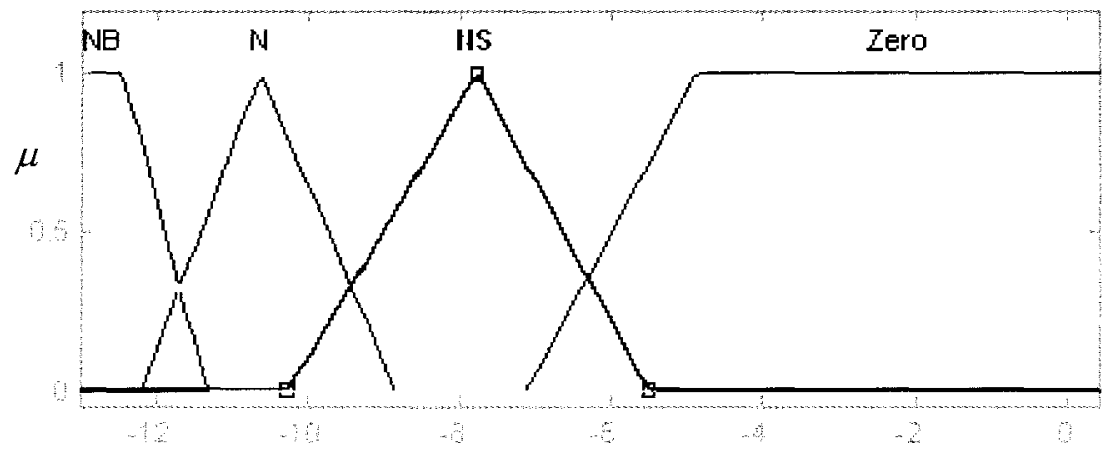

Figure D-4: FLC Errordot input modified membership functions;

The "NS" is changed to triangular function and the "N", "NS", and "Zero" memberships are adjusted

$\%$ Output membership function (fuel flow) is as per Figure 5-16.

[Output1]

Name $=$ 'fuel'

Range $=\left[\begin{array}{ll}0 & 1\end{array}\right]$

NumMFs $=4$ 
MF1='NB':'constant',[-0.00032]

MF2='NS':'constant',[-0.00038]

MF3='P':'constant',[-0.000393]

MF4='PB':'constant',[-0.007]

[Rules]

$11,1(1): 1$

$12,3(1): 1$

$13,2(1): 1$

$14,4(1): 1$

$21,4(1): 1$

$22,3(1): 1$

$23,4(1): 1$

$24,2(1): 1$

$31,3(1): 1$

$32,1(1): 1$

$33,4(1): 1$

$34,3(1): 1$

$41,1(1): 1$

$42,3(1): 1$

4 3, 2 (1): 1

4 4, 1 (1): 1 


\section{Appendix E}

\section{Artificial Neural Networks Codes}

E-1 
ANNs for the TIT versus fuel flow and $\mathrm{N}_{\mathrm{GG}}$ versus fuel flow of a $1 \mathrm{MW}$ GTE (at transient performance).

The transient performance evaluation of GTEs is an important factor in determining their response to varying fuel flows. This factor is crucial in the design phase of the GTEs. In this appendix, ANNs are used to model the transient performance of a $1 \mathrm{MW}$ twin shaft. This is done by duplicating the relationships, built in GasTurb simulation program, between the parameters defining the model. These are the TIT, $\mathrm{N}_{\mathrm{GG}}$, and fuel flow. For this purpose two two-layer feedforward artificial neural networks were built. One relating the TIT to fuel flow (neural network TIT) and the second identifying the relashionship between $\mathrm{N}_{\mathrm{GG}}$ and fuel flow (neural network $\mathrm{N}_{\mathrm{GG}}$ ). The simulink model and Matlab codes for the two ANNs are included hereafter. The "validspeed" and "testspeed" blocks shown represent the validation and test data for the neural network $\mathrm{N}_{\mathrm{GG}}$. The "validTIT" and "testTIT" blocks shown embed the validation and test data for the neural network TIT. These data are generated by GasTurb program and are read from excel file. 


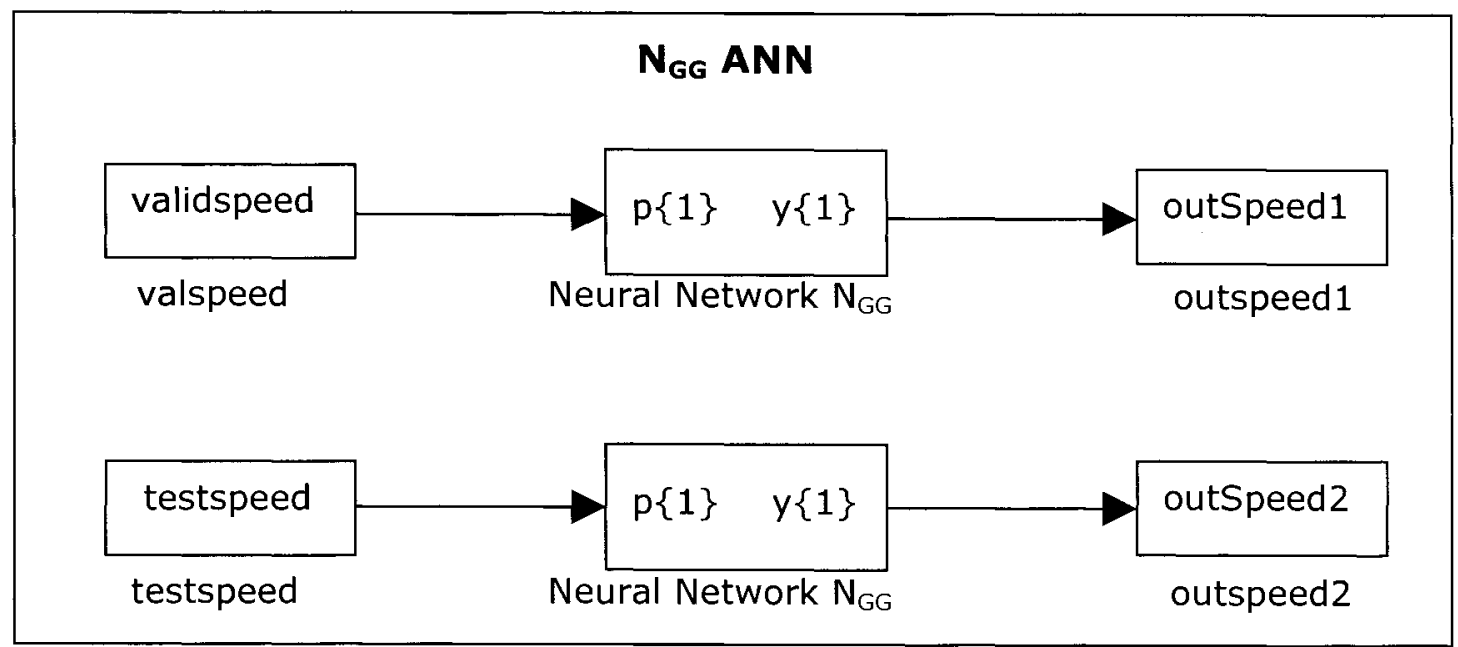

(a)

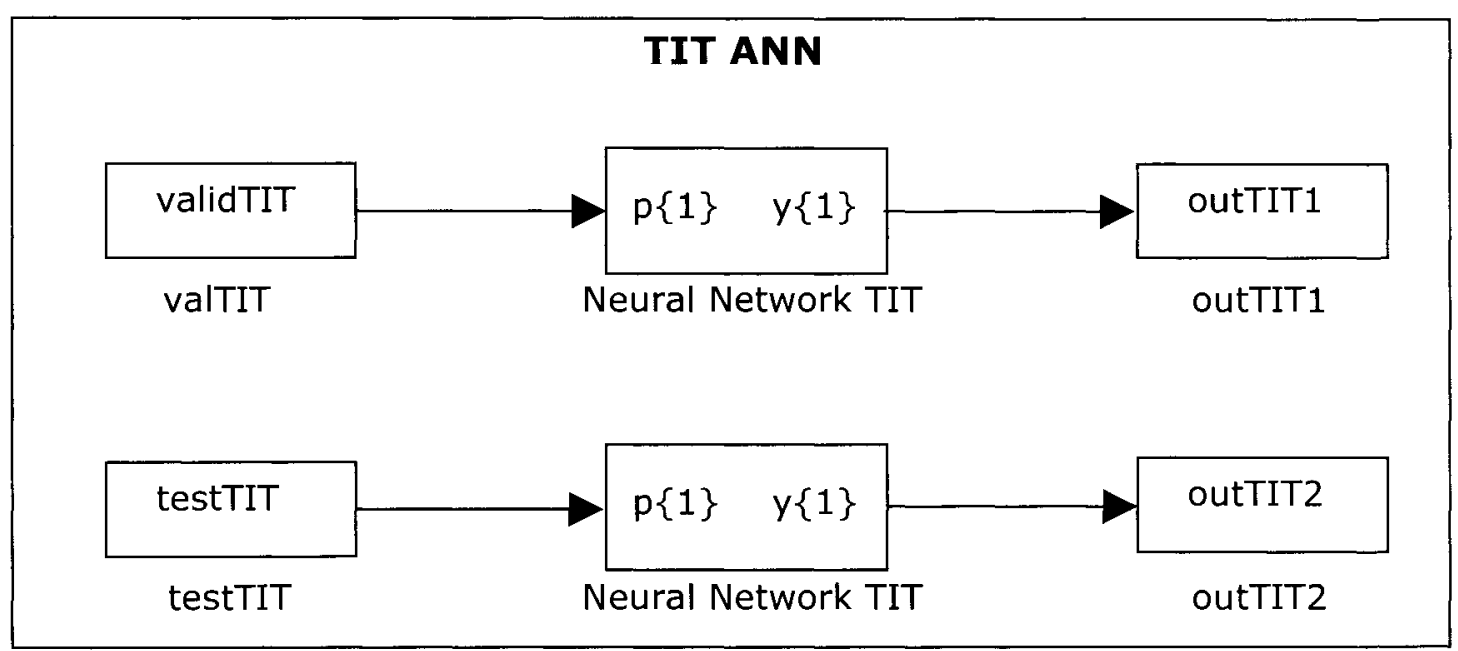

(b)

Figure E-1: ANN Simulink models for a $1 \mathrm{MW}$ GTE; (a) $\mathrm{N}_{\mathrm{GG}}$ ANN, (b) TIT ANN 


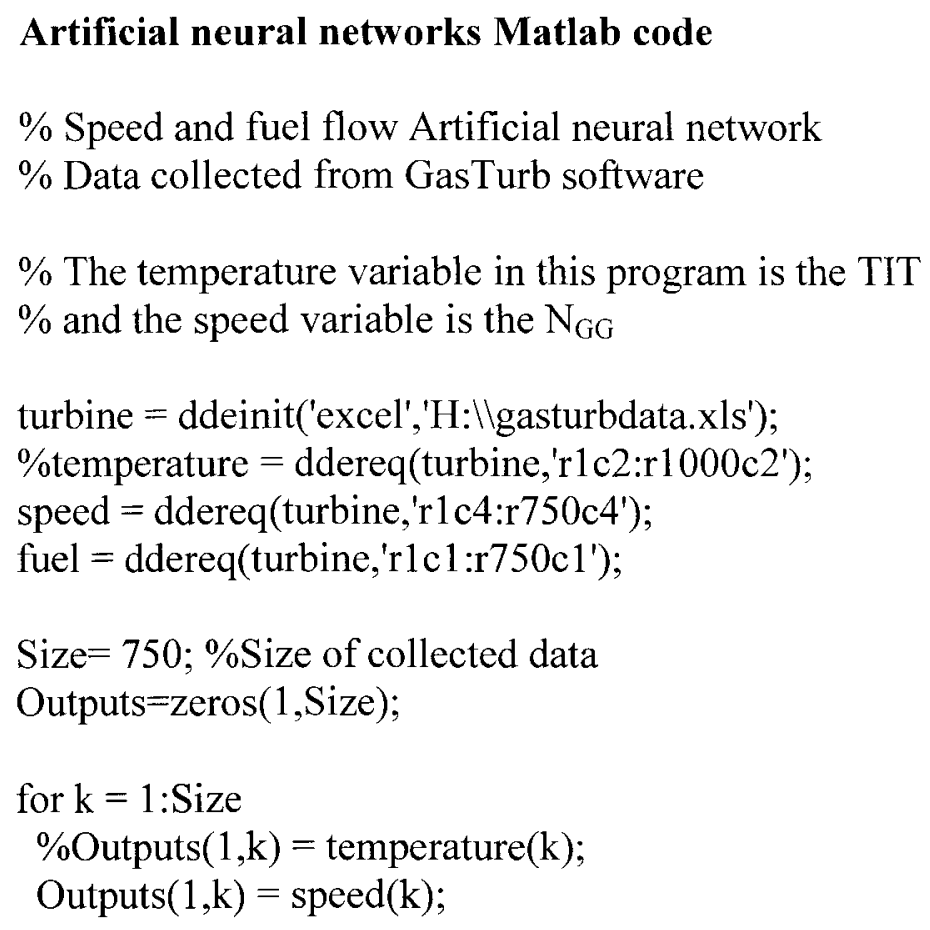

$\%$ TIT and fuel flow Artificial neural network $\%$ data collected from GasTurb software

turbine = ddeinit('excel','H:||gasturbdata.xls'); temperature $=$ ddereq $\left(\right.$ turbine, ${ }^{\prime}$ 1 $1 \mathrm{c} 2:$ r706 $\left.22^{\prime}\right)$; 
fuel $=$ ddereq(turbine,'r1 $1:$ r706c1');

Size $=706 ; \%$ Size of collected data

Outputs=zeros $(1$, Size $)$;

for $\mathrm{k}=1$ :Size

$\operatorname{Outputs}(1, \mathrm{k})=$ temperature $(\mathrm{k})$;

$\%$ Outputs $(1, \mathrm{k})=\operatorname{speed}(\mathrm{k})$;

end

\%Artificial neural network generation

net=newff(minmax(Outputs),[9, 1],\{'tansig','purelin'\},'trainlm');

net.trainParam. show $=100$;

net.trainParam.1r $=0.005$;

net.trainParam.lr_inc $=1.05$;

net.trainParam.epochs $=3000$;

net.trainParam.goal $=1 \mathrm{e}-10$;

net $=\operatorname{train}($ net, Outputs( $(1: 1,1:$ Size $)$, fuel'); $\%$ fuel=Output

\%Simulink model

gensim(net,1);

validTIT=xlsread(gasturbdata,fl:g165);

testTIT $=$ xlsread (gasturbdata,h1:i78);

$\%$ to workspace outTIT

$\%$ to excel spreadsheet

xlswrite(outTIT1,'sheet1')

xlswrite(outTIT2,'sheet2')

$\%$ Comparing the ANNs are done through Simulink

$\%$ files copied to Excel spreadsheets. The performance is evaluated

$\%$ and the ANN graphs are built. 